\title{
Direct and Oxidant-Free Electron-Deficient Arylation of
}

\author{
$\mathrm{N}$-Acyl-Protected Tetrahydroisoquinolines \\ Changcun Yan, ${ }^{1}$ Lili Li, ${ }^{1}$ Yuxiu Liu, ${ }^{1}$ and Qingmin Wang* ${ }^{* 1,2}$ \\ ${ }^{1}$ State Key Laboratory of Elemento-Organic Chemistry, Research Institute of Elemento-Organic \\ Chemistry, Nankai University, Tianjin 300071, People's Republic of China \\ ${ }^{2}$ Collaborative Innovation Center of Chemical Science and Engineering (Tianjin), Tianjin 300071, \\ People's Republic of China \\ Supporting Information
}

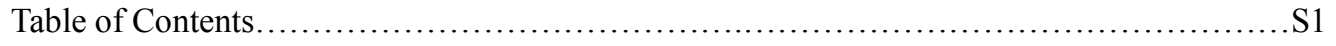

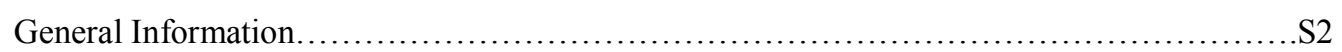

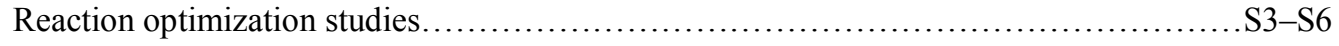

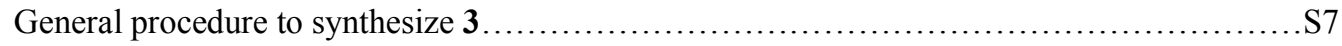

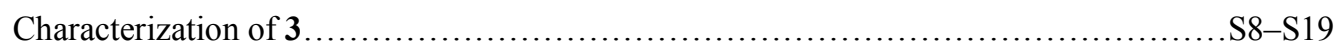

Procedure for the synthesis $\mathbf{3 a b}$ under sunlight on a gram scale $\ldots \ldots \ldots \ldots \ldots \ldots \ldots \ldots \ldots \ldots$ S20

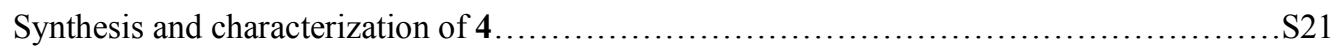

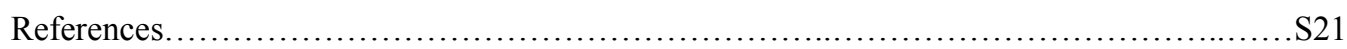

Copies of ${ }^{1} \mathrm{H}$ NMR and ${ }^{13} \mathrm{C}$ NMR spectra for new compounds........................S22-S42 
General Information: All reagents were used as received. Dimethylacetamide (DMA) was distilled from $\mathrm{CaH}_{2} . \mathrm{Ph}_{3} \mathrm{SiSH}$ was synthesized with a previously described procedure. ${ }^{\mathrm{S} 1}{ }^{1} \mathrm{H}$ and ${ }^{13} \mathrm{C}$ Nuclear Magnetic Resonance (NMR) spectra were recorded on Bruker Avance 400 Ultrashield NMR spectrometers. Chemical shifts $(\delta)$ were given in parts per million (ppm) and were measured downfield from internal tetramethylsilane. High-resolution mass spectrometry (HRMS) data were obtained on an FTICR-MS instrument (Ionspec 7.0 T). The melting points were determined on an X-4 microscope melting point apparatus and are uncorrected. Conversion was monitored by thin layer chromatography (TLC). Flash column chromatography was performed over silica gel (100-200 mesh). 


\section{Reaction optimization studies}

Table 1. Optimization of bases, solvents and HAT catalysts ${ }^{a}$

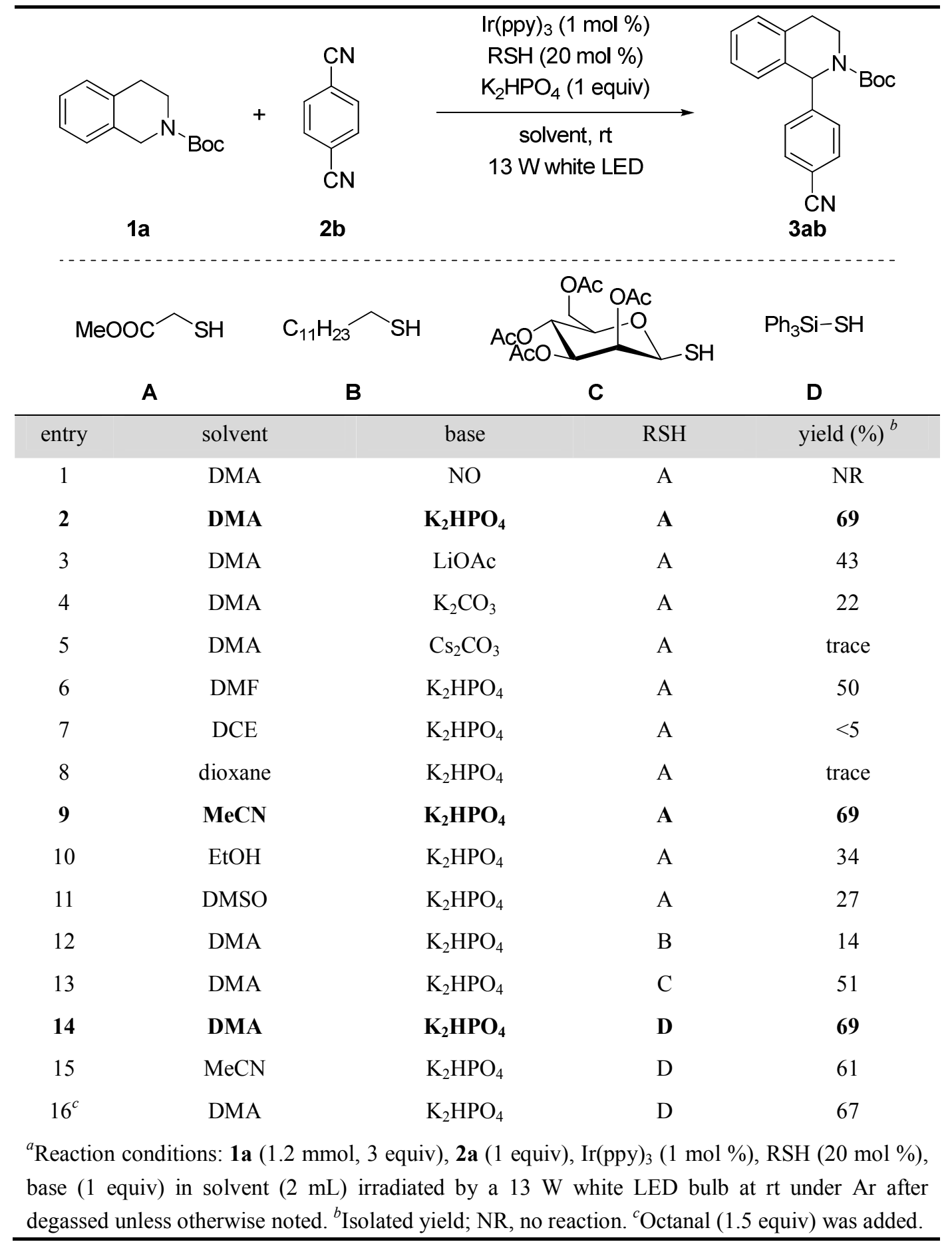


Table 2. Optimization of HAT catalysts ${ }^{a}$

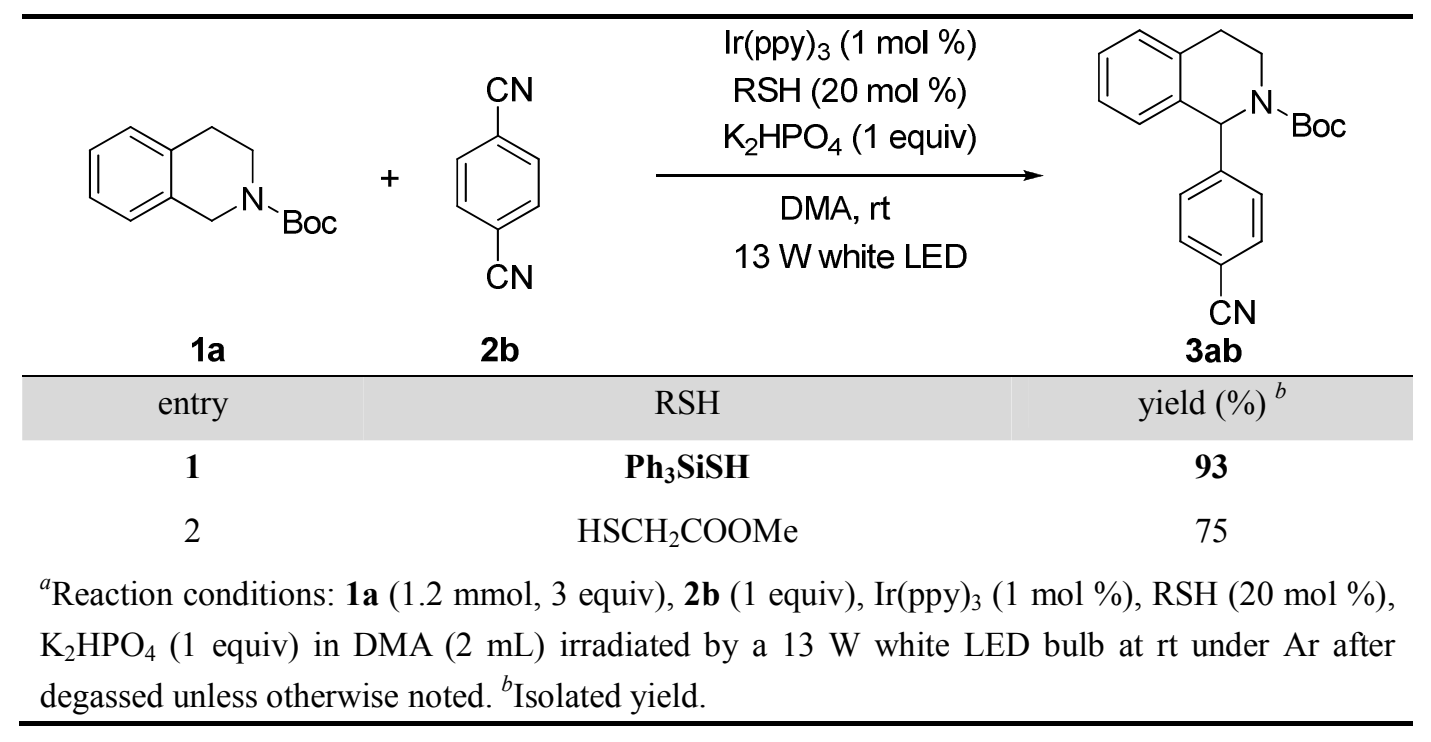


Table 3. Optimization of dosage of substrates and HAT catalysts ${ }^{a}$

\begin{tabular}{|c|c|c|c|c|}
\hline & & $\begin{array}{r}\operatorname{Ir}(\mathrm{p} \\
\mathrm{Ph}_{3} \mathrm{~S} \\
\mathrm{~K}_{2} \mathrm{H} \\
\\
13\end{array}$ & & $\begin{array}{l}\mathrm{CN} \\
3 \mathrm{ab}\end{array}$ \\
\hline entry & $1 \mathrm{a}$ & $2 b$ & $\mathrm{X}$ & yield $(\%)^{b}$ \\
\hline 1 & 1 equiv & 2 equiv & 20 & 66 \\
\hline 2 & 1 equiv & 1.5 equiv & 20 & 58 \\
\hline 3 & 1 equiv & 1.2 equiv & 20 & 61 \\
\hline 4 & 1 equiv & 1 equiv & 20 & 61 \\
\hline 5 & 1.2 equiv & 1 equiv & 20 & 73 \\
\hline 6 & 1.5 equiv & 1 equiv & 20 & 91 \\
\hline 7 & 2 equiv & 1 equiv & 20 & 94 \\
\hline 8 & 3 equiv & 1 equiv & 20 & 93 \\
\hline 9 & 2 equiv & 1 equiv & 10 & 94 \\
\hline 10 & 2 equiv & 1 equiv & 5 & 95 \\
\hline 11 & 2 equiv & 1 equiv & 2 & 81 \\
\hline 12 & 2 equiv & 1 equiv & 1 & 71 \\
\hline \multicolumn{5}{|c|}{$\begin{array}{l}{ }^{a} \text { Reaction conditions: } \mathbf{1 a}, 2 \mathbf{b}, \operatorname{Ir}(\mathrm{ppy})_{3}(1 \mathrm{~mol} \%), \mathrm{Ph}_{3} \mathrm{SiSH}(\mathrm{X} \mathrm{mol} \%), \mathrm{K}_{2} \mathrm{HPO}_{4}(1 \text { equiv) in DMA } \\
(2 \mathrm{~mL}) \text { irradiated by a } 13 \mathrm{~W} \text { white } \mathrm{LED} \text { bulb at } \mathrm{rt} \text { under } \mathrm{Ar} \text { after degassed unless otherwise noted. } \\
{ }^{b} \text { Isolated yield. }\end{array}$} \\
\hline
\end{tabular}


Table 4. Control experiments ${ }^{a}$

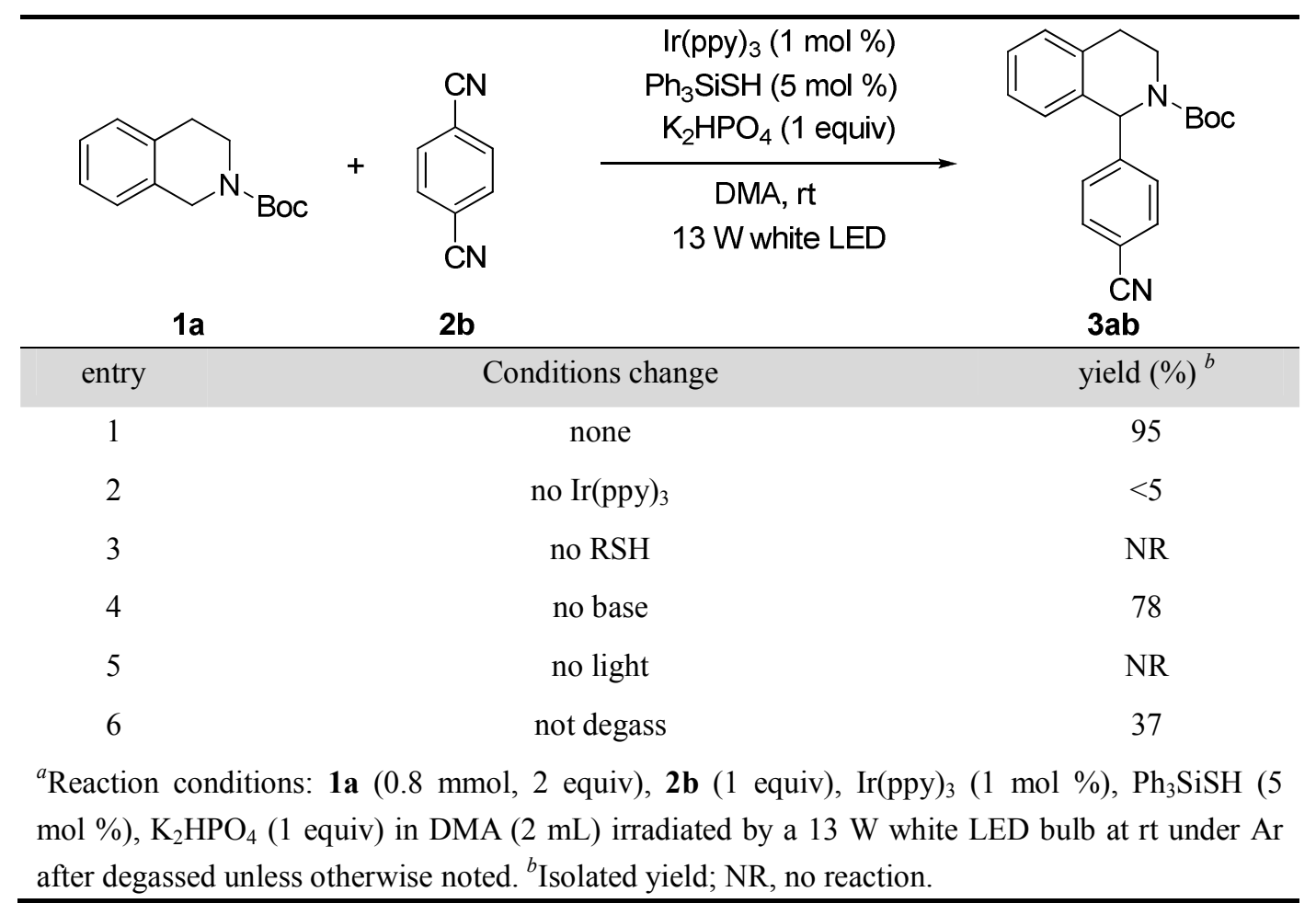




\section{General procedure for the synthesis of 3.}

An oven dried Schlenk tube was charged with substrate 1 ( $0.8 \mathrm{mmol}, 2$ equiv), 2 ( $0.4 \mathrm{mmol}, 1$ equiv), $\mathrm{K}_{2} \mathrm{HPO}_{4}$ (0.4 mmol, 1 equiv), $\mathrm{Ph}_{3} \mathrm{SiSH}(0.02 \mathrm{mmol}, 5 \mathrm{~mol} \%), \operatorname{Ir}(\mathrm{ppy})_{3}(4 \mu \mathrm{mol}, 1 \mathrm{~mol} \%)$ and DMA $(2 \mathrm{~mL})$. The tube was evacuated and backfilled with argon (this process was repeated three times). The reaction mixture was stirred under the irradiation with a $13 \mathrm{~W}$ white LED bulb (commercially available Philips' LED bulb) at room temperature for 2-24 h until a presistent green/yellow fluorescence was generally observed. The reaction mixture was diluted with water, extracted with EtOAc ( $3 \times 5 \mathrm{~mL})$, combined organic layers were washed with brine, dried by anhydrous $\mathrm{Na}_{2} \mathrm{SO}_{4}$, and concentrated in vacuo. The crude product was purified by common column chromatography using the indicated solvent system to give the desired compounds.

\section{(Caution: cyanide generated during the course of the reaction)}

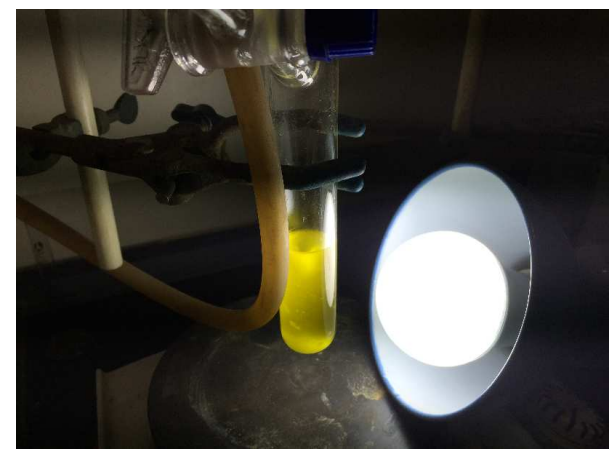

Irradiation with a $13 \mathrm{~W}$ white LED bulb

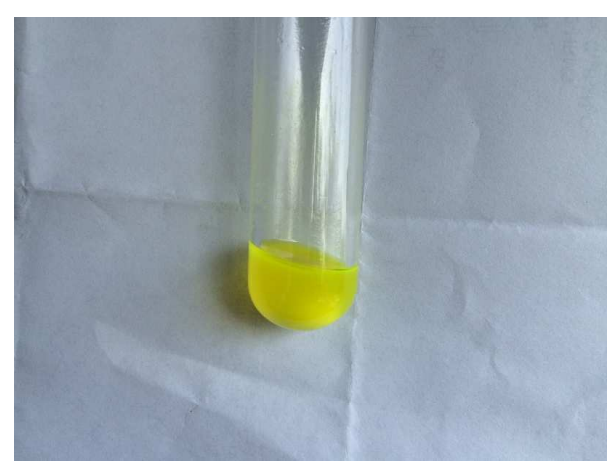

Presistent green/yellow fluorescence 


\section{Characterization of 3}

tert-Butyl 1-(4-(methoxycarbonyl)phenyl)-3,4-dihydroisoquinoline-2(1H)-carboxylate (3aa).<smiles>COC(=O)c1ccc(C2c3ccccc3CCN2C(=O)OC(C)(C)C)cc1</smiles>

Prepared according to the general method and purified by common column chromatography $(\mathrm{PE}: \mathrm{EA}=20: 1)$ to give the title compounds as a colorless viscous oil (78 mg, yield: 53\%).

${ }^{1} \mathrm{H}$ NMR (400 MHz, $\mathrm{CDCl}_{3}$, rotamers seen) $\delta 7.95(\mathrm{~d}, J=8.0 \mathrm{~Hz}, 2 \mathrm{H}), 7.30(\mathrm{~d}, J=8.0 \mathrm{~Hz}, 2 \mathrm{H})$, $7.26-7.15(\mathrm{~m}, 3 \mathrm{H}), 7.11-6.99(\mathrm{~m}, 1 \mathrm{H}), 6.59-6.07(\mathrm{~m}, 1 \mathrm{H}), 4.17-3.74(\mathrm{~m}, 4 \mathrm{H}), 3.30-3.17(\mathrm{~m}$, 1H), $3.05-2.87(\mathrm{~m}, 1 \mathrm{H}), 2.85-2.67(\mathrm{~m}, 1 \mathrm{H}), 1.48(\mathrm{~s}, 9 \mathrm{H})$.

${ }^{13} \mathrm{C}$ NMR $\left(100 \mathrm{MHz}, \mathrm{CDCl}_{3}\right.$, rotamers seen) $\delta 166.9,154.6,148.2,135.3,135.0,129.6,129.1$, $129.0,128.1,127.2,126.3,80.3,57.4,52.1,38.6,28.5$.

HRMS (ESI) calcd for $\mathrm{C}_{22} \mathrm{H}_{25} \mathrm{NNaO}_{4}[\mathrm{M}+\mathrm{Na}]^{+}$390.1681, found 390.1671.

tert-Butyl 1-(4-cyanophenyl)-3,4-dihydroisoquinoline-2(1H)-carboxylate (3ab).<smiles>CC(=O)OC(=O)N1CCc2ccccc2C1c1ccc(C#N)cc1</smiles>

Prepared according to the general method and purified by common column chromatography $(\mathrm{PE}: \mathrm{EA}=20: 1)$ to give the title compounds as a colorless viscous oil (127 mg, yield: 95\%).

${ }^{1} \mathrm{H}$ NMR (400 MHz, $\mathrm{CDCl}_{3}$, rotamers seen) $\delta 7.57(\mathrm{~d}, J=8.0 \mathrm{~Hz}, 2 \mathrm{H}), 7.35(\mathrm{~d}, J=8.0 \mathrm{~Hz}, 2 \mathrm{H})$, $7.28-7.13(\mathrm{~m}, 3 \mathrm{H}), 7.11-6.92(\mathrm{~m}, 1 \mathrm{H}), 6.58-6.05(\mathrm{~m}, 1 \mathrm{H}), 4.20-3.80(\mathrm{~m}, 1 \mathrm{H}), 3.27-3.14(\mathrm{~m}$, 
$1 \mathrm{H}), 3.03-2.86(\mathrm{~m}, 1 \mathrm{H}), 2.83-2.64(\mathrm{~m}, 1 \mathrm{H}), 1.48(\mathrm{~s}, 9 \mathrm{H})$.

${ }^{13} \mathrm{C}$ NMR (100 MHz, $\mathrm{CDCl}_{3}$, rotamers seen) $\delta 154.7,148.5,135.3,135.0,134.4,132.1,129.1$, $128.8,128.3,127.9,127.5,126.4,118.8,111.1,80.6,57.3,38.9,28.5$.

HRMS (ESI) calcd for $\mathrm{C}_{21} \mathrm{H}_{22} \mathrm{NNaO}_{2}[\mathrm{M}+\mathrm{Na}]^{+} 357.1579$, found 357.1576.

tert-Butyl 1-(4-(methylsulfonyl)phenyl)-3,4-dihydroisoquinoline-2(1H)-carboxylate (3ac).<smiles>CC(=O)OC(C)(C)OC(=O)N1CCc2ccccc2C1c1ccc(S(C)(=O)=O)cc1</smiles>

Prepared according to the general method and purified by common column chromatography $(\mathrm{PE}: \mathrm{EA}=5: 1)$ to give the title compounds as a colorless viscous oil (97 $\mathrm{mg}$, yield: $63 \%$ ).

${ }^{1} \mathrm{H}$ NMR (400 MHz, $\mathrm{CDCl}_{3}$, rotamers seen) $\delta 7.85(\mathrm{~d}, J=8.0 \mathrm{~Hz}, 2 \mathrm{H}), 7.44(\mathrm{~d}, J=8.0 \mathrm{~Hz}, 2 \mathrm{H})$, $7.32-7.16(\mathrm{~m}, 3 \mathrm{H}), 7.13-6.96(\mathrm{~m}, 1 \mathrm{H}), 6.58-6.13(\mathrm{~m}, 1 \mathrm{H}), 4.19-3.82(\mathrm{~m}, 1 \mathrm{H}), 3.30-3.18(\mathrm{~m}$, 1H), 3.04 (s, 3H), $3.03-2.89(\mathrm{~m}, 1 \mathrm{H}), 2.85-2.68(\mathrm{~m}, 1 \mathrm{H}), 1.49(\mathrm{~s}, 9 \mathrm{H})$.

${ }^{13} \mathrm{C}$ NMR (100 MHz, $\mathrm{CDCl}_{3}$, rotamers seen) $\delta 149.4,139.3,135.3,134.5,129.0,128.3,127.5$, $127.4,126.4,80.5,57.3,44.5,38.9,28.5$.

HRMS (ESI) calcd for $\mathrm{C}_{21} \mathrm{H}_{26} \mathrm{NO}_{4} \mathrm{~S}[\mathrm{M}+\mathrm{H}]^{+} 388.1583$, found 388.1573 .

tert-Butyl

1-(1-oxo-1,3-dihydroisobenzofuran-5-yl)-3,4-dihydroisoquinoline-2(1H)-carboxylate (3ad).<smiles>CC(C)(C)OC(=O)N1CCc2ccccc2C1c1ccc2c(c1)COC2=O</smiles> 
Prepared according to the general method and purified by common column chromatography $(\mathrm{PE}: \mathrm{EA}=5: 1)$ to give the title compounds as a colorless viscous oil $(55 \mathrm{mg}$, yield: $38 \%$ ).

${ }^{1} \mathrm{H}$ NMR $\left(400 \mathrm{MHz}, \mathrm{CDCl}_{3}\right.$, rotamers seen) $\delta 7.84(\mathrm{~d}, J=8.0 \mathrm{~Hz}, 1 \mathrm{H}), 7.45(\mathrm{~d}, J=6.4 \mathrm{~Hz}, 1 \mathrm{H})$, $7.33(\mathrm{~s}, 1 \mathrm{H}), 7.28-7.16(\mathrm{~m}, 4 \mathrm{H}), 7.13-6.97(\mathrm{~m}, 1 \mathrm{H}), 6.66-6.12(\mathrm{~m}, 1 \mathrm{H}), 5.26(\mathrm{~s}, 2 \mathrm{H}), 4.32-$ $3.84(\mathrm{~m}, 1 \mathrm{H}), 3.31-3.18(\mathrm{~m}, 1 \mathrm{H}), 3.06-2.90(\mathrm{~m}, 1 \mathrm{H}), 2.86-2.70(\mathrm{~m}, 1 \mathrm{H}), 1.49(\mathrm{~s}, 9 \mathrm{H})$.

${ }^{13} \mathrm{C}$ NMR (100 MHz, $\mathrm{CDCl}_{3}$, rotamers seen) $\delta 170.8,154.9,150.2,146.9,135.2,134.6,129.3$ $129.1,128.4,127.5,126.4,125.6,124.7,121.7,80.6,69.6,57.6,38.7,28.5$.

HRMS (ESI) calcd for $\mathrm{C}_{22} \mathrm{H}_{24} \mathrm{NO}_{4}[\mathrm{M}+\mathrm{H}]^{+} 366.1705$, found 366.1701 .

tert-Butyl 1-(2,5-dichloro-4-cyanophenyl)-3,4-dihydroisoquinoline-2(1H)-carboxylate (3ae).<smiles>CC(C)(C)OC(=O)N1CCc2ccccc2C1c1cc(Cl)c(C#N)cc1Cl</smiles>

Prepared according to the general method and purified by common column chromatography $(\mathrm{PE}: \mathrm{EA}=10: 1)$ to give the title compounds as a white solid $(138 \mathrm{mg}$, yield: $86 \%)$, m.p. $=$ $120-121{ }^{\circ} \mathrm{C}$.

${ }^{1} \mathrm{H}$ NMR $\left(400 \mathrm{MHz}, \mathrm{CDCl}_{3}\right) \delta 7.72(\mathrm{~d}, J=1.6 \mathrm{~Hz}, 1 \mathrm{H}), 7.44(\mathrm{~d}, J=7.6 \mathrm{~Hz}, 1 \mathrm{H}), 7.24-7.18(\mathrm{~m}$, 2H), $7.17-7.10(\mathrm{~m}, 1 \mathrm{H}), 7.02(\mathrm{~d}, J=7.6 \mathrm{~Hz}, 1 \mathrm{H}), 4.30-3.98(\mathrm{~m}, 1 \mathrm{H}), 3.63-3.26(\mathrm{~m}, 1 \mathrm{H}), 3.09$ $-2.81(\mathrm{~m}, 2 \mathrm{H}), 1.36(\mathrm{~s}, 9 \mathrm{H})$.

${ }^{13} \mathrm{C}$ NMR $\left(100 \mathrm{MHz}, \mathrm{CDCl}_{3}\right) \delta 154.7,134.9,134.2,133.0,130.5,129.2,127.4,126.8,117.4$ $112.2,80.8,55.3,40.0,28.3$.

HRMS (ESI) calcd for $\mathrm{C}_{21} \mathrm{H}_{20} \mathrm{Cl}_{2} \mathrm{~N}_{2} \mathrm{NaO}_{2}[\mathrm{M}+\mathrm{H}]^{+}$425.0800, found 425.0791 . tert-Butyl 1-(4-cyano-3-methylphenyl)-3,4-dihydroisoquinoline-2(1 H)-carboxylate (3af). 


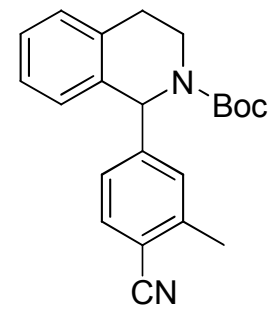

Prepared according to the general method and purified by common column chromatography $(\mathrm{PE}: \mathrm{EA}=20: 1)$ to give the title compounds as a colorless viscous oil (113 mg, yield: 81\%).

${ }^{1} \mathrm{H}$ NMR $\left(400 \mathrm{MHz}, \mathrm{CDCl}_{3}\right.$, rotamers seen) $\delta 7.50(\mathrm{~d}, J=8.0 \mathrm{~Hz}, 1 \mathrm{H}), 7.29-7.15(\mathrm{~m}, 4 \mathrm{H}), 7.12$ $(\mathrm{d}, J=8.0 \mathrm{~Hz}, 1 \mathrm{H}), 7.08-6.95(\mathrm{~m}, 1 \mathrm{H}), 6.56-6.03(\mathrm{~m}, 1 \mathrm{H}), 4.22-3.80(\mathrm{~m}, 1 \mathrm{H}), 3.32-3.15(\mathrm{~m}$, 1H), $3.03-2.87(\mathrm{~m}, 1 \mathrm{H}), 2.85-2.68(\mathrm{~m}, 1 \mathrm{H}), 2.49(\mathrm{~s}, 3 \mathrm{H}), 1.48(\mathrm{~s}, 9 \mathrm{H})$.

${ }^{13} \mathrm{C}$ NMR (100 MHz, $\mathrm{CDCl}_{3}$, rotamers seen) $\delta 154.7,148.3,142.980,135.3,134.5,132.5,129.9$, $129.1,128.3,127.4,126.4,126.0,118.1,111.6,80.5,56.9,38.9,28.5,20.6$.

HRMS (ESI) calcd for $\mathrm{C}_{22} \mathrm{H}_{24} \mathrm{~N}_{2} \mathrm{NaO}_{2}[\mathrm{M}+\mathrm{Na}]^{+}$317.1735, found 317.1727.

tert-Butyl 1-(pyridin-4-yl)-3,4-dihydroisoquinoline-2(1H)-carboxylate (3ag)<smiles>CC(=O)OC(=O)N1CCc2ccccc2C1c1ccncc1</smiles>

Prepared according to the general method and purified by common column chromatography $(\mathrm{PE}: \mathrm{EA}=20: 1)$ to give the title compounds as a colorless viscous oil (118 mg, yield: 96\%).

${ }^{1} \mathrm{H}$ NMR (400 MHz, $\mathrm{CDCl}_{3}$, rotamers seen) $\delta 8.52(\mathrm{~d}, J=3.2 \mathrm{~Hz}, 2 \mathrm{H}), 7.31-7.18(\mathrm{~m}, 3 \mathrm{H}), 7.15$ $(\mathrm{d}, J=4.4 \mathrm{~Hz}, 2 \mathrm{H}), 7.12-7.03(\mathrm{~m}, 1 \mathrm{H}), 6.49-6.00(\mathrm{~m}, 1 \mathrm{H}), 4.21-3.75(\mathrm{~m}, 1 \mathrm{H}), 3.35-3.19(\mathrm{~m}$, 1H), $3.03-2.86(\mathrm{~m}, 1 \mathrm{H}), 2.84-2.65(\mathrm{~m}, 1 \mathrm{H}), 1.49(\mathrm{~s}, 9 \mathrm{H})$.

${ }^{13} \mathrm{C}$ NMR (100 MHz, $\mathrm{CDCl}_{3}$, rotamers seen) $\delta 151.6,149.9,135.3,134.2,129.0,128.3,127.5$, $126.4,122.8,80.5,57.6,56.2,39.4,38.4,28.5,28.4$. 
HRMS (ESI) calcd for $\mathrm{C}_{19} \mathrm{H}_{23} \mathrm{~N}_{2} \mathrm{O}_{2}[\mathrm{M}+\mathrm{H}]^{+} 311.1760$, found 311.1757 .

tert-Butyl 1-(2-chloropyridin-4-yl)-3,4-dihydroisoquinoline-2(1H)-carboxylate (3ah)<smiles>CC(C)(C)OC(=O)N1CCc2ccccc2C1c1ccnc(Cl)c1</smiles>

Prepared according to the general method and purified by common column chromatography (PE:EA $=10: 1)$ to give the title compounds as a colorless viscous oil $(111 \mathrm{mg}$, yield: $80 \%)$.

${ }^{1} \mathrm{H}$ NMR (400 MHz, $\mathrm{CDCl}_{3}$, rotamers seen) $\delta 8.30(\mathrm{~d}, J=4.8 \mathrm{~Hz}, 1 \mathrm{H}), 7.33-7.00(\mathrm{~m}, 6 \mathrm{H}), 6.53-$ $5.93(\mathrm{~m}, 1 \mathrm{H}), 4.15-3.80(\mathrm{~m}, 1 \mathrm{H}), 3.37-3.22(\mathrm{~m}, 1 \mathrm{H}), 3.06-2.87(\mathrm{~m}, 1 \mathrm{H}), 2.85-2.67(\mathrm{~m}, 1 \mathrm{H})$ $1.51(\mathrm{~s}, 9 \mathrm{H})$.

${ }^{13} \mathrm{C} \mathrm{NMR}\left(100 \mathrm{MHz}, \mathrm{CDCl}_{3}\right.$, rotamers seen) $\delta 155.2,151.8,149.7,135.3,133.5,129.1,128.2$ $127.8,126.6,123.3,121.7,80.8,57.4,56.1,39.6,38.5,28.5,28.4$.

HRMS (ESI) calcd for $\mathrm{C}_{19} \mathrm{H}_{22} \mathrm{ClN}_{2} \mathrm{O}_{2}[\mathrm{M}+\mathrm{H}]^{+}$345.1370, found 345.1370.

tert-Butyl 1-(3-chloropyridin-4-yl)-3,4-dihydroisoquinoline-2(1H)-carboxylate (3ai)<smiles>CC(=O)N1CCc2ccccc2C1c1ccncc1Cl</smiles>

Prepared according to the general method and purified by common column chromatography $(\mathrm{PE}: \mathrm{EA}=5: 1)$ to give the title compounds as a colorless viscous oil (90 mg, yield: $66 \%$ ).

${ }^{1} \mathrm{H}$ NMR (400 MHz, $\mathrm{CDCl}_{3}$, rotamers seen) $\delta 8.61(\mathrm{~s}, 1 \mathrm{H}), 8.35(\mathrm{~d}, J=5.2 \mathrm{~Hz}, 1 \mathrm{H}), 7.25-7.18$ (m, 2H), $7.18-7.12(\mathrm{~m}, 1 \mathrm{H}), 7.12-6.98(\mathrm{~m}, 2 \mathrm{H}), 6.42(\mathrm{~s}, 1 \mathrm{H}), 4.30-3.96(\mathrm{~m}, 1 \mathrm{H}), 3.69-3.31$ (m, 1H), $3.11-2.80(\mathrm{~m}, 2 \mathrm{H}), 1.36(\mathrm{~s}, 9 \mathrm{H})$. 
${ }^{13} \mathrm{C}$ NMR $\left(100 \mathrm{MHz}, \mathrm{CDCl}_{3}\right.$, rotamers seen) $\delta 154.7,149.8,148.0,135.0,133.9,131.3,129.1$, $127.4,126.8,123.0,80.9,54.8,40.1,29.2,28.3$

HRMS (ESI) calcd for $\mathrm{C}_{19} \mathrm{H}_{22} \mathrm{ClN}_{2} \mathrm{O}_{2}[\mathrm{M}+\mathrm{H}]^{+} 345.1370$, found 345.1369 .

tert-Butyl 1-(2-methoxypyridin-4-yl)-3,4-dihydroisoquinoline-2(1H)-carboxylate (3aj)<smiles>COc1cc(C2c3ccccc3CCN2C(=O)OC(C)(C)C)ccn1</smiles>

Prepared according to the general method and purified by common column chromatography $(\mathrm{PE}: \mathrm{EA}=10: 1)$ to give the title compounds as a colorless viscous oil (102 mg, yield: 76\%).

${ }^{1} \mathrm{H}$ NMR $\left(400 \mathrm{MHz}, \mathrm{CDCl}_{3}\right.$, rotamers seen) $\delta 8.07(\mathrm{~d}, J=5.2 \mathrm{~Hz}, 1 \mathrm{H}), 7.33-6.96(\mathrm{~m}, 4 \mathrm{H}), 6.92-$ $6.68(\mathrm{~m}, 1 \mathrm{H}), 6.62-6.42(\mathrm{~m}, 1 \mathrm{H}), 6.41-5.95(\mathrm{~m}, 1 \mathrm{H}), 4.18-3.95(\mathrm{~m}, 1 \mathrm{H}), 3.89(\mathrm{~s}, 3 \mathrm{H}), 3.39-$ $3.20(\mathrm{~m}, 1 \mathrm{H}), 3.03-2.83(\mathrm{~m}, 1 \mathrm{H}), 2.82-2.63(\mathrm{~m}, 1 \mathrm{H}), 1.49(\mathrm{~s}, 9 \mathrm{H})$.

${ }^{13} \mathrm{C} \mathrm{NMR}\left(100 \mathrm{MHz}, \mathrm{CDCl}_{3}\right.$, rotamers seen) $\delta 164.4,154.5,146.8,135.3,134.4,134.2,129.0$ $128.3,127.5,126.3,116.4,110.0,80.6,80.3,57.4,56.2,53.4,39.6,38.2,28.5,28.3$.

HRMS (ESI) calcd for $\mathrm{C}_{20} \mathrm{H}_{25} \mathrm{~N}_{2} \mathrm{O}_{3}[\mathrm{M}+\mathrm{H}]^{+} 341.1865$, found 341.1862.

tert-Butyl 1-(4-cyanophenyl)-6,7-dimethoxy-3,4-dihydroisoquinoline-2(1H)-carboxylate (3bb)<smiles>COc1cc2c(cc1OC)C(c1ccc(C#N)cc1)N(C(=O)OC(C)(C)C)CC2</smiles>

Prepared according to the general method and purified by common column chromatography $(\mathrm{PE}: \mathrm{EA}=5: 1)$ to give the title compounds as a white solid $(128 \mathrm{mg}$, yield: $81 \%)$, m.p. $=$ $127-128^{\circ} \mathrm{C}$. 
${ }^{1} \mathrm{H}$ NMR (400 MHz, $\mathrm{CDCl}_{3}$, rotamers seen) $\delta 7.58(\mathrm{~d}, J=8.0 \mathrm{~Hz}, 2 \mathrm{H}), 7.36(\mathrm{~d}, J=8.0 \mathrm{~Hz}, 2 \mathrm{H})$, $6.68(\mathrm{~s}, 1 \mathrm{H}), 6.54-6.03(\mathrm{~m}, 2 \mathrm{H}), 4.20-3.95(\mathrm{~m}, 1 \mathrm{H}), 3.89(\mathrm{~s}, 3 \mathrm{H}), 3.76(\mathrm{~s}, 3 \mathrm{H}), 3.13-3.00(\mathrm{~m}$ $1 \mathrm{H}), 3.00-2.82(\mathrm{~m}, 1 \mathrm{H}), 2.75-2.57(\mathrm{~m}, 1 \mathrm{H}), 1.50(\mathrm{~s}, 9 \mathrm{H})$.

${ }^{13} \mathrm{C}$ NMR (100 MHz, $\mathrm{CDCl}_{3}$, rotamers seen) $\delta 154.9,148.5,147.8,132.2,129.1,127.6,125.9$, $118.9,111.6,111.3,111.0,80.6,57.5,56.5,56.2,56.1,38.8,37.6,28.6,28.1$.

HRMS (ESI) calcd for $\mathrm{C}_{23} \mathrm{H}_{26} \mathrm{~N}_{2} \mathrm{NaO}_{4}[\mathrm{M}+\mathrm{Na}]^{+}$417.1790, found 417.1792.

tert-Butyl 1-(4-cyanophenyl)-6-methoxy-3,4-dihydroisoquinoline-2(1H)-carboxylate (3cb)<smiles>COc1ccc2c(c1)CCN(C(=O)OCc1ccccc1)C2c1ccc(C#N)cc1</smiles>

Prepared according to the general method and purified by common column chromatography (PE:EA = 20:1) to give the title compounds as a colorless viscous oil (109 mg, yield: 75\%).

${ }^{1} \mathrm{H}$ NMR $\left(400 \mathrm{MHz}, \mathrm{CDCl}_{3}\right.$, rotamers seen) $\delta 7.57(\mathrm{~d}, J=8.0 \mathrm{~Hz}, 2 \mathrm{H}), 7.34(\mathrm{~d}, J=8.0 \mathrm{~Hz}, 2 \mathrm{H})$, $6.93(\mathrm{~s}, 1 \mathrm{H}), 6.81-6.69(\mathrm{~m}, 2 \mathrm{H}), 6.47-6.00(\mathrm{~m}, 1 \mathrm{H}), 4.15-3.86(\mathrm{~m}, 1 \mathrm{H}), 3.81(\mathrm{~s}, 3 \mathrm{H}), 3.27-$ $3.09(\mathrm{~m}, 1 \mathrm{H}), 3.03-2.82(\mathrm{~m}, 1 \mathrm{H}), 2.81-2.60(\mathrm{~m}, 1 \mathrm{H}), 1.48(\mathrm{~s}, 9 \mathrm{H})$

${ }^{13} \mathrm{C}$ NMR (100 MHz, $\mathrm{CDCl}_{3}$, rotamers seen) $\delta 158.7,148.8,136.6,132.1,129.3,128.7,126.5$, $118.8,113.6,112.7,111.0,80.5,57.1,56.3,55.3,38.8,38.1,28.8,28.5$.

HRMS (ESI) calcd for $\mathrm{C}_{22} \mathrm{H}_{24} \mathrm{~N}_{2} \mathrm{NaO}_{3}[\mathrm{M}+\mathrm{Na}]^{+}$387.1685, found 387.1675.

tert-Butyl 5-bromo-1-(4-cyanophenyl)-3,4-dihydroisoquinoline-2(1H)-carboxylate (3db) 
<smiles>CC(C)(C)OC(=O)N1CCc2c(Br)cccc2C1c1ccc(C#N)cc1</smiles>

Prepared according to the general method and purified by common column chromatography (PE:EA $=20: 1)$ to give the title compounds as a colorless viscous oil (69 mg, yield: $42 \%$ ).

${ }^{1} \mathrm{H}$ NMR (400 MHz, $\mathrm{CDCl}_{3}$, rotamers seen) $\delta 7.66-7.56(\mathrm{~m}, 2 \mathrm{H}), 7.56-7.42(\mathrm{~m}, 1 \mathrm{H}), 7.42-$ $7.29(\mathrm{~m}, 2 \mathrm{H}), 7.09(\mathrm{t}, J=7.6 \mathrm{~Hz}, 1 \mathrm{H}), 7.03-6.94(\mathrm{~m}, 1 \mathrm{H}), 6.45(\mathrm{~s}, 1 \mathrm{H}), 4.34-3.97(\mathrm{~m}, 1 \mathrm{H}), 3.12$ $-3.00(\mathrm{~m}, 1 \mathrm{H}), 2.99-2.74(\mathrm{~m}, 2 \mathrm{H}), 1.60$ and $1.50(\mathrm{~s}, 9 \mathrm{H})$.

${ }^{13} \mathrm{C}$ NMR $\left(100 \mathrm{MHz}, \mathrm{CDCl}_{3}\right) \delta 147.7,136.5,135.8,135.1,135.0,132.2,131.5,129.9,129.1$, $128.3,127.8,127.7,127.5,125.6,118.6,111.4,83.5,80.8,56.7,43.5,38.0,29.2,28.5$.

HRMS (ESI) calcd for $\mathrm{C}_{21} \mathrm{H}_{21} \mathrm{BrN}_{2} \mathrm{NaO}_{2}[\mathrm{M}+\mathrm{Na}]^{+}$435.0684, found 435.0673.

tert-Butyl 6-bromo-1-(4-cyanophenyl)-3,4-dihydroisoquinoline-2(1H)-carboxylate (3eb)<smiles>CC(C)(C)OC(=O)N1CCc2cc(Br)ccc2C1c1ccc(C#N)cc1</smiles>

Prepared according to the general method and purified by common column chromatography (PE:EA $=20: 1)$ to give the title compounds as a colorless viscous oil (105 mg, yield: 63\%).

${ }^{1} \mathrm{H}$ NMR (400 MHz, $\mathrm{CDCl}_{3}$, rotamers seen) $\delta 7.58(\mathrm{~d}, J=8.0 \mathrm{~Hz}, 2 \mathrm{H}), 7.41-7.36(\mathrm{~m}, 1 \mathrm{H}), 7.36-$ $7.29(\mathrm{~m}, 3 \mathrm{H}), 6.90(\mathrm{~d}, J=7.6 \mathrm{~Hz}, 1 \mathrm{H}), 6.51-6.01(\mathrm{~m}, 1 \mathrm{H}), 4.21-3.81(\mathrm{~m}, 1 \mathrm{H}), 3.23-3.09(\mathrm{~m}$, 1H), $3.02-2.86(\mathrm{~m}, 1 \mathrm{H}), 2.82-2.65(\mathrm{~m}, 1 \mathrm{H}), 1.48(\mathrm{~s}, 9 \mathrm{H})$.

${ }^{13} \mathrm{C}$ NMR (100 MHz, $\mathrm{CDCl}_{3}$, rotamers seen) $\delta 154.4,147.8,137.6,133.4,132.8,132.2,131.9$, 
$129.9,129.6,128.8,121.2,118.6,111.4,80.8,56.2,38.2,28.5,28.3$.

HRMS (ESI) calcd for $\mathrm{C}_{21} \mathrm{H}_{21} \mathrm{BrN}_{2} \mathrm{NaO}_{2}[\mathrm{M}+\mathrm{Na}]^{+}$435.0684, found 435.0678.

tert-Butyl 7-bromo-1-(4-cyanophenyl)-3,4-dihydroisoquinoline-2(1H)-carboxylate (3fb)<smiles>CC(C)(C)OC(=O)N1CCc2ccc(Br)cc2C1c1ccc(C#N)cc1</smiles>

Prepared according to the general method and purified by common column chromatography (PE:EA $=20: 1)$ to give the title compounds as a colorless viscous oil (79 mg, yield: $48 \%$ ).

${ }^{1} \mathrm{H}$ NMR (400 MHz, $\mathrm{CDCl}_{3}$, rotamers seen) $\delta 7.60(\mathrm{~d}, J=8.0 \mathrm{~Hz}, 2 \mathrm{H}), 7.43-7.29(\mathrm{~m}, 3 \mathrm{H}), 7.17$ (s, 1H), $7.10(\mathrm{~d}, J=8.0 \mathrm{~Hz}, 1 \mathrm{H}), 6.53-6.04(\mathrm{~m}, 1 \mathrm{H}), 4.24-3.78(\mathrm{~m}, 1 \mathrm{H}), 3.25-3.08(\mathrm{~m}, 1 \mathrm{H})$, $2.99-2.82(\mathrm{~m}, 1 \mathrm{H}), 2.79-2.64(\mathrm{~m}, 1 \mathrm{H}), 1.48(\mathrm{~s}, 9 \mathrm{H})$.

${ }^{13} \mathrm{C}$ NMR $\left(100 \mathrm{MHz}, \mathrm{CDCl}_{3}\right) \delta 154.7,147.6,136.5,135.0,134.3,132.3,131.0,130.8,130.6$, $128.8,127.9,120.0,118.6,111.5,80.8,56.9,38.0,28.5,28.0$.

HRMS (ESI) calcd for $\mathrm{C}_{21} \mathrm{H}_{21} \mathrm{BrN}_{2} \mathrm{NaO}_{2}[\mathrm{M}+\mathrm{Na}]^{+}$435.0684, found 435.0671 .

tert-Butyl

1-(4-cyanophenyl)-9-methyl-3,4-dihydro-1 $H$-pyrido[3,4-b]indole-2(9H)-carboxylate (3gb)

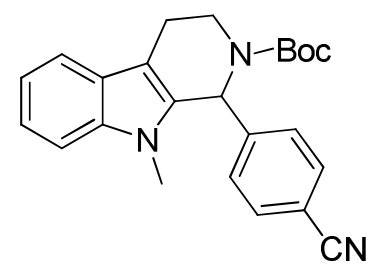

Prepared according to the general method and purified by common column chromatography (PE:EA $=20: 1)$ to give the title compounds as a white solid $(141 \mathrm{mg}$, yield: $91 \%)$, m.p. $=$ $91-92^{\circ} \mathrm{C}$. 
${ }^{1} \mathrm{H}$ NMR (400 MHz, $\mathrm{CDCl}_{3}$, rotamers seen) $\delta 7.67-7.53(\mathrm{~m}, 3 \mathrm{H}), 7.40(\mathrm{~d}, J=7.6 \mathrm{~Hz}, 2 \mathrm{H}), 7.33-$ $7.22(\mathrm{~m}, 3 \mathrm{H}), 7.16(\mathrm{t}, J=7.2 \mathrm{~Hz}, 1 \mathrm{H}), 6.68-6.31(\mathrm{~m}, 1 \mathrm{H}), 4.31-4.07(\mathrm{~m}, 1 \mathrm{H}), 3.36(\mathrm{~s}, 3 \mathrm{H}), 3.04$ $-2.85(\mathrm{~m}, 2 \mathrm{H}), 2.81(\mathrm{t}, J=11.2 \mathrm{~Hz}, 1 \mathrm{H}), 1.59$ and $1.50(\mathrm{~s}, 9 \mathrm{H})$.

${ }^{13} \mathrm{C}$ NMR (100 MHz, $\mathrm{CDCl}_{3}$, rotamers seen) $\delta 155.0,145.4,137.4,132.5,131.8,129.2,126.4$, $122.1,119.5,118.5,112.0,109.9,109.1,80.7,53.5,52.3,38.2,31.6,29.9,28.5,22.7,21.4,14.2$.

HRMS (ESI) calcd for $\mathrm{C}_{24} \mathrm{H}_{26} \mathrm{~N}_{3} \mathrm{O}_{2}[\mathrm{M}+\mathrm{H}]^{+}$388.2025, found 388.2021.

\section{Benzyl 1-(4-cyanophenyl)-3,4-dihydroisoquinoline-2(1H)-carboxylate (3hb)}<smiles>N#Cc1ccc(C2c3ccccc3CCN2C(=O)OCc2ccccc2)cc1</smiles>

Prepared according to the general method and purified by common column chromatography $(\mathrm{PE}: \mathrm{EA}=20: 1)$ to give the title compounds as a colorless viscous oil (101 mg, yield: 69\%).

${ }^{1} \mathrm{H}$ NMR (400 MHz, $\mathrm{CDCl}_{3}$, rotamers seen) $\delta 7.64-7.47(\mathrm{~m}, 2 \mathrm{H}), 7.42-7.29(\mathrm{~m}, 6 \mathrm{H}), 7.29-$ $7.15(\mathrm{~m}, 4 \mathrm{H}), 7.11-6.91(\mathrm{~m}, 1 \mathrm{H}), 6.56-6.17(\mathrm{~m}, 1 \mathrm{H}), 5.36-5.04(\mathrm{~m}, 2 \mathrm{H}), 4.25-3.93(\mathrm{~m}, 1 \mathrm{H})$ $3.38-3.19(\mathrm{~m}, 1 \mathrm{H}), 3.10-2.89(\mathrm{~m}, 1 \mathrm{H}), 2.85-2.66(\mathrm{~m}, 1 \mathrm{H})$

${ }^{13} \mathrm{C}$ NMR $\left(100 \mathrm{MHz}, \mathrm{CDCl}_{3}\right.$, rotamers seen) $\delta 155.6,147.9,136.4,135.0,134.0,132.2,129.0$ $128.7,128.3,127.9,127.7,126.6,118.8,111.3,67.7,57.5,38.9,28.4$.

HRMS (ESI) calcd for $\mathrm{C}_{24} \mathrm{H}_{21} \mathrm{~N}_{2} \mathrm{O}_{2}[\mathrm{M}+\mathrm{H}]^{+}$369.1603, found 369.1601.

Ethyl 1-(4-cyanophenyl)-3,4-dihydroisoquinoline-2(1H)-carboxylate (3ib) 
<smiles>CCOC(=O)N1CCc2ccccc2C1c1ccc(C#N)cc1</smiles>

Prepared according to the general method and purified by common column chromatography (PE:EA $=10: 1)$ to give the title compounds as a colorless viscous oil ( $80 \mathrm{mg}$, yield: $65 \%$ ).

${ }^{1} \mathrm{H}$ NMR (400 MHz, $\mathrm{CDCl}_{3}$, rotamers seen) $\delta 7.57(\mathrm{~d}, J=8.0 \mathrm{~Hz}, 2 \mathrm{H}), 7.35(\mathrm{~d}, J=8.0 \mathrm{~Hz}, 2 \mathrm{H})$, $7.30-7.15(\mathrm{~m}, 3 \mathrm{H}), 7.02(\mathrm{~d}, J=6.4 \mathrm{~Hz}, 1 \mathrm{H}), 6.53-6.17(\mathrm{~m}, 1 \mathrm{H}), 4.32-3.87(\mathrm{~m}, 3 \mathrm{H}), 3.24(\mathrm{t}, J$ $=9.6 \mathrm{~Hz}, 1 \mathrm{H}), 3.07-2.90(\mathrm{~m}, 1 \mathrm{H}), 2.83-2.69(\mathrm{~m}, 1 \mathrm{H}), 1.29(\mathrm{t}, J=7.2 \mathrm{~Hz}, 3 \mathrm{H})$.

${ }^{13} \mathrm{C}$ NMR (100 MHz, $\mathrm{CDCl}_{3}$, rotamers seen) $\delta 155.9,148.0,144.3,135.1,134.1,132.2,131.8$, 131.1, 129.0, 128.3, 127.6, 126.5, 118.7, 111.3, 61.9, 57.3, 38.6, 28.3, 14.7.

HRMS (ESI) calcd for $\mathrm{C}_{19} \mathrm{H}_{19} \mathrm{~N}_{2} \mathrm{O}_{2}[\mathrm{M}+\mathrm{H}]^{+}$307.1447, found 307.1445.

\section{(3S)-2-tert-butyl 3-methyl 1-(4-cyanophenyl)-3,4-dihydroisoquinoline-2,3(1H)-dicarboxylate}

\section{$(3 \mathbf{j b})$}<smiles>CC(=O)OC(C)=O</smiles>

Prepared according to the general method and purified by common column chromatography $(\mathrm{PE}: \mathrm{EA}=5: 1)$ to give the title compounds as a colorless viscous oil $(109 \mathrm{mg}$, yield: $70 \%)$, d.r. $=$ 1.2:1 (inseparable).

${ }^{1} \mathrm{H}$ NMR (400 MHz, $\mathrm{CDCl}_{3}$, rotamers seen) $\delta 7.61-7.51(\mathrm{~m}, 2 \mathrm{H}), 7.46-7.27(\mathrm{~m}, 3 \mathrm{H}), 7.25-$ $7.14(\mathrm{~m}, 2 \mathrm{H}), 7.13-7.05(\mathrm{~m}, 1 \mathrm{H}), 6.40-5.99(\mathrm{~m}, 1 \mathrm{H}), 5.31-4.86(\mathrm{~m}, 1 \mathrm{H}), 3.64-3.47(\mathrm{~m}, 3 \mathrm{H})$, 
$3.35-3.01(\mathrm{~m}, 2 \mathrm{H}), 1.48-1.27(\mathrm{~m}, 9 \mathrm{H})$.

${ }^{13} \mathrm{C}$ NMR $\left(100 \mathrm{MHz}, \mathrm{CDCl}_{3}\right.$, rotamers seen) $\delta 172.3,155.3,150.7,148.6,136.3,132.3,131.5$, $130.9,128.6,128.1,128.0,127.6,127.5,127.0,126.7,118.8,110.7,81.4,60.4,58.7,55.8,54.7$ $52.4,52.2,31.6,28.2,28.1$.

HRMS (ESI) calcd for $\mathrm{C}_{23} \mathrm{H}_{25} \mathrm{~N}_{2} \mathrm{O}_{4}[\mathrm{M}+\mathrm{H}]^{+}$393.1814, found 393.1801.

tert-Butyl 1-(4-cyanophenyl)isoindoline-2-carboxylate (3kb).

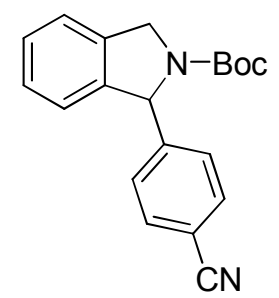

Prepared according to the general method and purified by common column chromatography $(\mathrm{PE}: \mathrm{EA}=10: 1)$ to give the title compounds as a colorless viscous oil (79 mg, yield: 62\%).

${ }^{1} \mathrm{H}$ NMR $\left(400 \mathrm{MHz}, \mathrm{CDCl}_{3}\right.$, rotamers seen) $\delta 7.66-7.56(\mathrm{~m}, 2 \mathrm{H}), 7.41-7.27(\mathrm{~m}, 4 \mathrm{H}), 7.25-$ $7.18(\mathrm{~m}, 1 \mathrm{H}), 7.03-6.89(\mathrm{~m}, 1 \mathrm{H}), 6.06-5.86(\mathrm{~m}, 1 \mathrm{H}), 4.96-4.84(\mathrm{~m}, 2 \mathrm{H}), 1.47$ and $1.24(\mathrm{~s}$, $9 \mathrm{H})$.

${ }^{13} \mathrm{C}$ NMR (100 MHz, $\mathrm{CDCl}_{3}$, rotamers seen) $\delta 154.2,154.0,149.5,148.6,140.4,140.1,136.2$ $135.7,132.6,132.4,128.2,127.9,127.3,123.4,123.1,122.8,118.8,111.2,80.4,67.1,66.9,53.01$, $52.7,28.5,28.2$.

HRMS (ESI) calcd for $\mathrm{C}_{20} \mathrm{H}_{20} \mathrm{~N}_{2} \mathrm{NaO}_{2}[\mathrm{M}+\mathrm{Na}]^{+}$343.1422, found 343.1414. 
Procedure for the synthesis 3ab under sunlight on a gram scale:

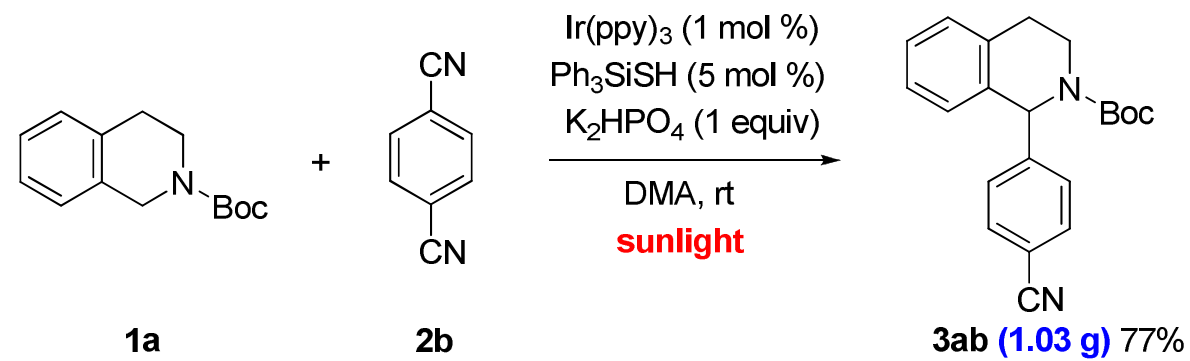

An $50 \mathrm{~mL}$ oven dried Schlenk tube was charged with substrate 1 (1.87 g, 8 mmol, 2 equiv), 2

(0.51 g, 4 mmol, 1 equiv), $\mathrm{K}_{2} \mathrm{HPO}_{4}$ (0.70 g, 4 mmol, 1 equiv), $\mathrm{Ph}_{3} \mathrm{SiSH}$ (59 mg, $0.2 \mathrm{mmol}, 5$ mol \%), Ir(ppy) $3(26 \mathrm{mg}, 40 \mu \mathrm{mol}, 1 \mathrm{~mol} \%)$ and DMA $(20 \mathrm{~mL})$. The tube was evacuated and backfilled with argon (this process was repeated three times). The reaction mixture was stirred under direct sunlight (as the picture shows in sunny days) at room temperature for 3 days (9:00 Am to 16:00 Pm every day) until a presistent green/yellow fluorescence was generally observed. The reaction mixture was diluted with water, extracted with EtOAc $(3 \times 50 \mathrm{~mL})$, combined organic layers were washed with brine, dried by anhydrous $\mathrm{Na}_{2} \mathrm{SO}_{4}$, and concentrated in vacuo. The crude product was purified by common column chromatography (PE:EA $=20: 1)$ to give 3ab $1.03 \mathrm{~g}$ as a colorless viscous oil in $77 \%$ yield.

\section{(Caution: cyanide generated during the course of the reaction)}

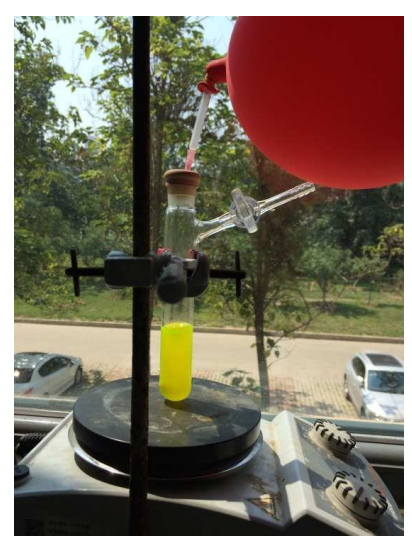

Reaction under direct sunlight. 
Synthesis and characterization of 4-(1,2,3,4-tetrahydroisoquinolin-1-yl)benzonitrile (4):

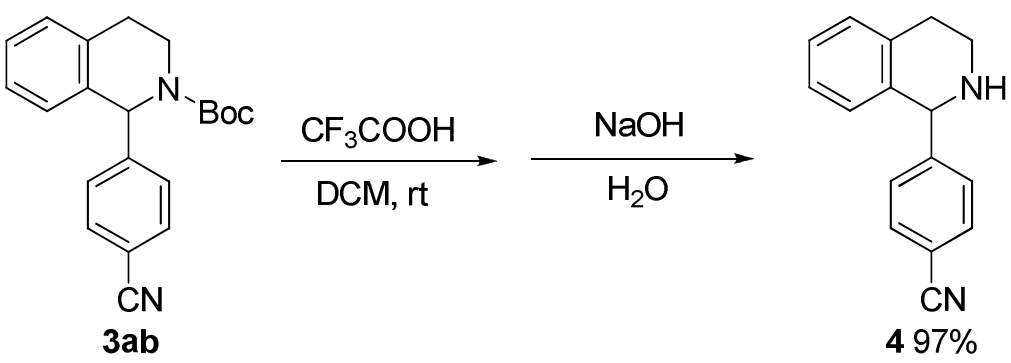

To a solution of $3 \mathbf{a b}(1.0 \mathrm{~g}, 3 \mathrm{mmol})$ in $\mathrm{DCM}(5 \mathrm{~mL})$ was added $\mathrm{CF}_{3} \mathrm{COOH}(2.2 \mathrm{~mL})$. The reaction mixture was stirred at room temperature for $4 \mathrm{~h}$. The solvent then removed under reduced pressure, and $\mathrm{NaOH} 1 \mathrm{~N}$ was added to the crude mixture. The reaction mixture was extracted with DCM. The combined organic layers were washed with brine, dried by anhydrous $\mathrm{Na}_{2} \mathrm{SO}_{4}$, and concentrated in vacuo. The crude product was purified by common column chromatography (PE:EA $=1: 1$ ) to afford product $40.68 \mathrm{~g}$ as a colorless oil in $97 \%$ yield.

${ }^{1} \mathrm{H}$ NMR $\left(400 \mathrm{MHz}, \mathrm{CDCl}_{3}\right) \delta 7.61(\mathrm{~d}, J=8.0 \mathrm{~Hz}, 2 \mathrm{H}), 7.40(\mathrm{~d}, J=8.0 \mathrm{~Hz}, 2 \mathrm{H}), 7.21-7.12(\mathrm{~m}$, 2H), $7.12-7.00(\mathrm{~m}, 1 \mathrm{H}), 6.67(\mathrm{~d}, J=7.6 \mathrm{~Hz}, 1 \mathrm{H}), 5.15(\mathrm{~s}, 1 \mathrm{H}), 3.31-3.17(\mathrm{~m}, 1 \mathrm{H}), 3.15-2.98$ (m, 2H), $2.93-2.76(\mathrm{~m}, 1 \mathrm{H}), 1.98(\mathrm{~s}, 1 \mathrm{H})$.

${ }^{13} \mathrm{C}$ NMR $\left(100 \mathrm{MHz}, \mathrm{CDCl}_{3}\right) \delta 150.3,136.8,135.5,132.3,129.8,129.4,127.9,126.8,125.9$, $118.9,111.2,61.6,42.1,29.6$.

HRMS (ESI) calcd for $\mathrm{C}_{16} \mathrm{H}_{15} \mathrm{~N}_{2}[\mathrm{M}+\mathrm{H}]^{+} 235.1235$, found 235.1234.

\section{References}

[S1] Dang, H.-S.; Roberts, B. P.; Tocher, D. A. J. Chem. Soc., Perkin Trans. 1, 2001, 2452. 


\section{Copies of ${ }^{1} \mathrm{H}$ NMR and ${ }^{13} \mathrm{C}$ NMR spectra for 3 and 4}

${ }^{1} \mathrm{H}$ NMR spectrum of compound $\mathbf{3 a a}$.
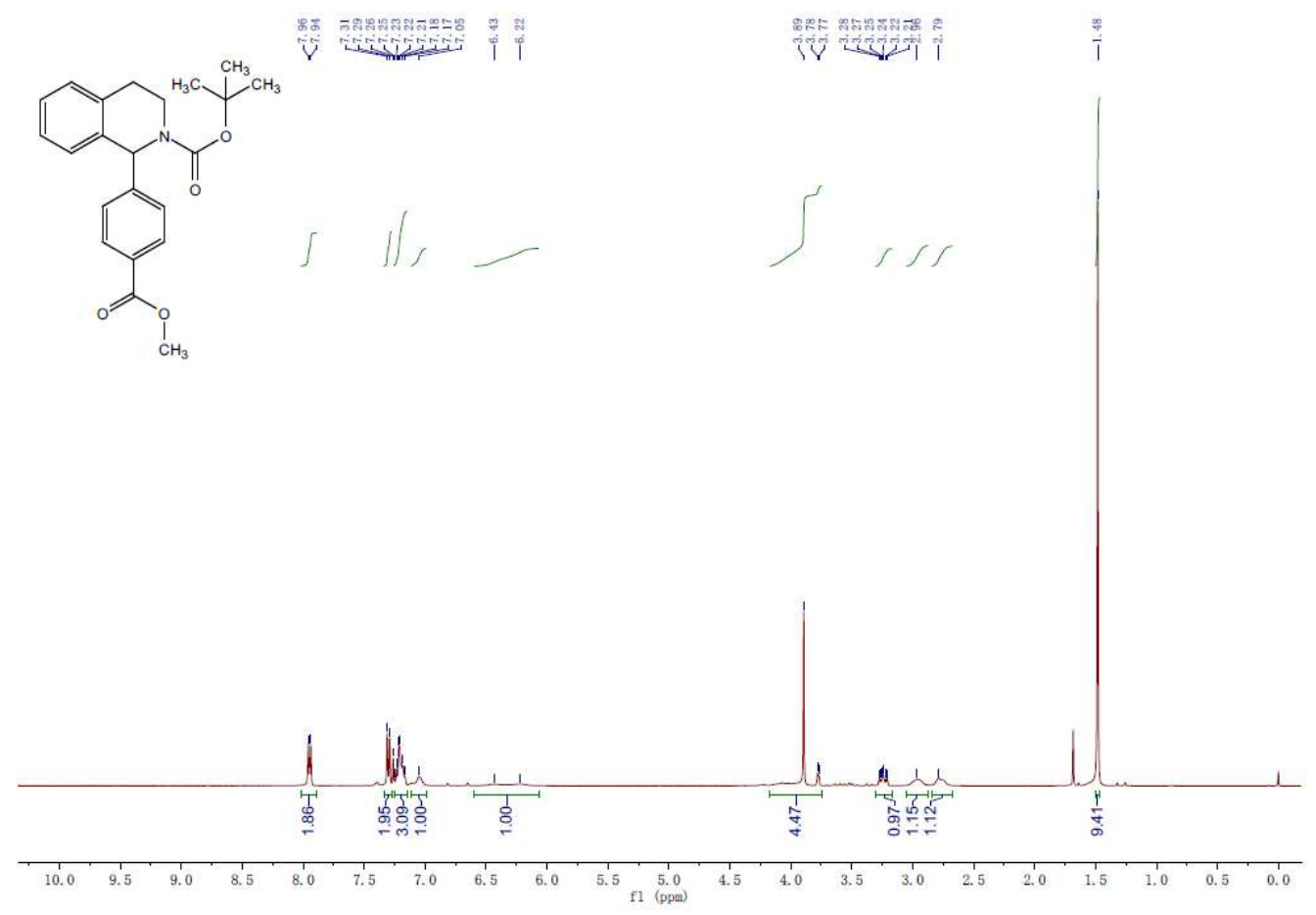

${ }^{13} \mathrm{C}$ NMR spectrum of compound 3aa.

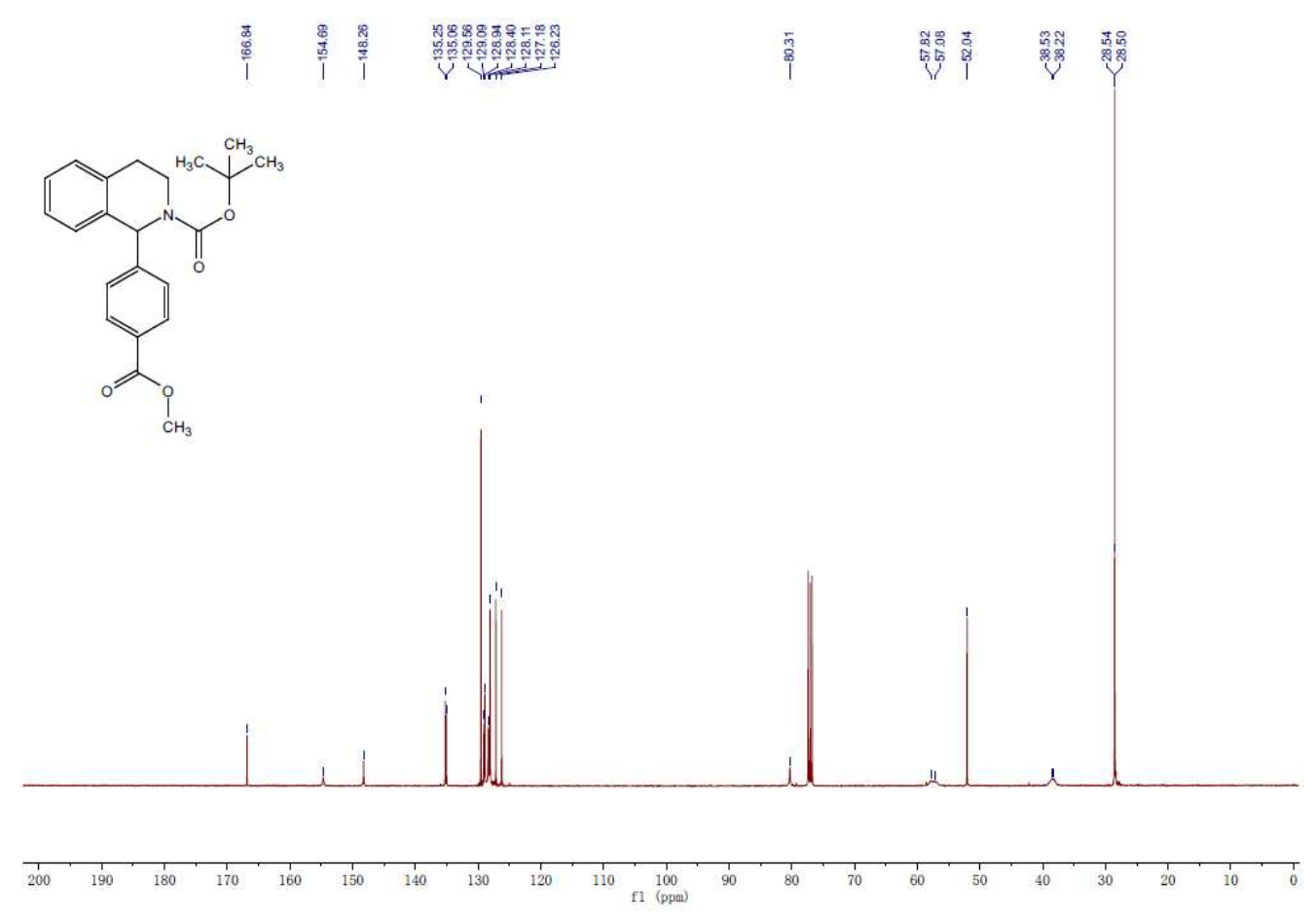


${ }^{1} \mathrm{H}$ NMR spectrum of compound $\mathbf{3 a b}$.
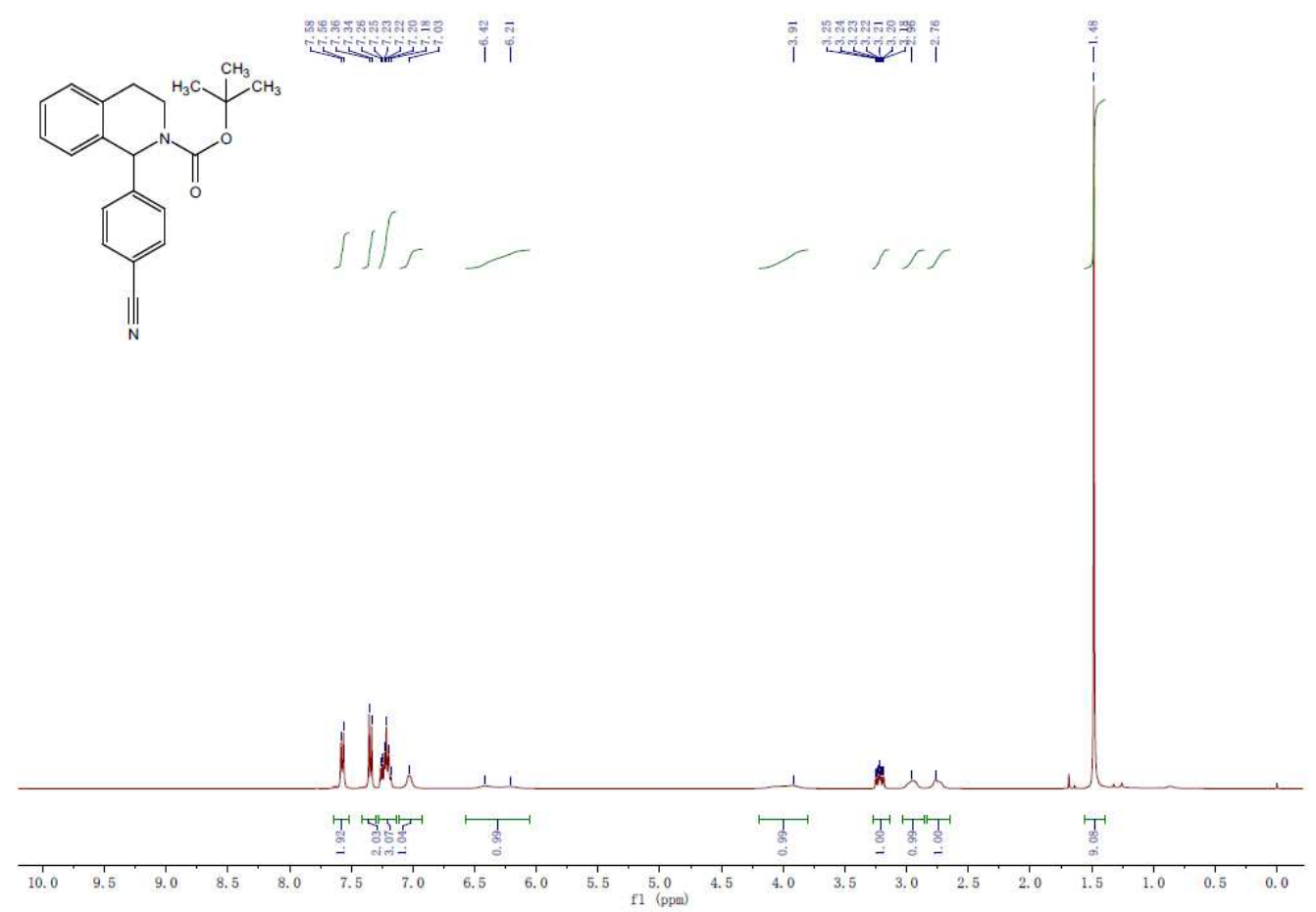

${ }^{13} \mathrm{C}$ NMR spectrum of compound $\mathbf{3 a b}$.

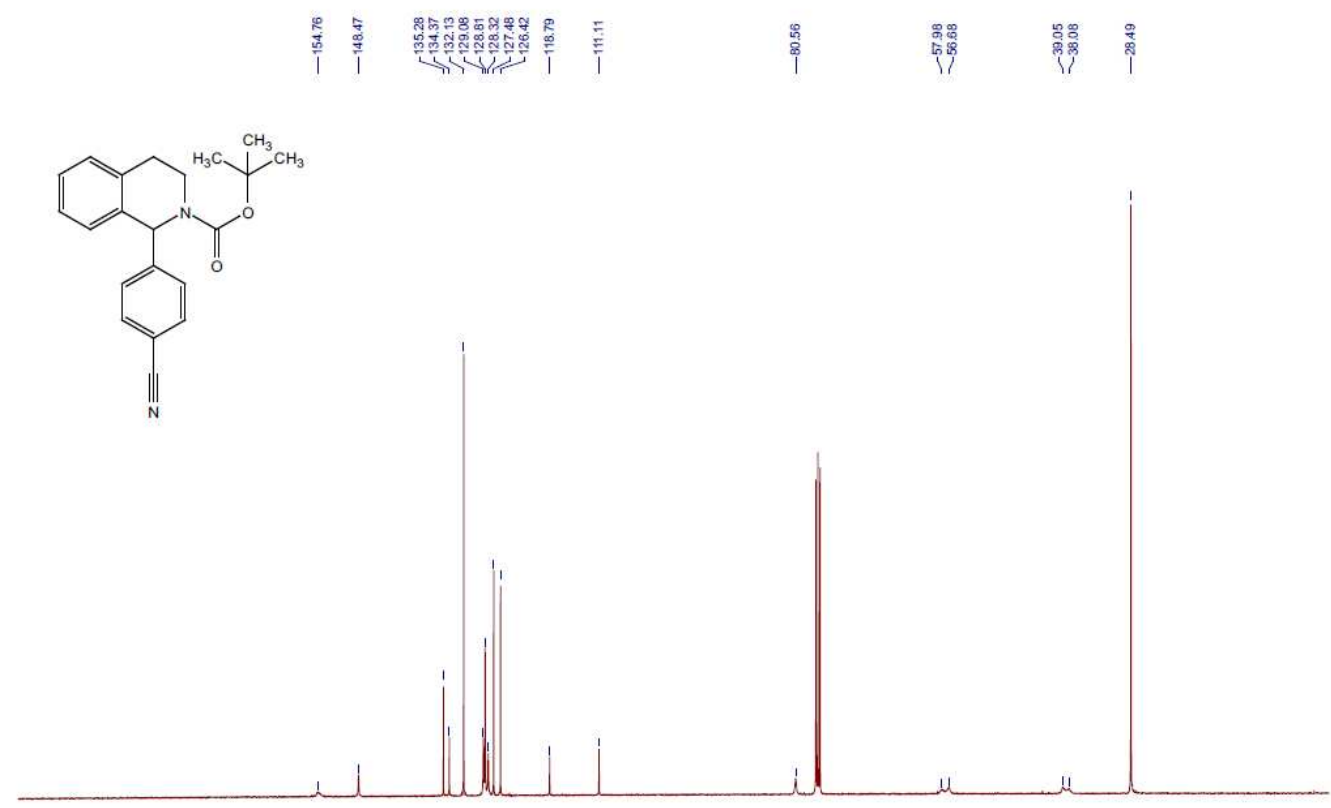

$\frac{T}{200}$

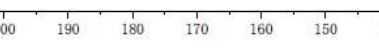

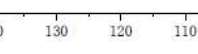

$\underset{f 1}{100}(\mathrm{ppw})$ 
${ }^{1} \mathrm{H}$ NMR spectrum of compound 3ac.

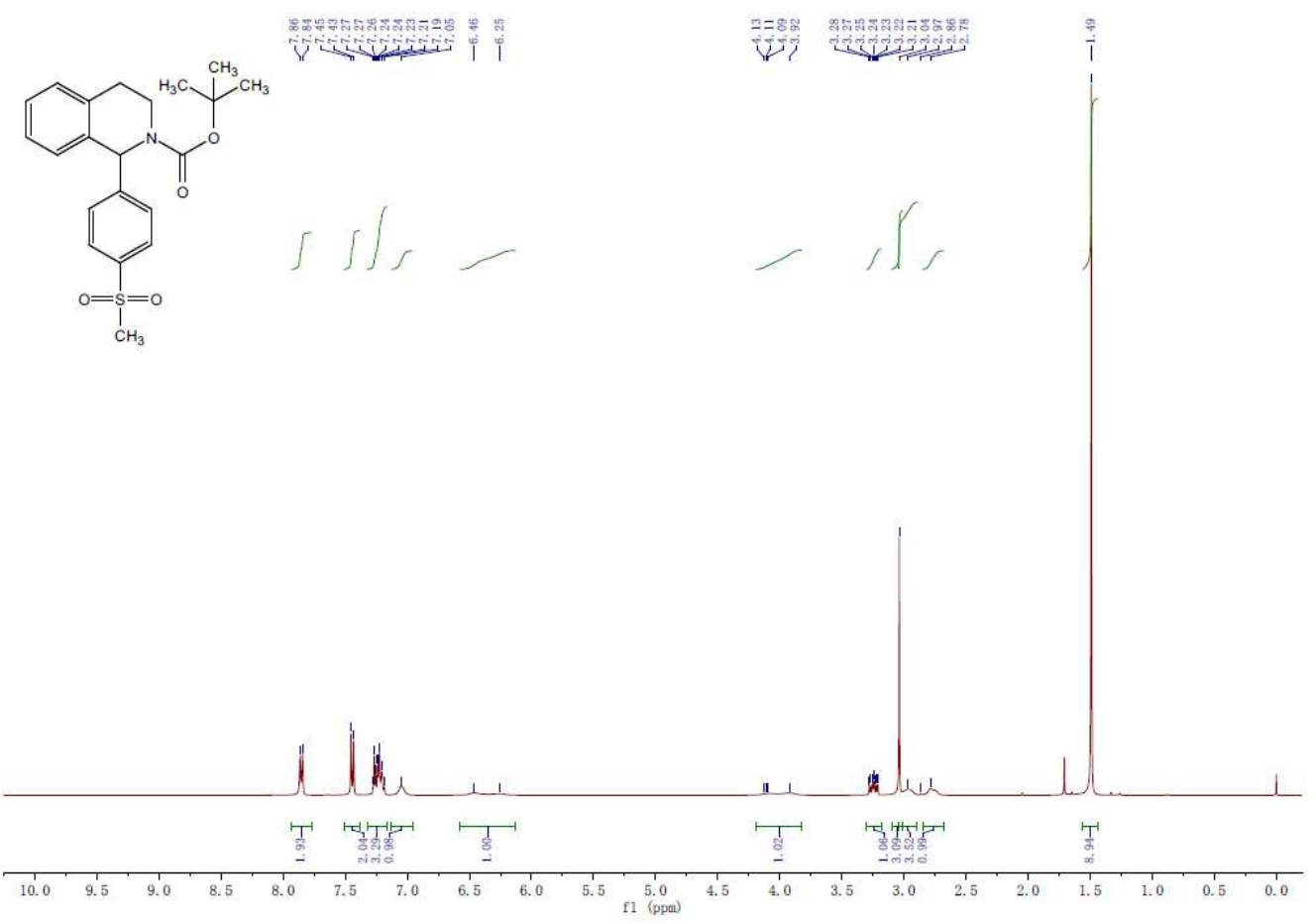

${ }^{13} \mathrm{C}$ NMR spectrum of compound 3ac.
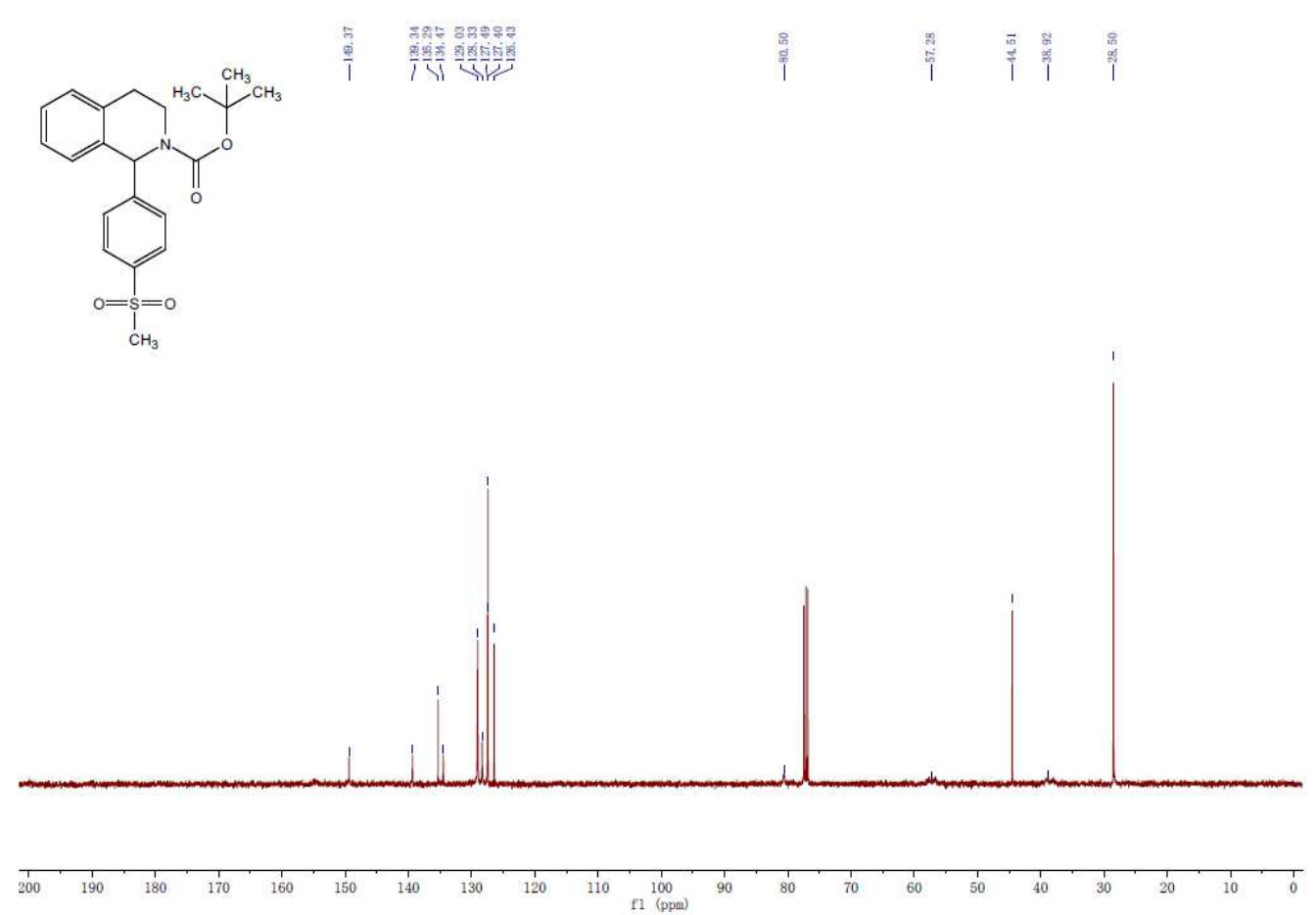
${ }^{1} \mathrm{H}$ NMR spectrum of compound 3ad.

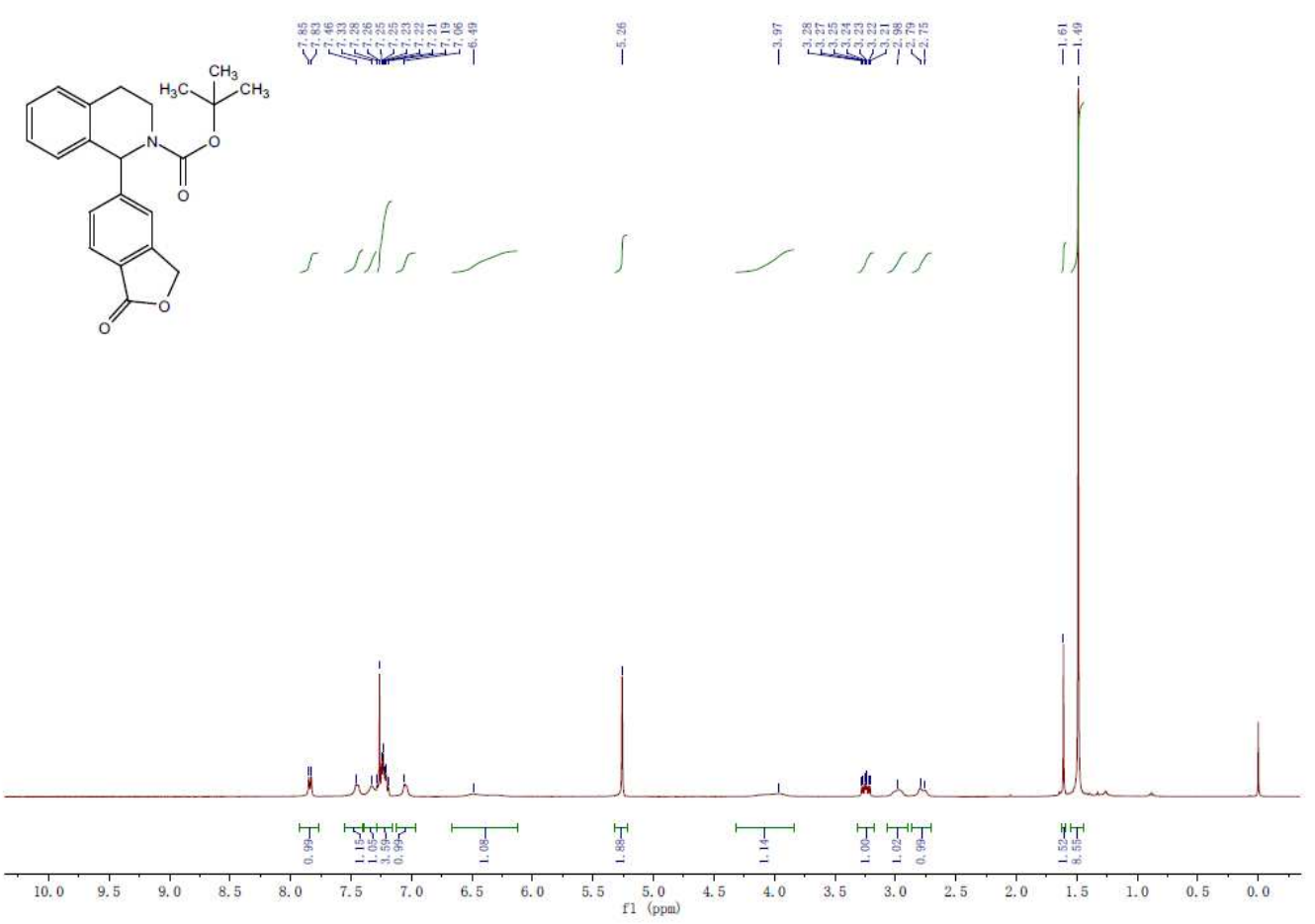

${ }^{13} \mathrm{C}$ NMR spectrum of compound $\mathbf{3 a d}$.
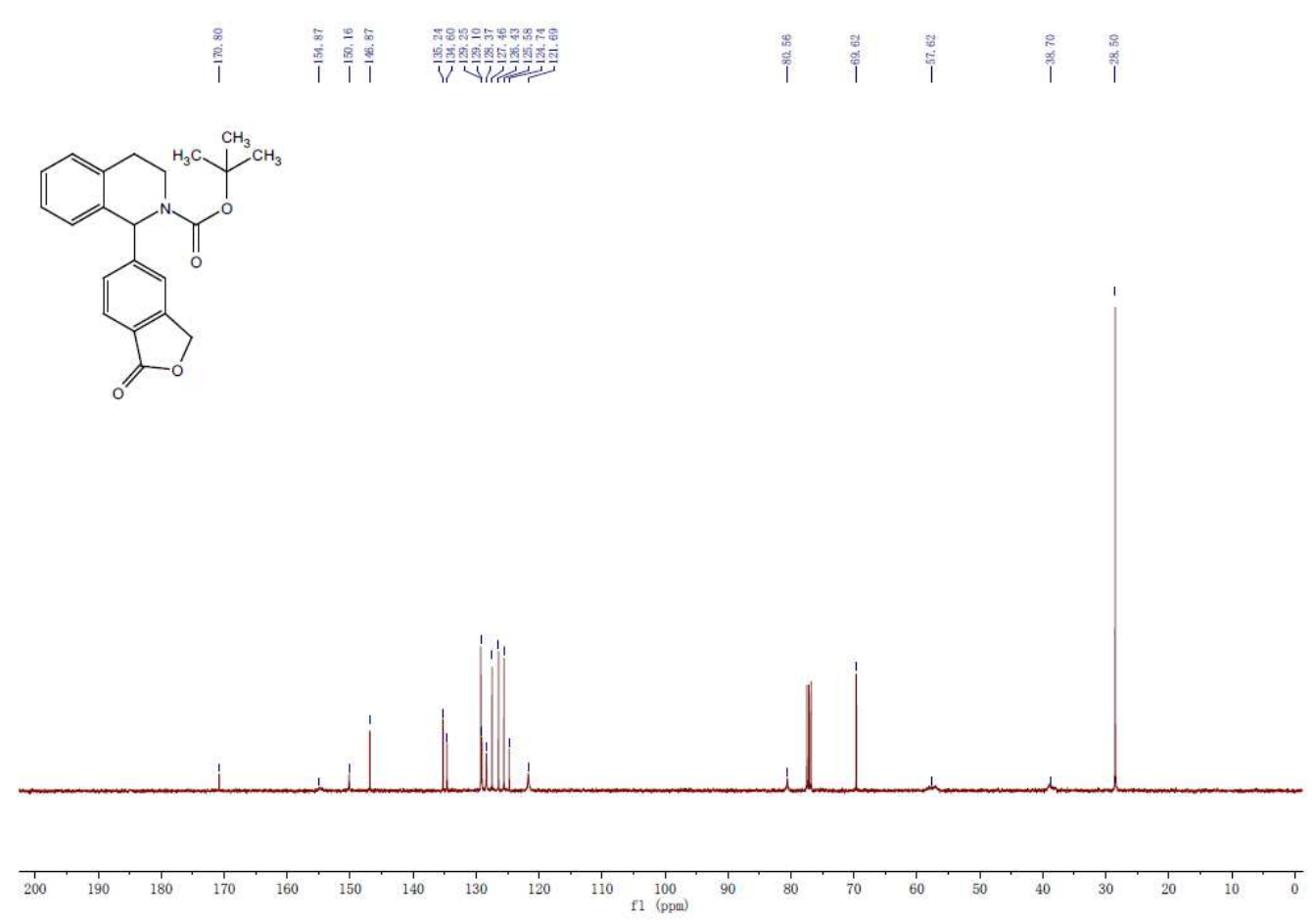
${ }^{1} \mathrm{H}$ NMR spectrum of compound 3 ae.

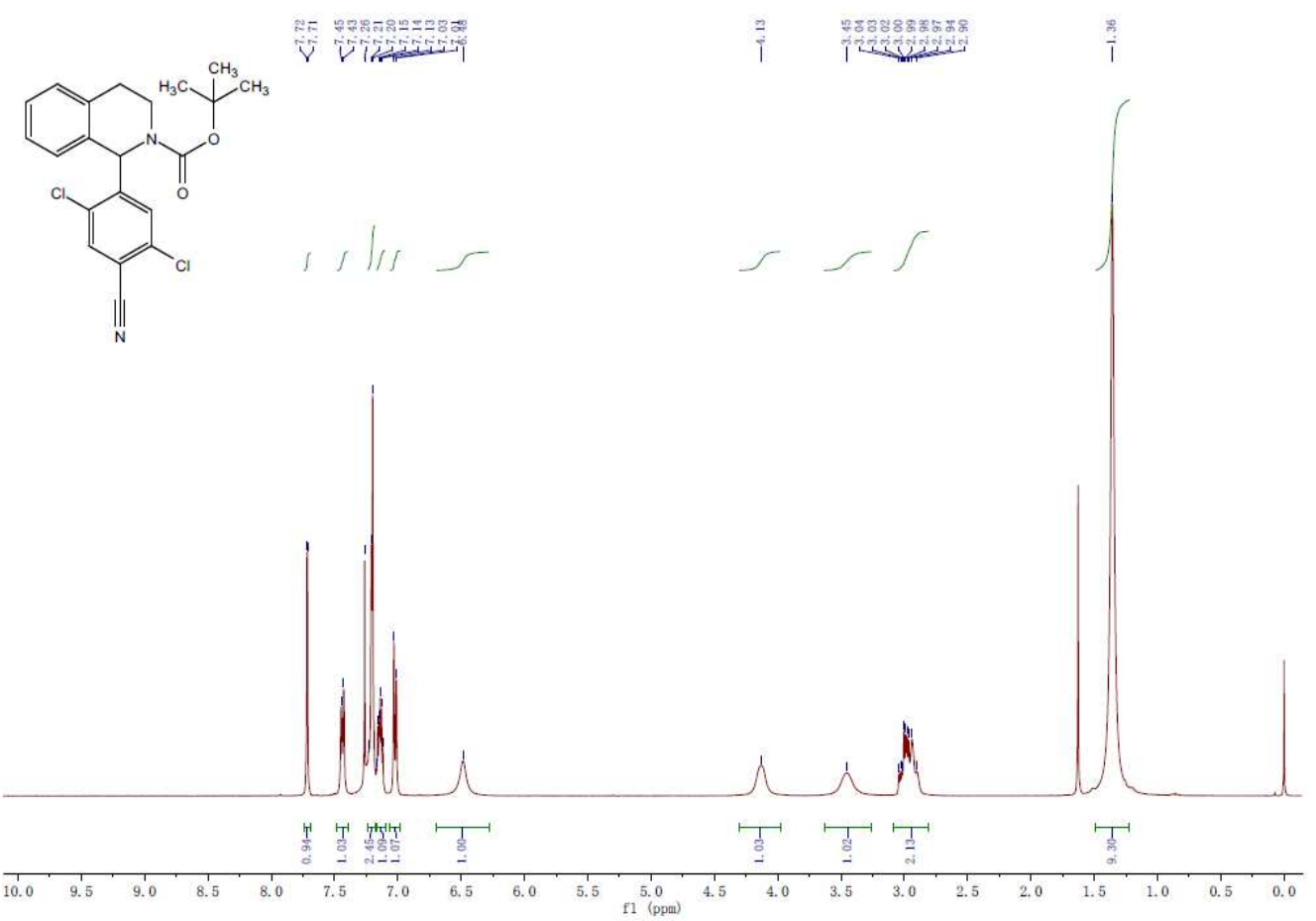

${ }^{13} \mathrm{C}$ NMR spectrum of compound 3ae.
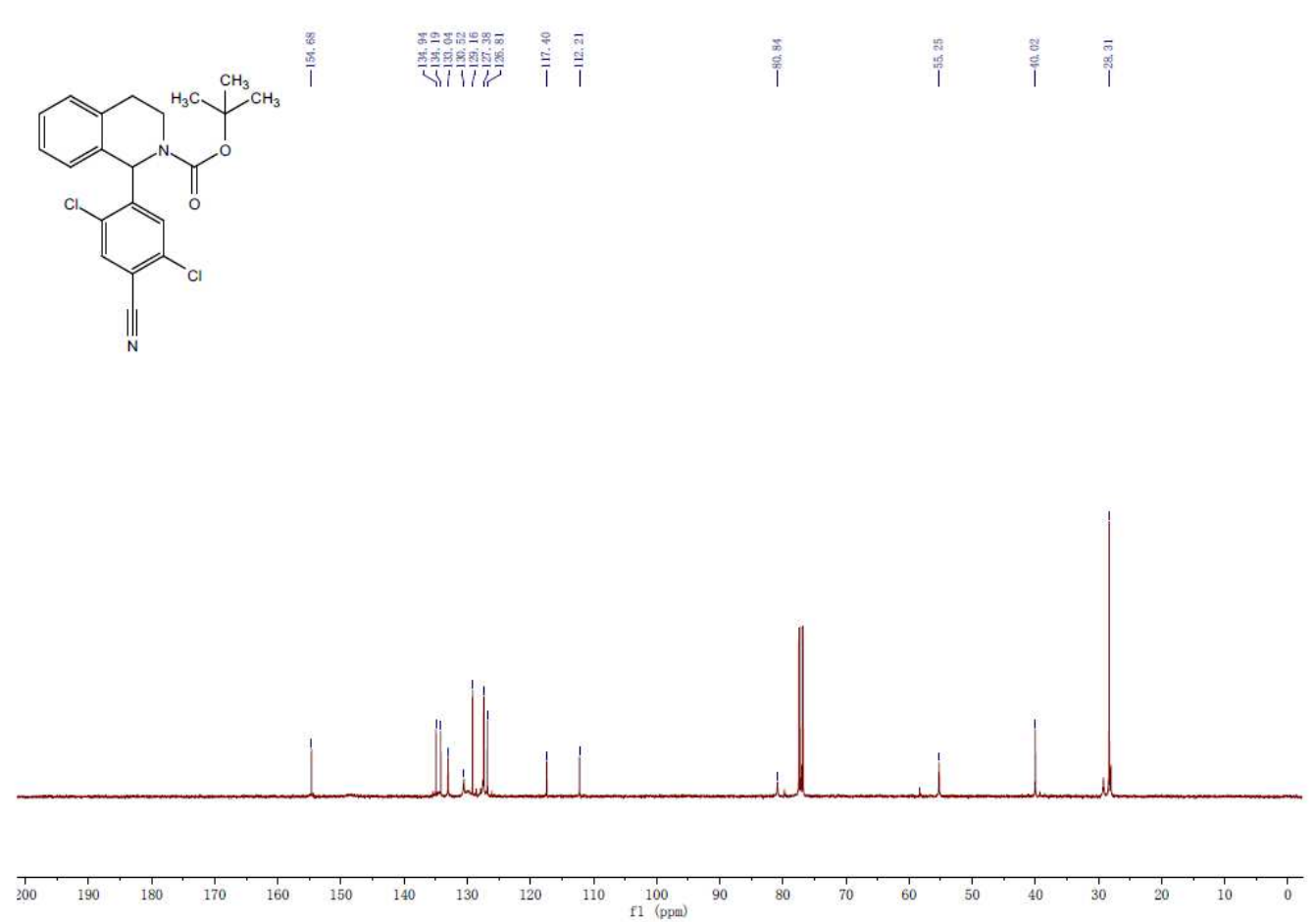
${ }^{1} \mathrm{H}$ NMR spectrum of compound $\mathbf{3 a f}$.
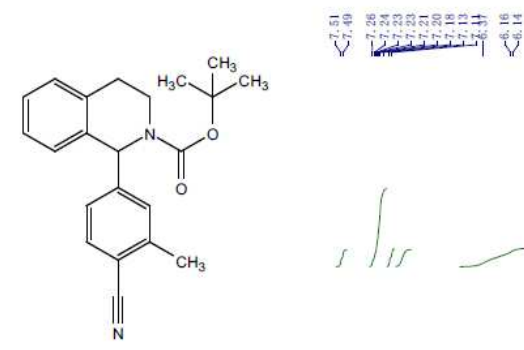

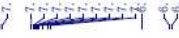
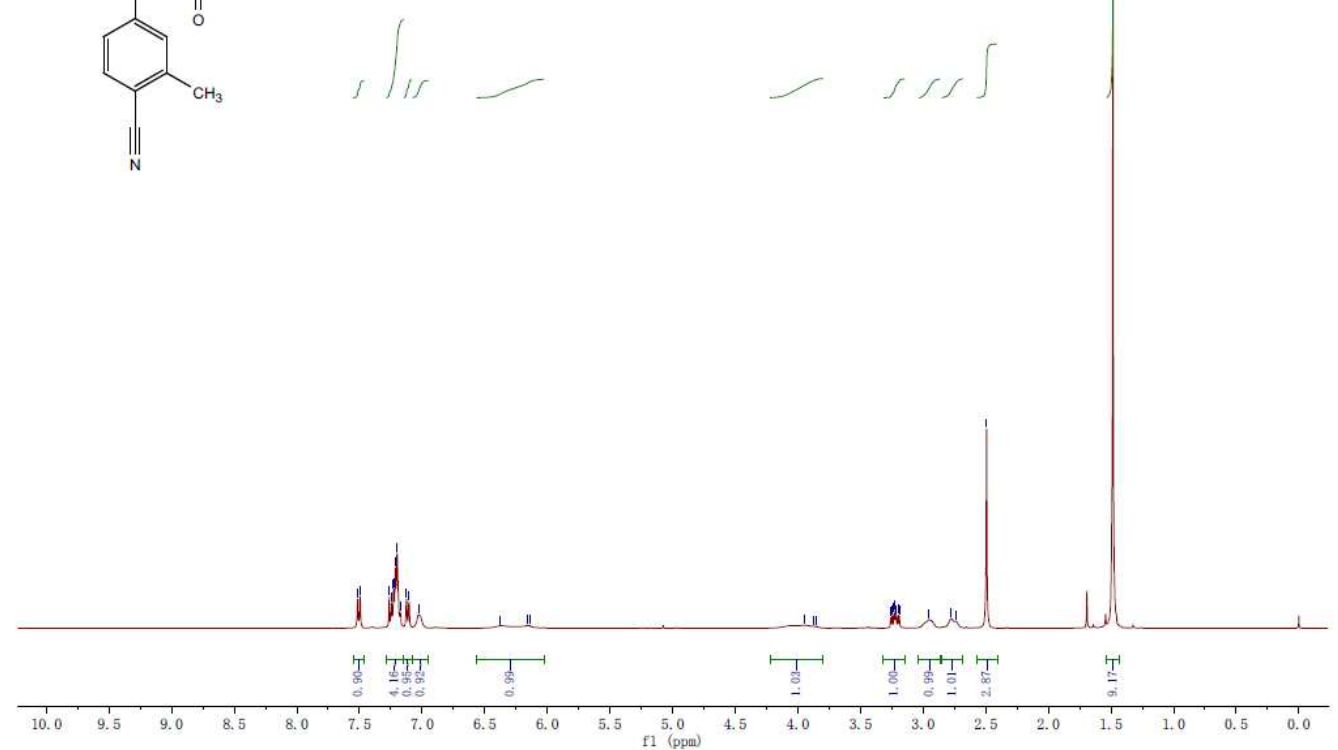

\section{${ }^{13} \mathrm{C}$ NMR spectrum of compound 3af.}
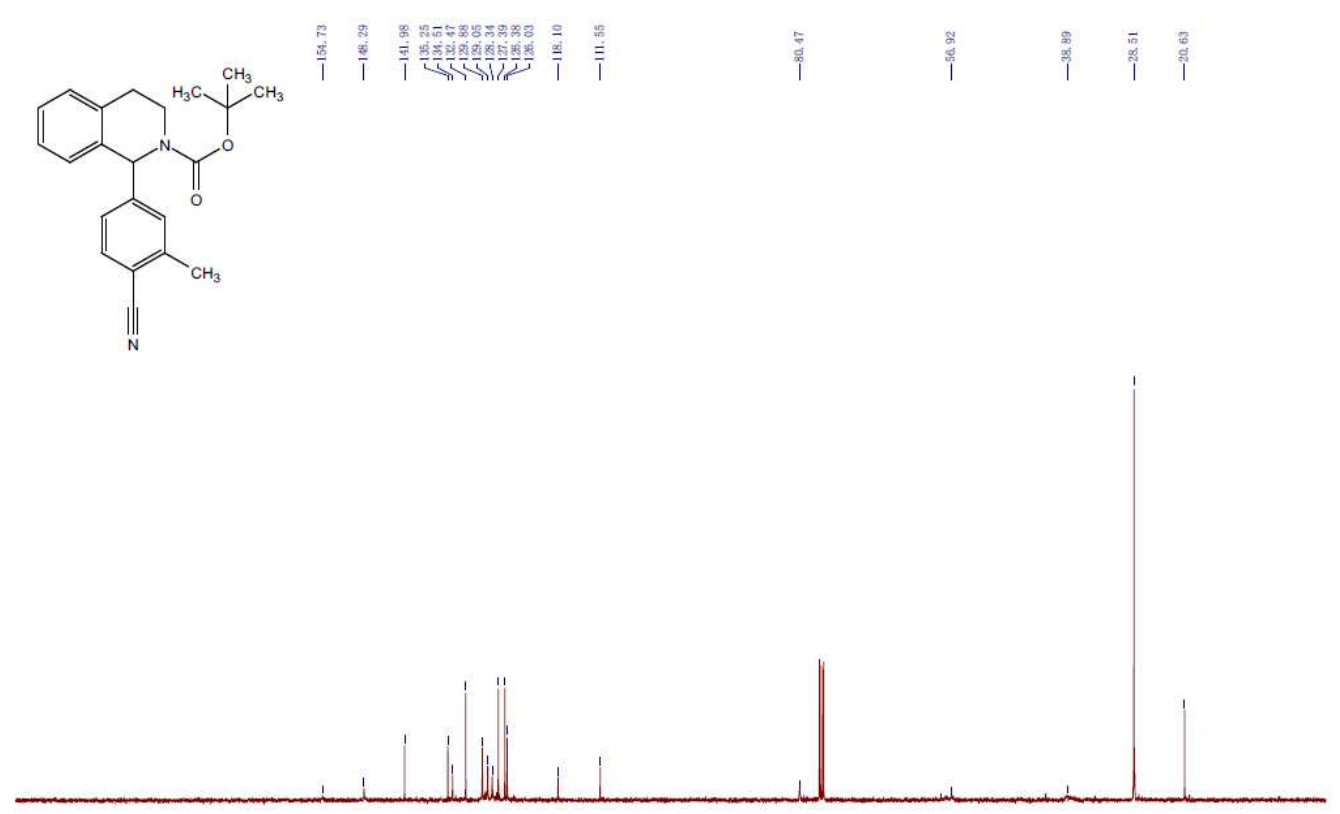

T⿱1 200

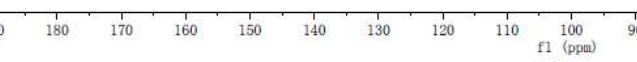


${ }^{1} \mathrm{H}$ NMR spectrum of compound 3ag.

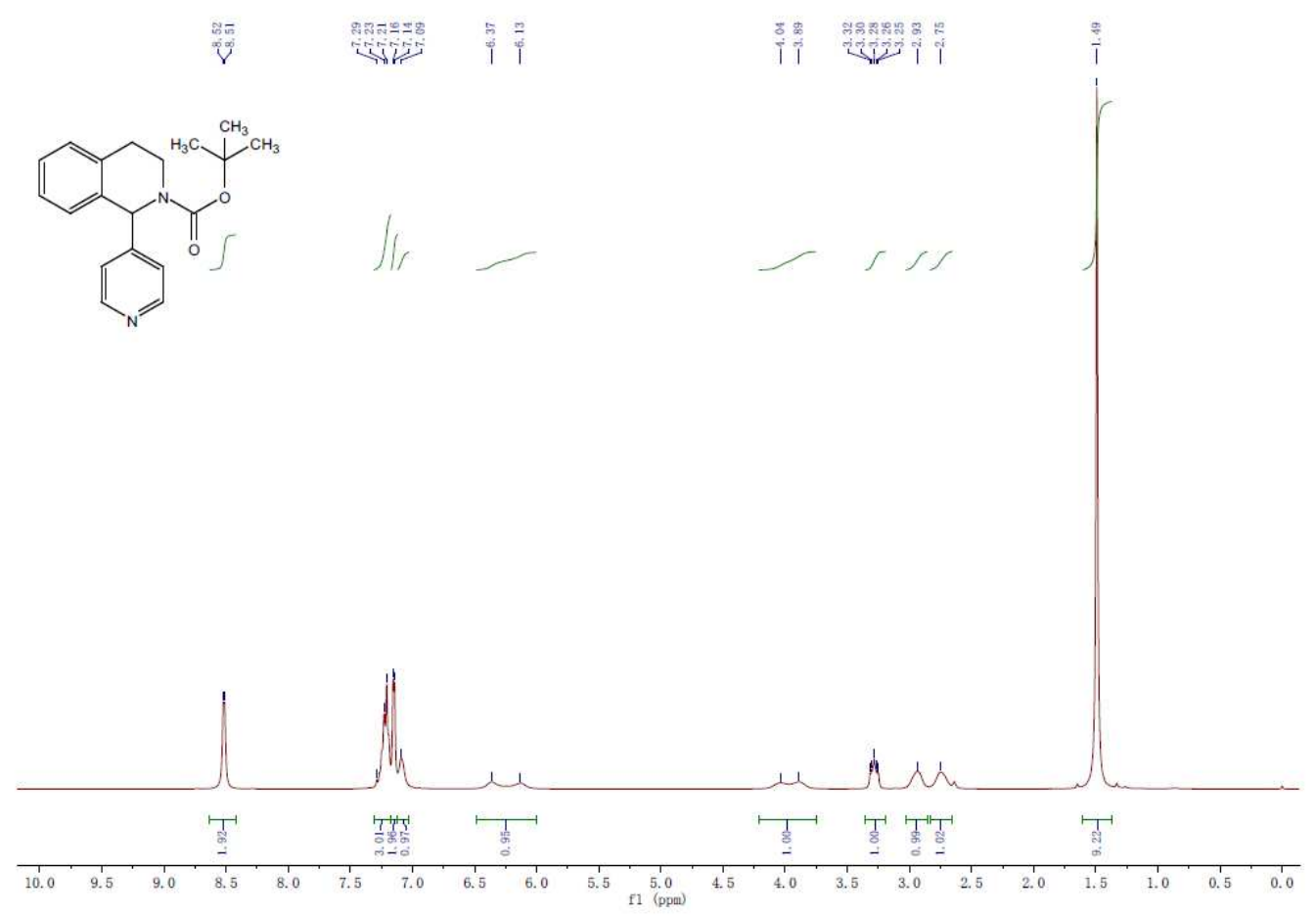

${ }^{13} \mathrm{C}$ NMR spectrum of compound 3ag.
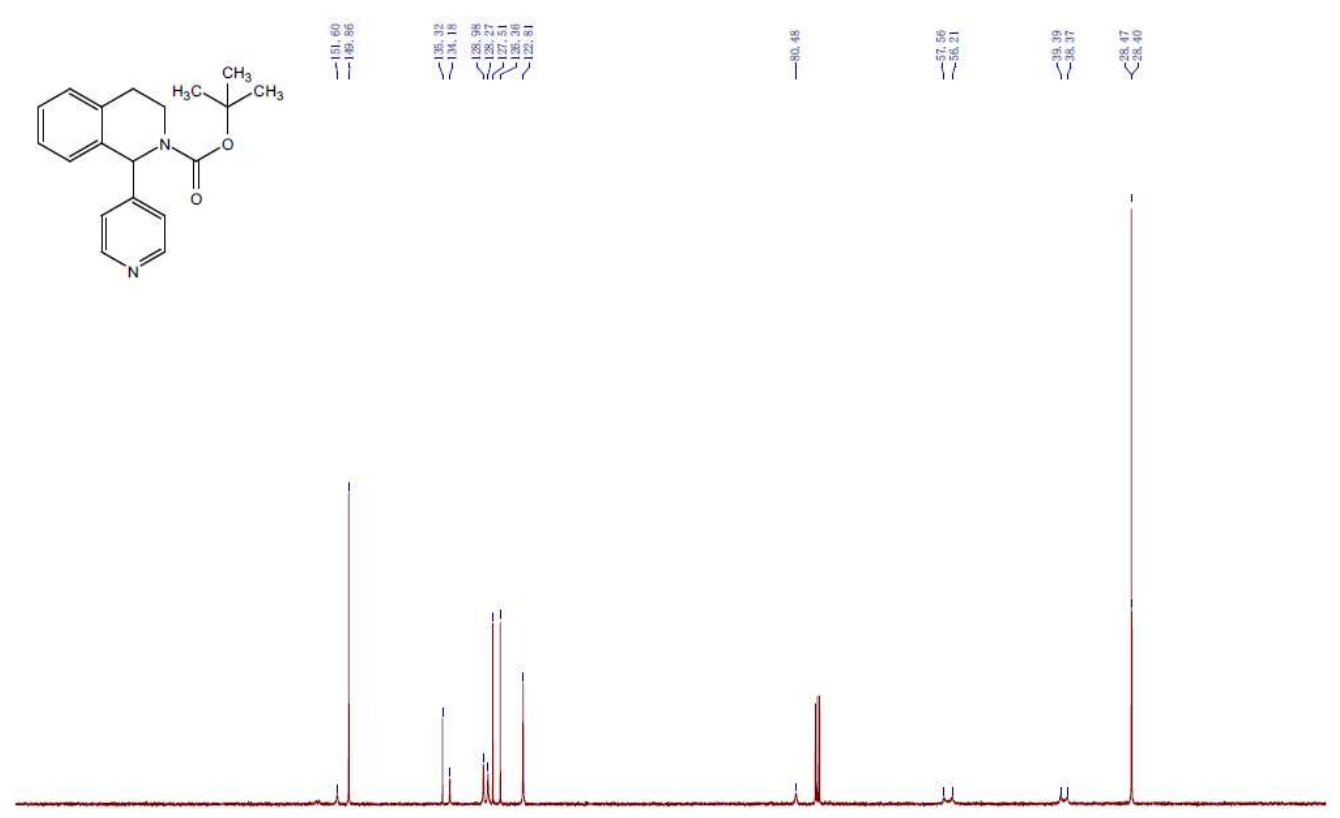

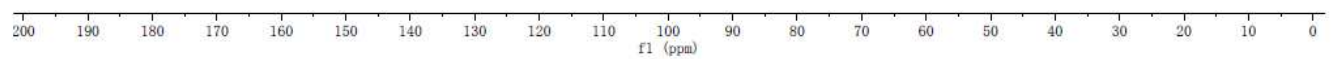


${ }^{1} \mathrm{H}$ NMR spectrum of compound $\mathbf{3 a h}$.

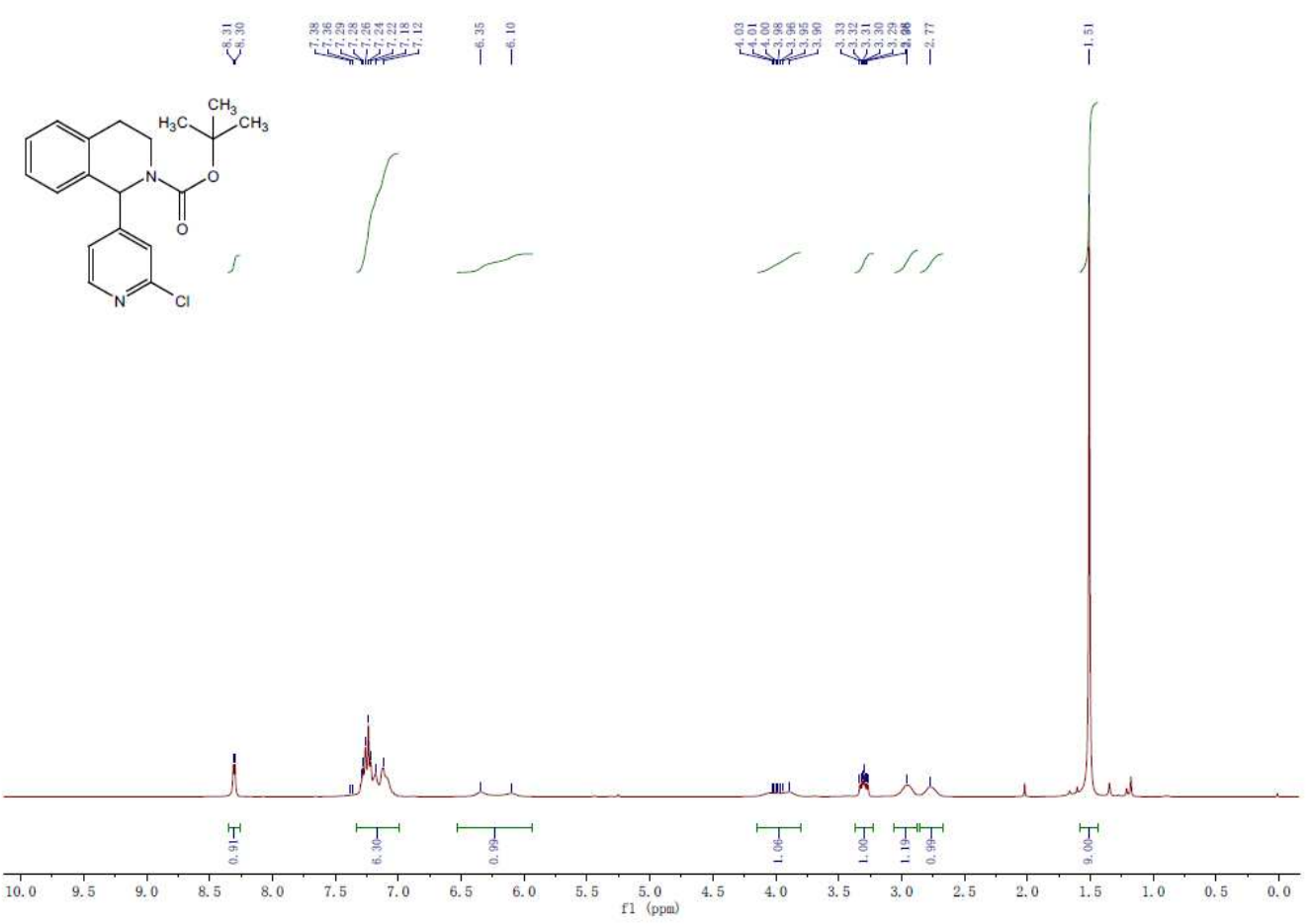

${ }^{13} \mathrm{C}$ NMR spectrum of compound $\mathbf{3 a h}$.
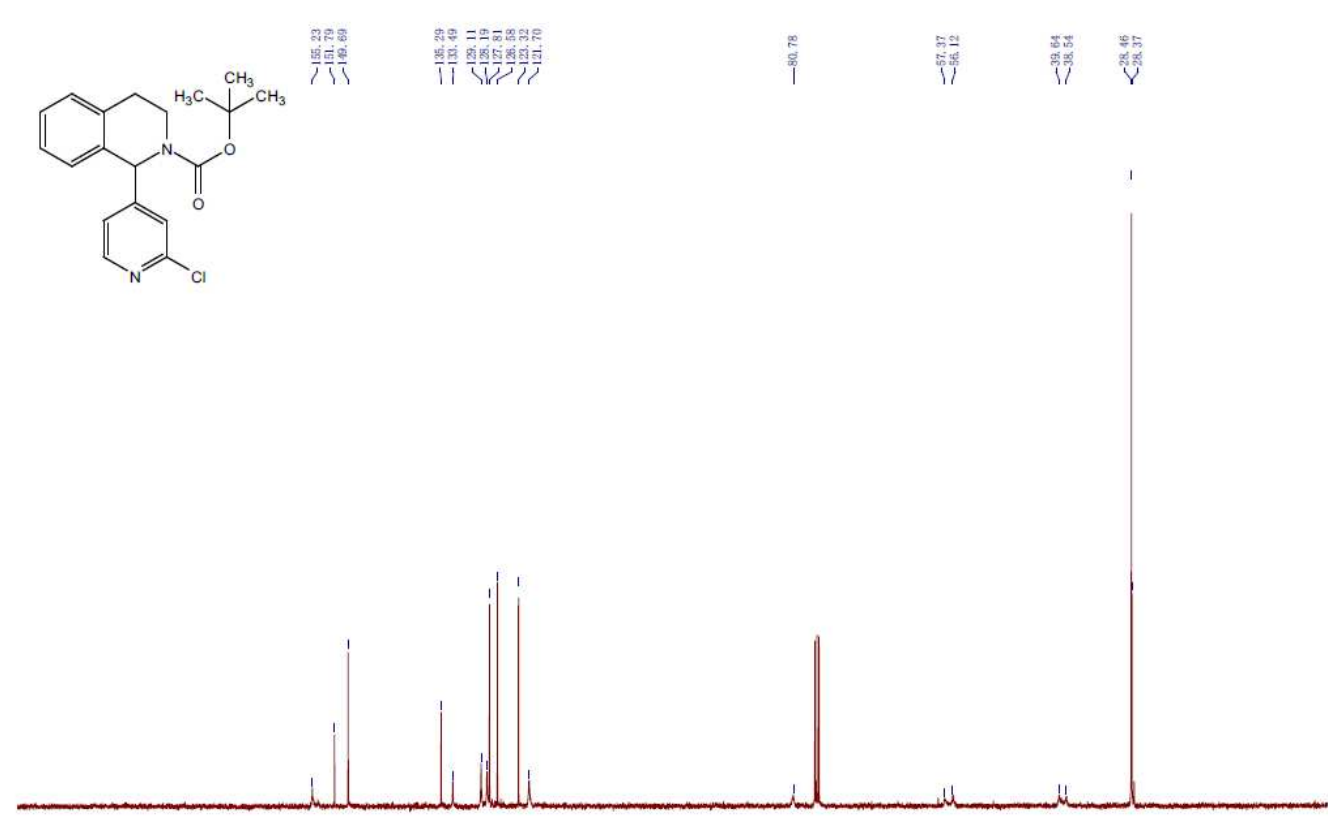

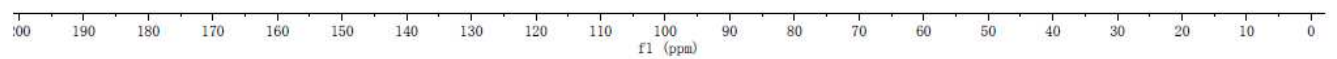


${ }^{1} \mathrm{H}$ NMR spectrum of compound 3ai.
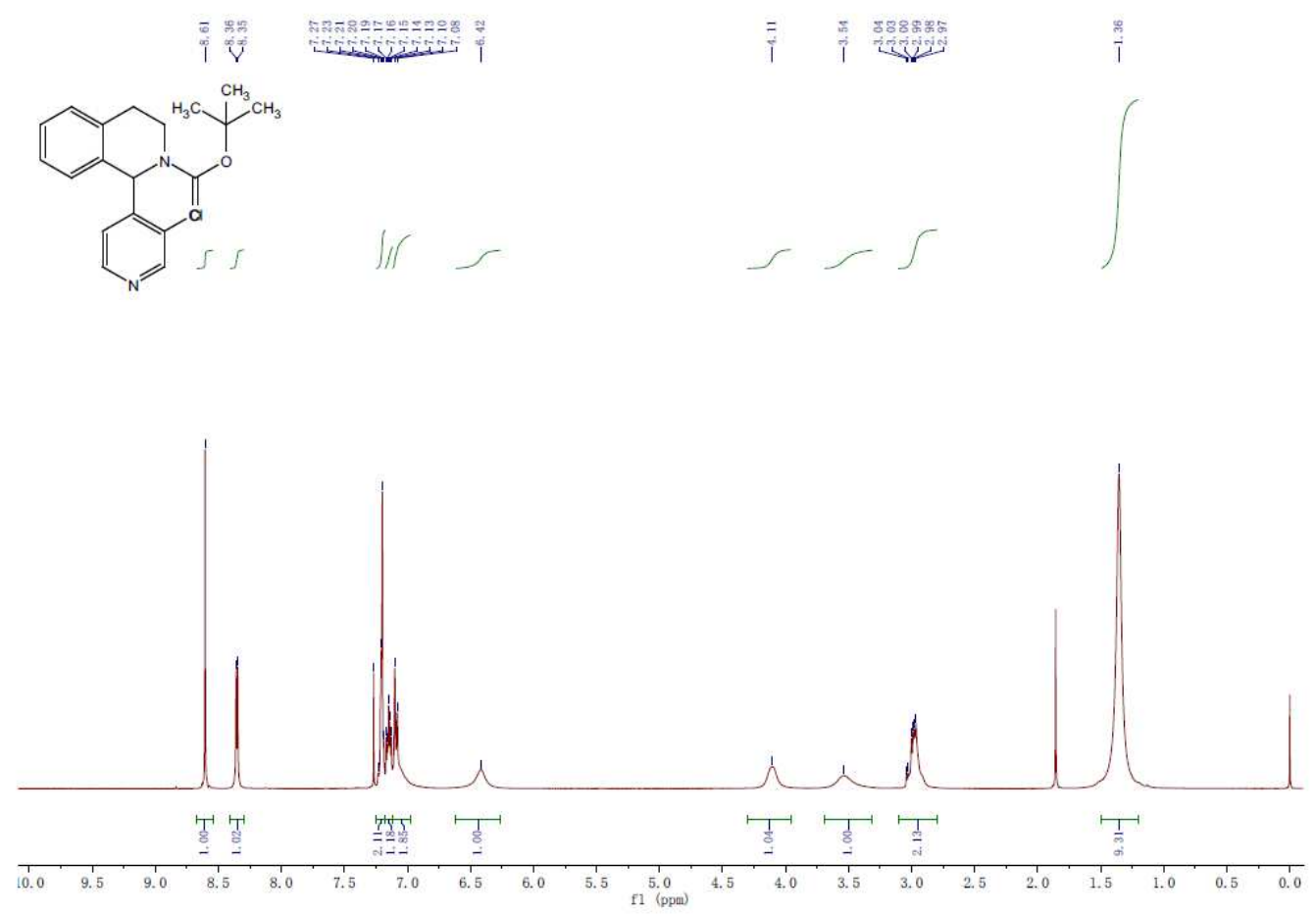

${ }^{13} \mathrm{C}$ NMR spectrum of compound 3ai.
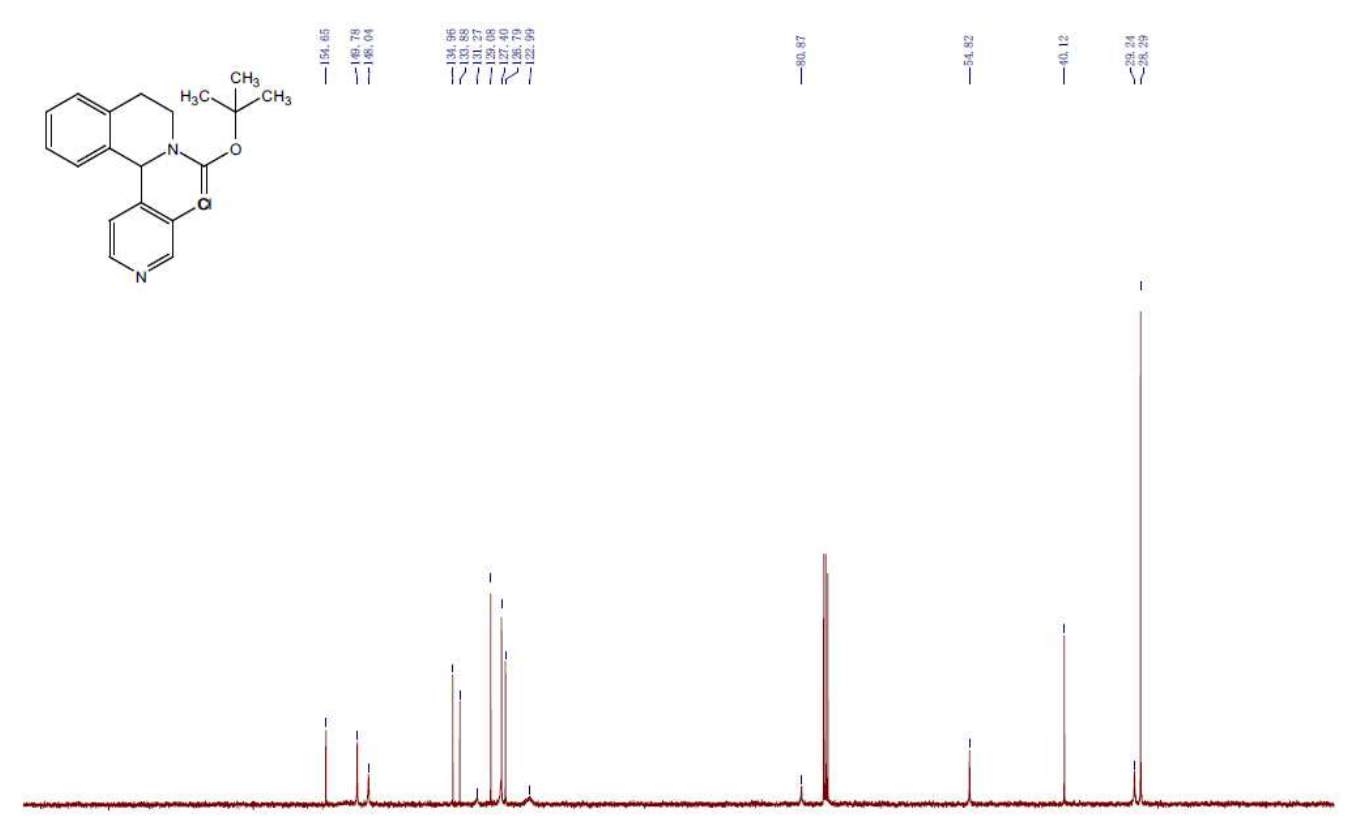

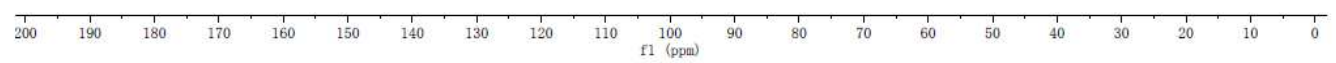


${ }^{1}$ H NMR spectrum of compound 3aj.

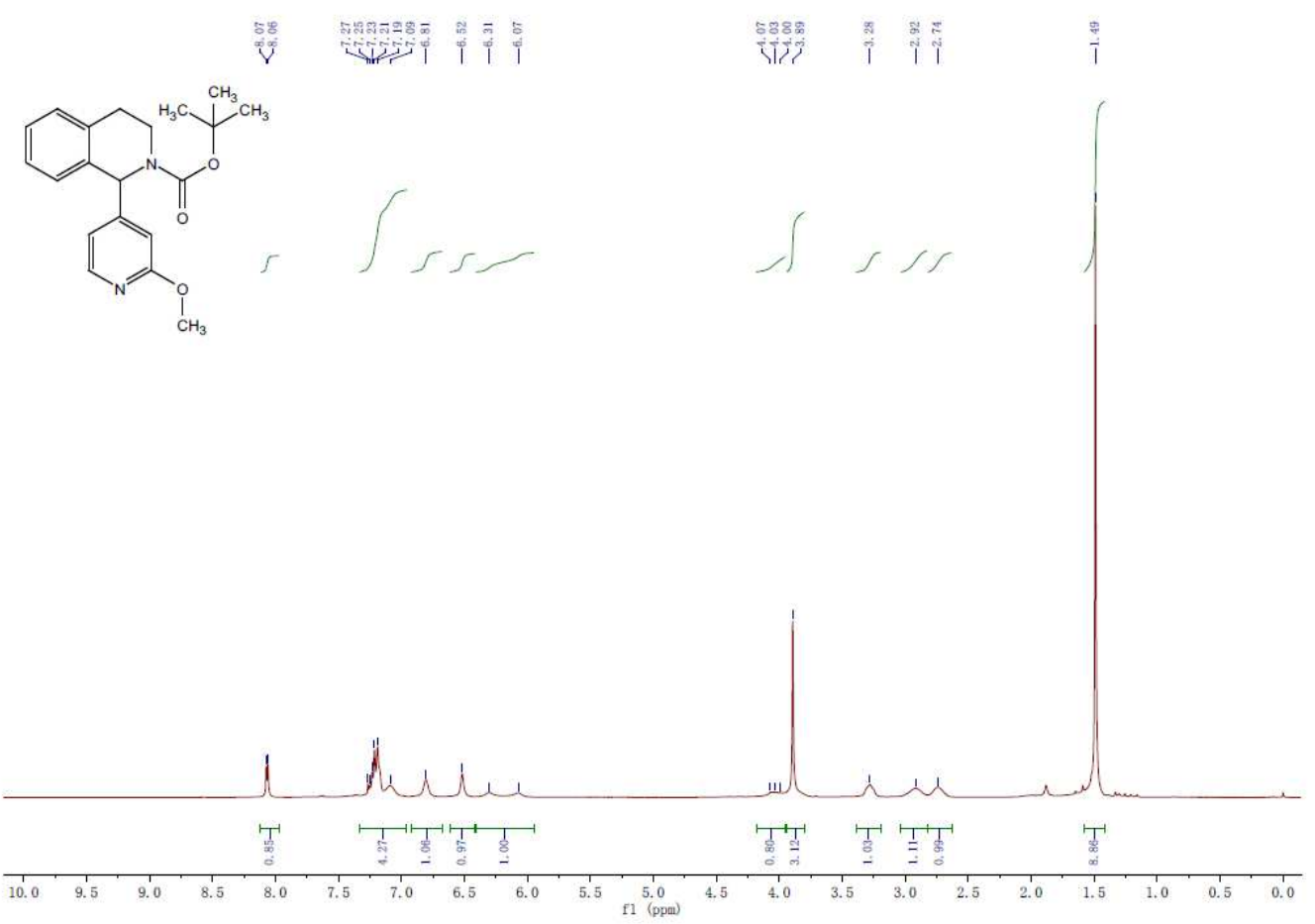

${ }^{13} \mathrm{C}$ NMR spectrum of compound 3aj.
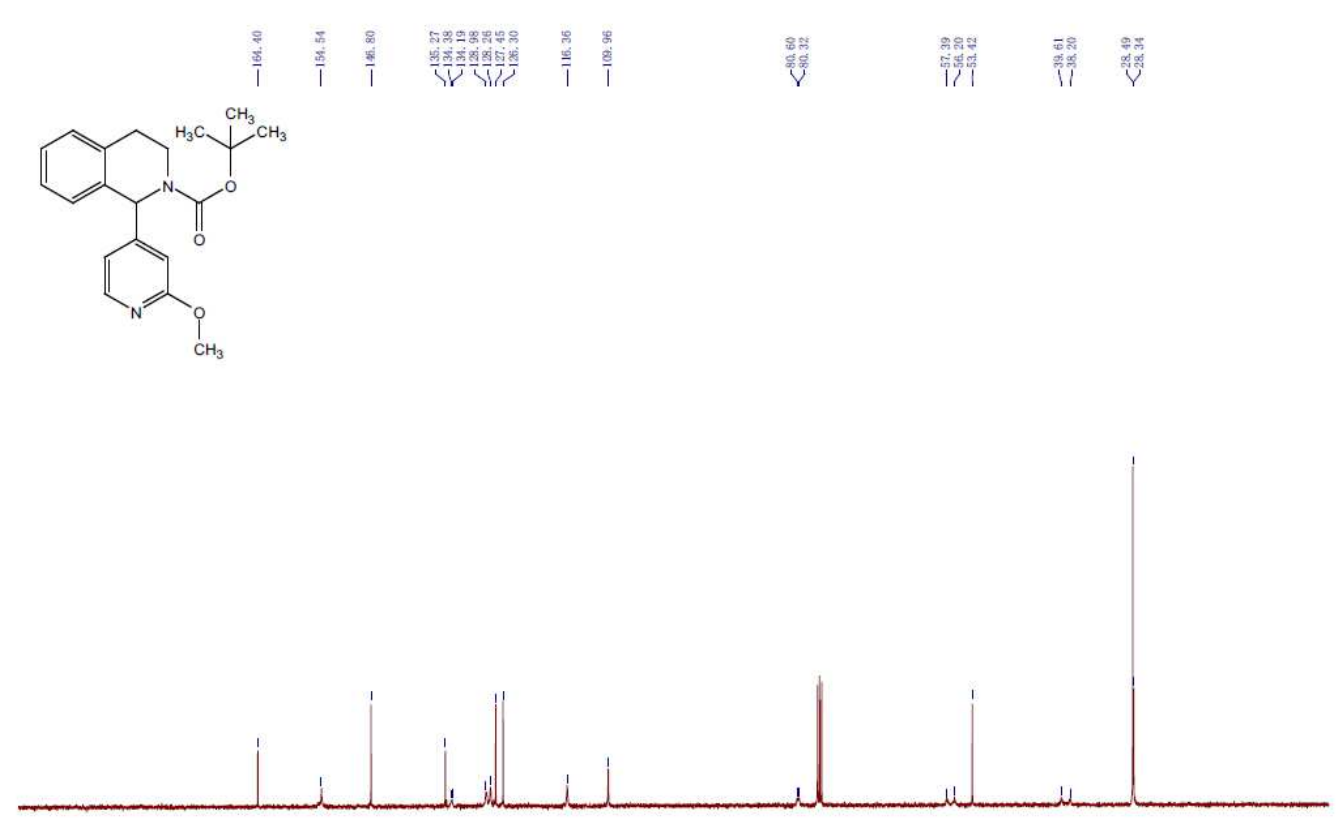

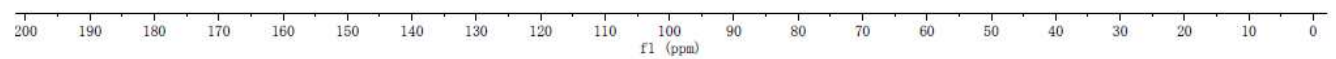


${ }^{1} \mathrm{H}$ NMR spectrum of compound $\mathbf{3 b b}$.

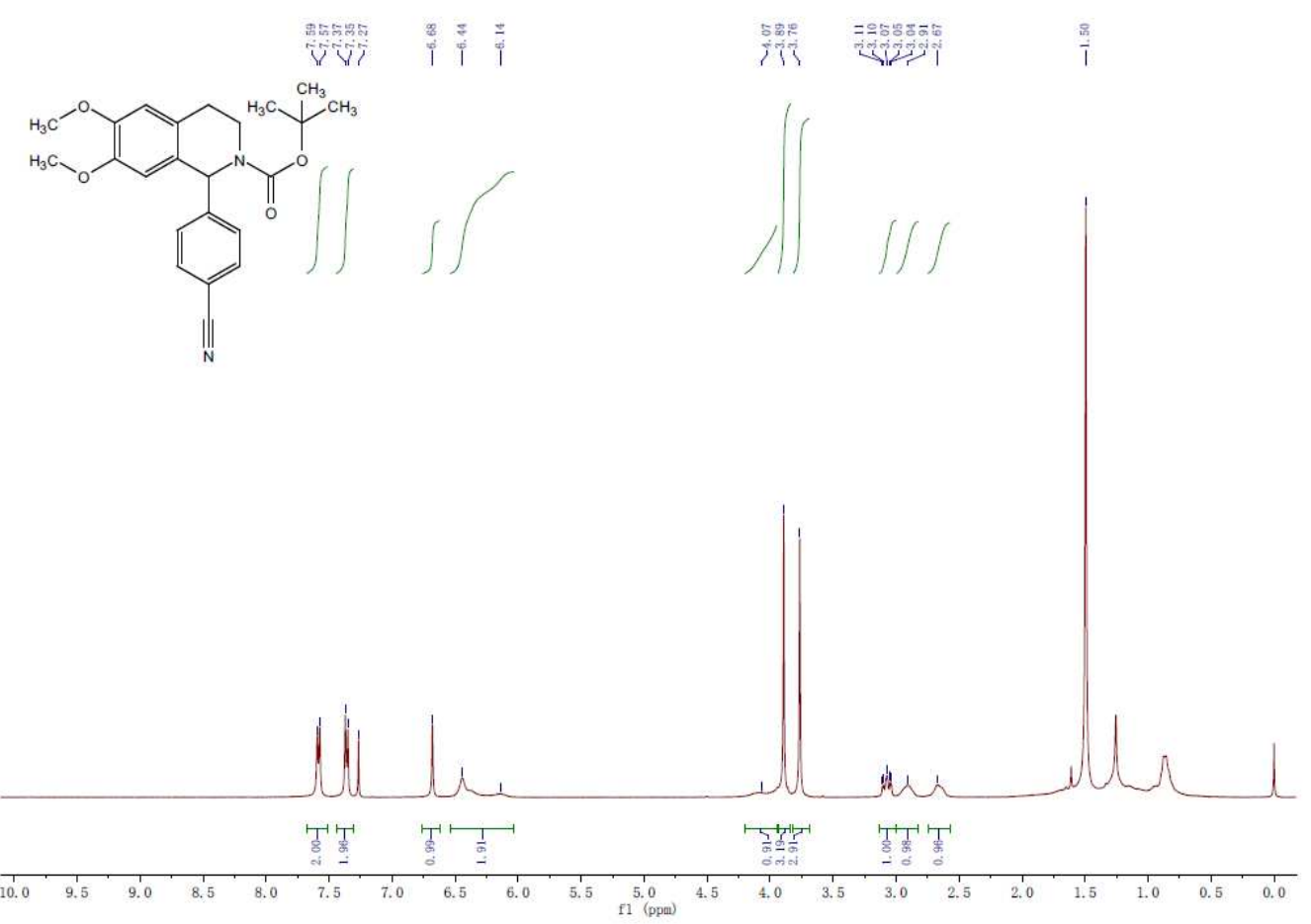

${ }^{13} \mathrm{C}$ NMR spectrum of compound $\mathbf{3 b b}$.
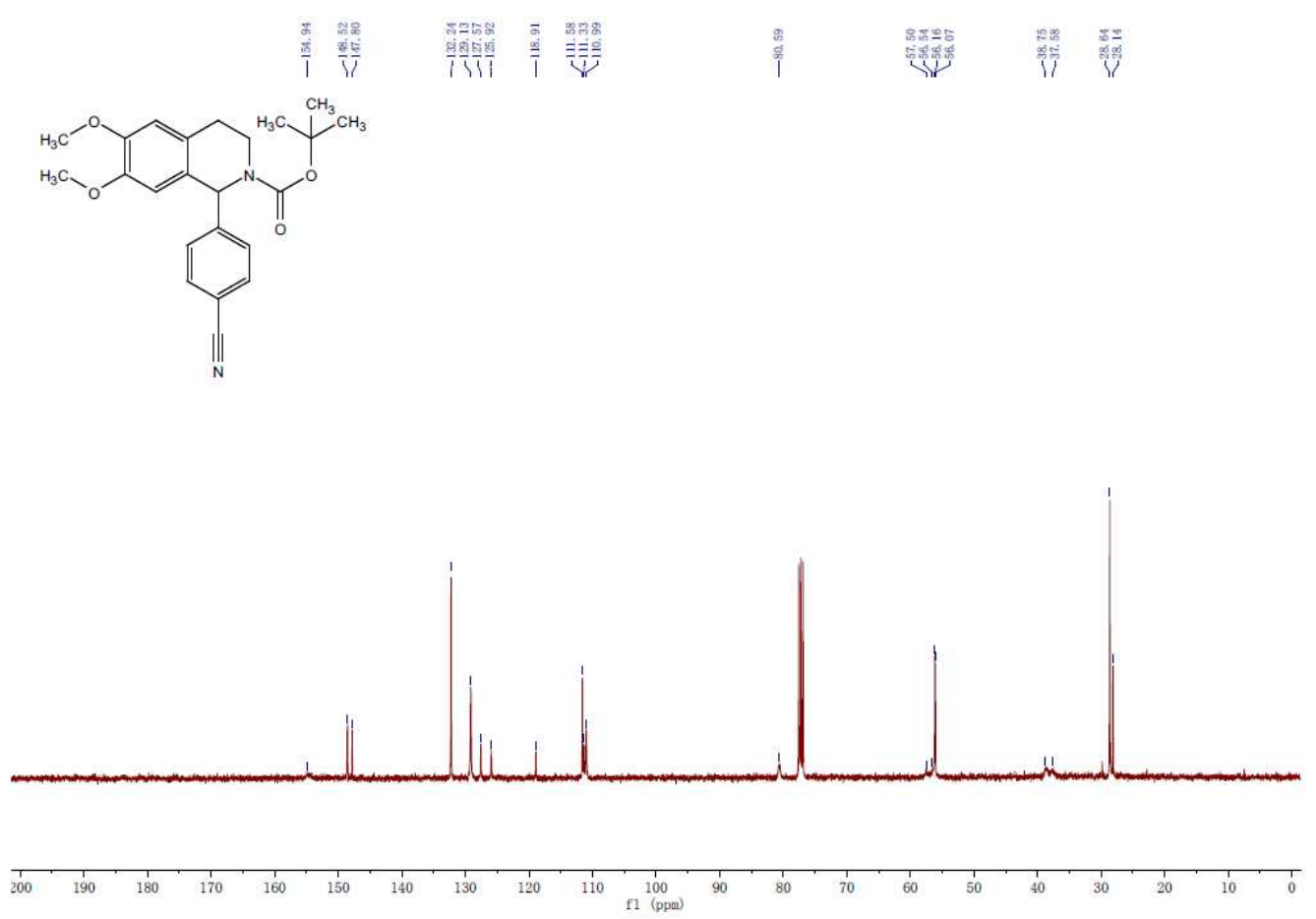

S32 
${ }^{1}$ H NMR spectrum of compound $\mathbf{3 c b}$.

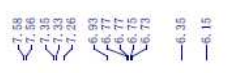

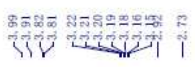

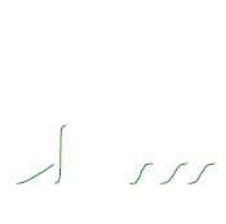

$\stackrel{-\infty}{10}$
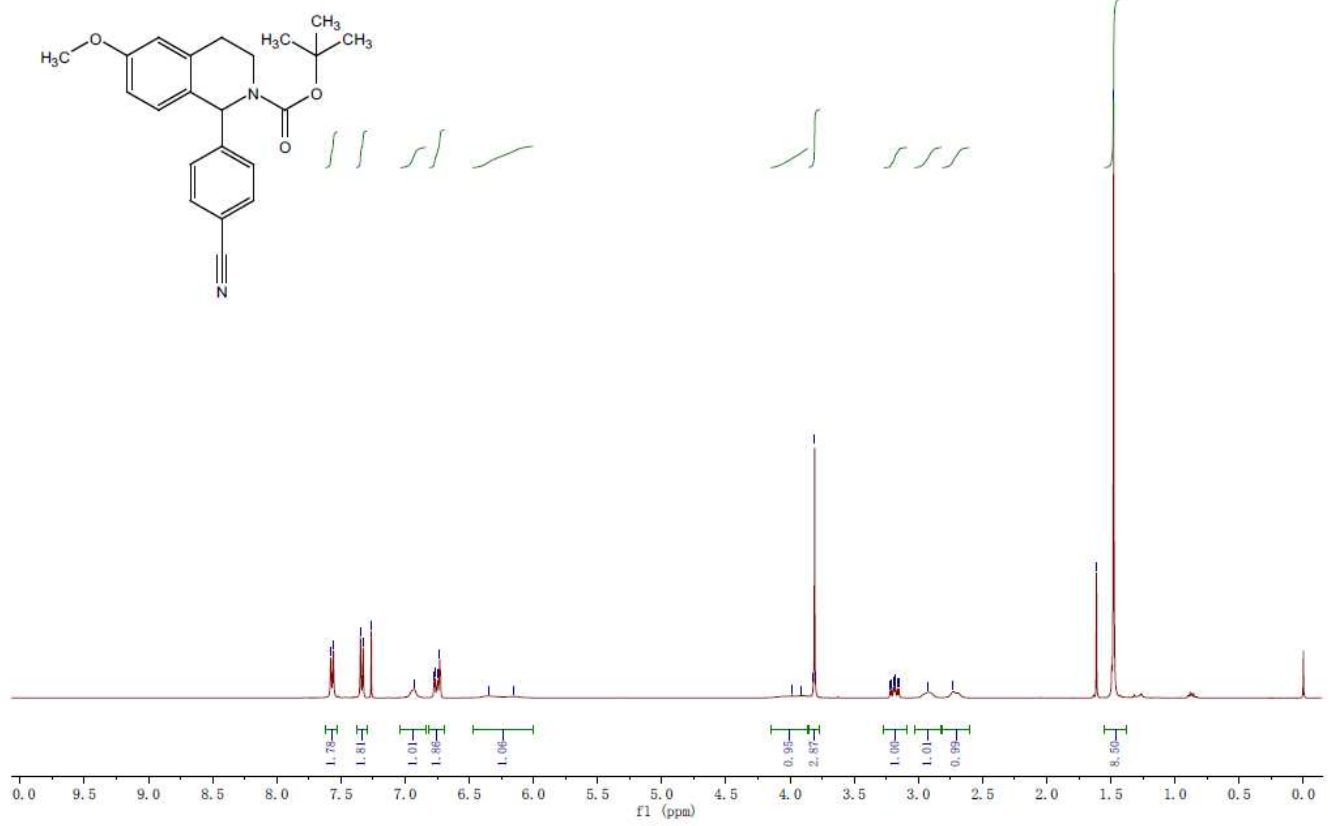

${ }^{13} \mathrm{C}$ NMR spectrum of compound $\mathbf{3} \mathbf{c b}$.
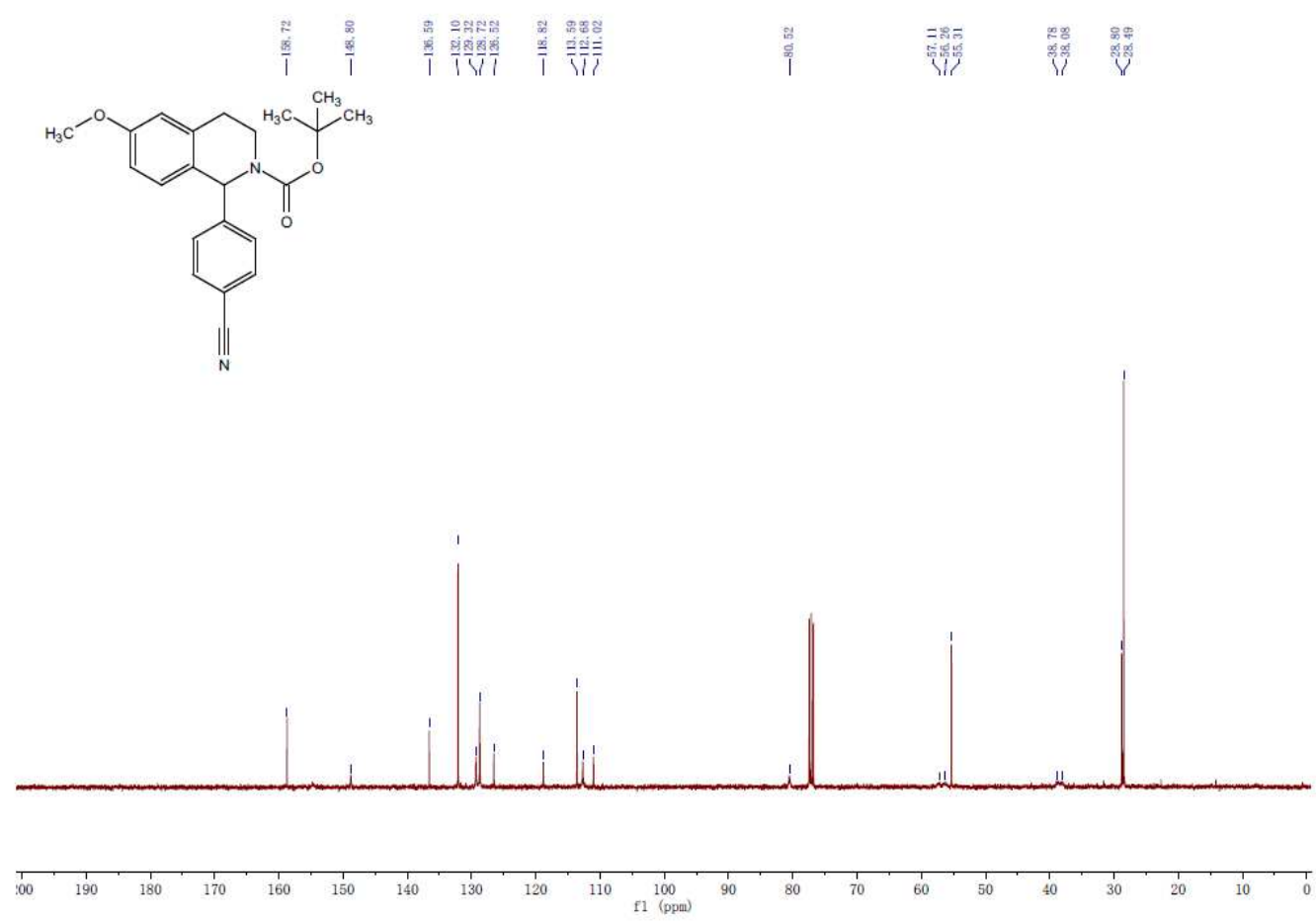

S33 
${ }^{1} \mathrm{H}$ NMR spectrum of compound $\mathbf{3 d b}$.

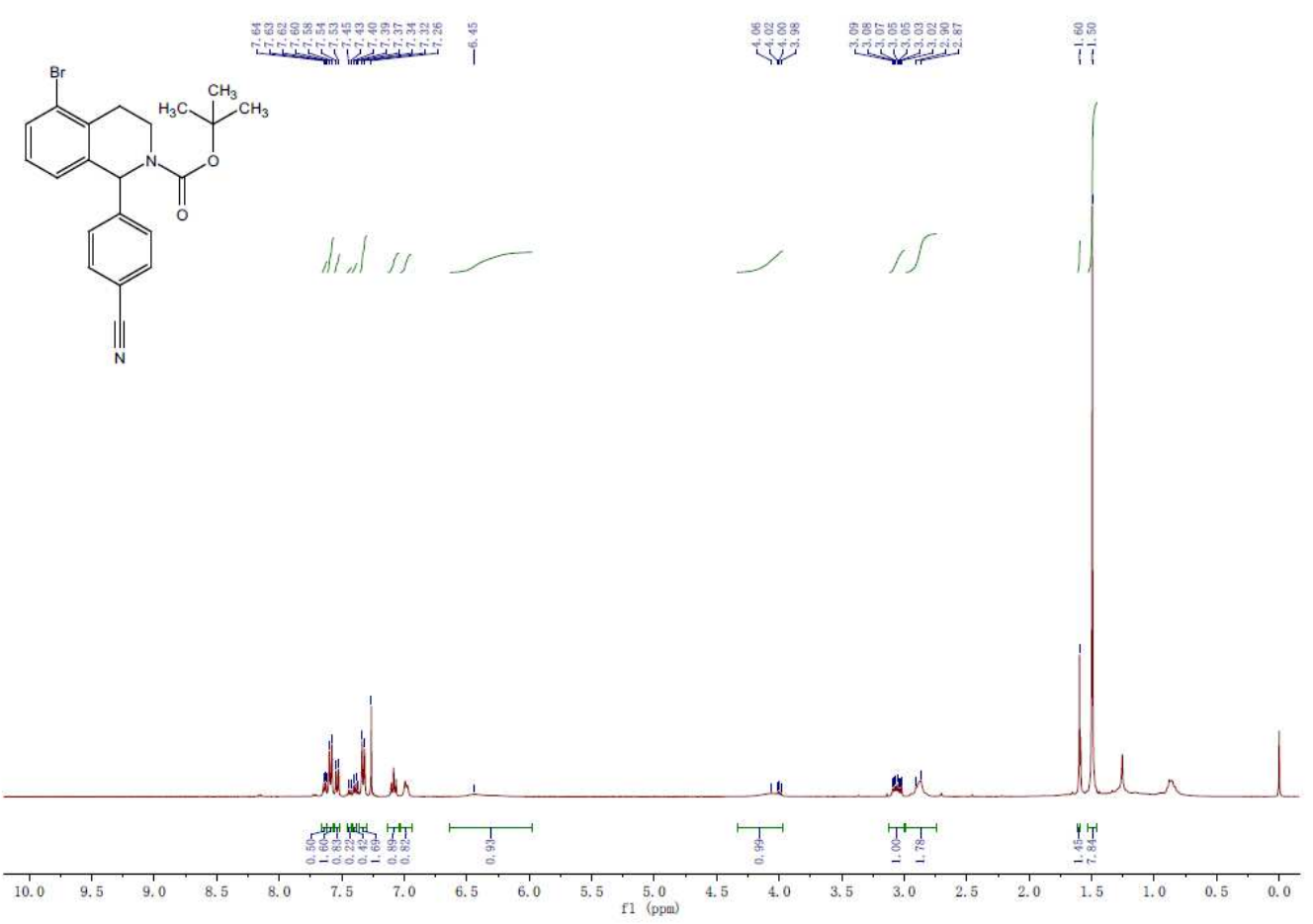

${ }^{13} \mathrm{C}$ NMR spectrum of compound $\mathbf{3 d b}$.
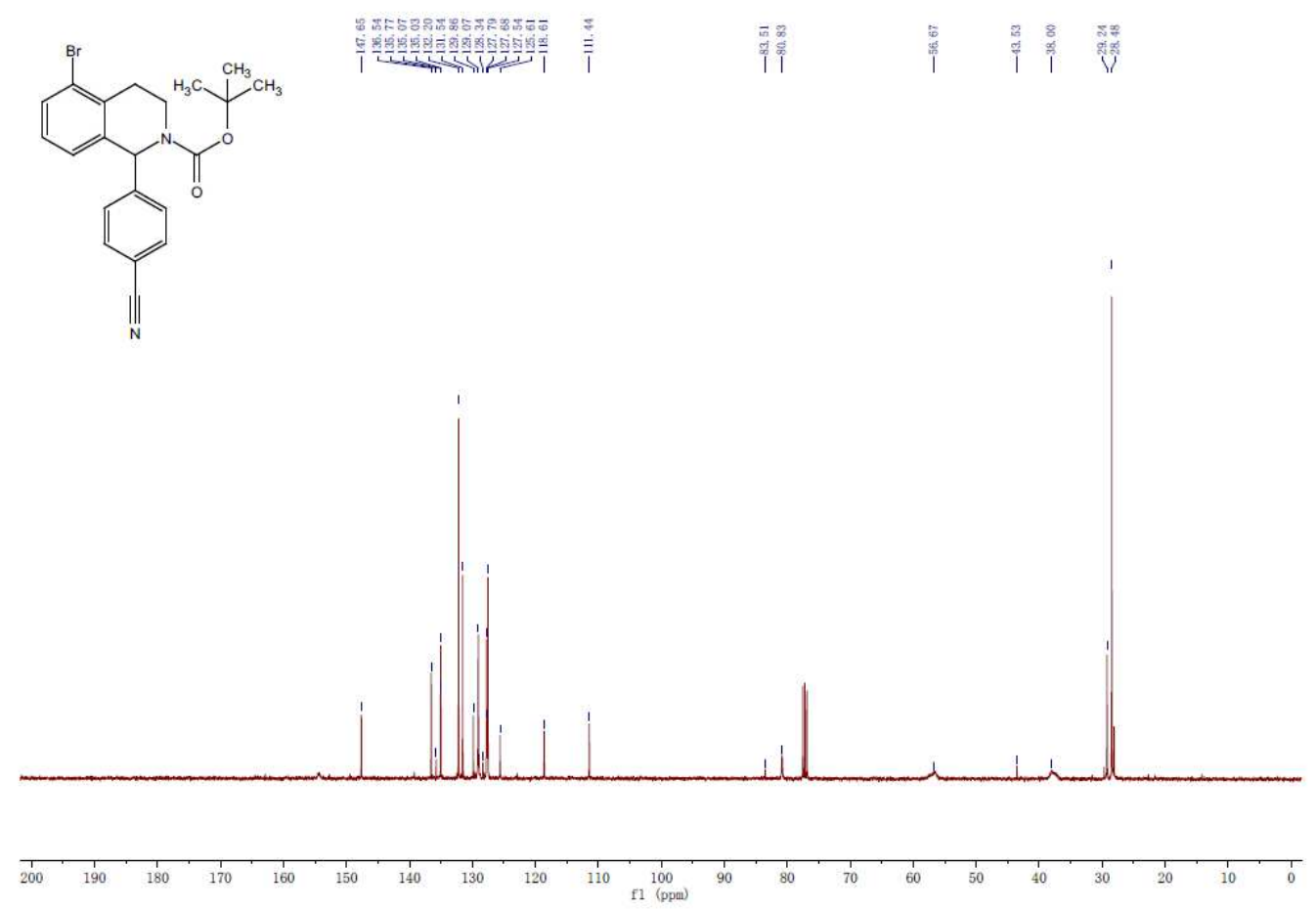
${ }^{1} \mathrm{H}$ NMR spectrum of compound $\mathbf{3 e b}$.

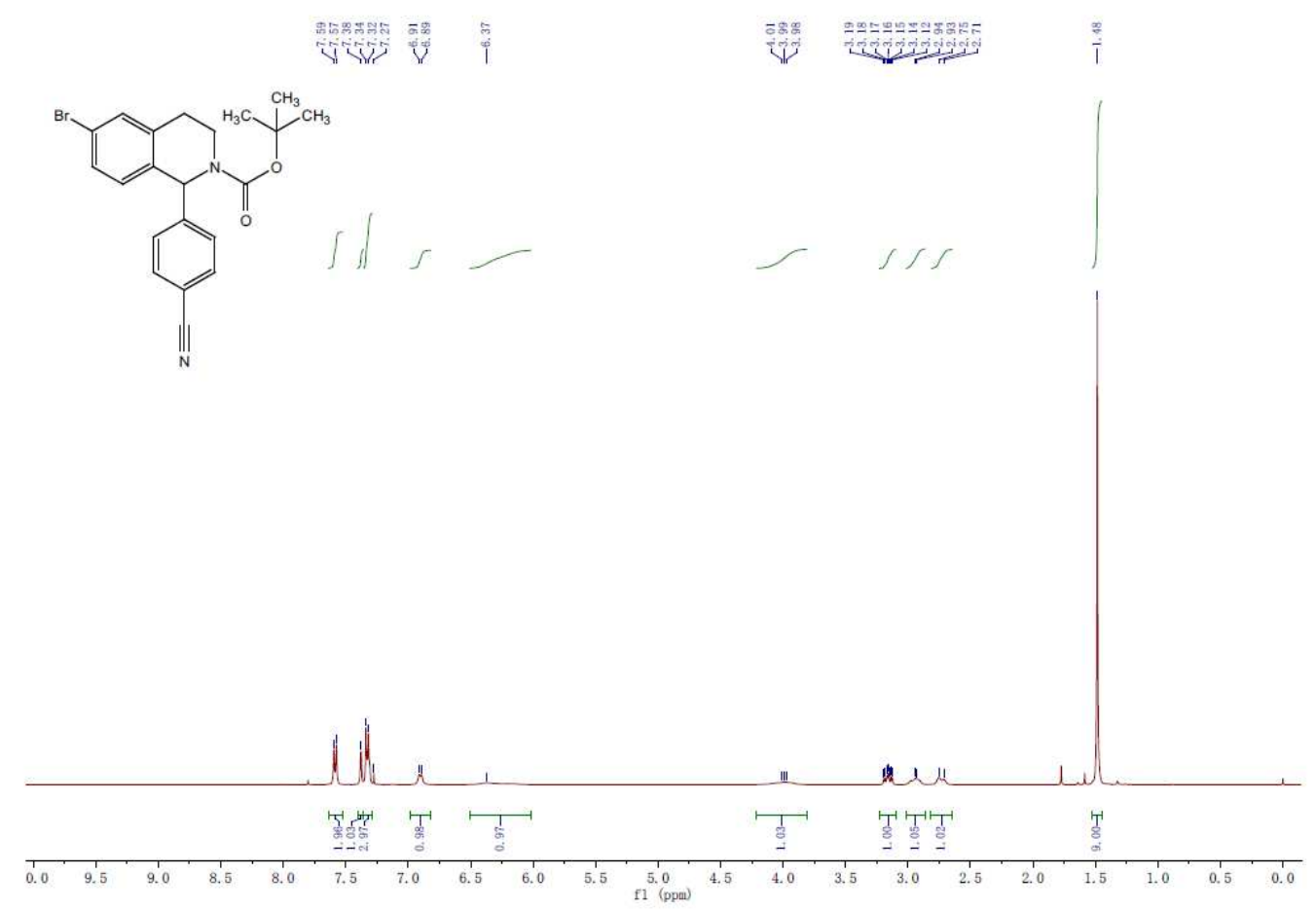

${ }^{13} \mathrm{C}$ NMR spectrum of compound $\mathbf{3 e b}$.

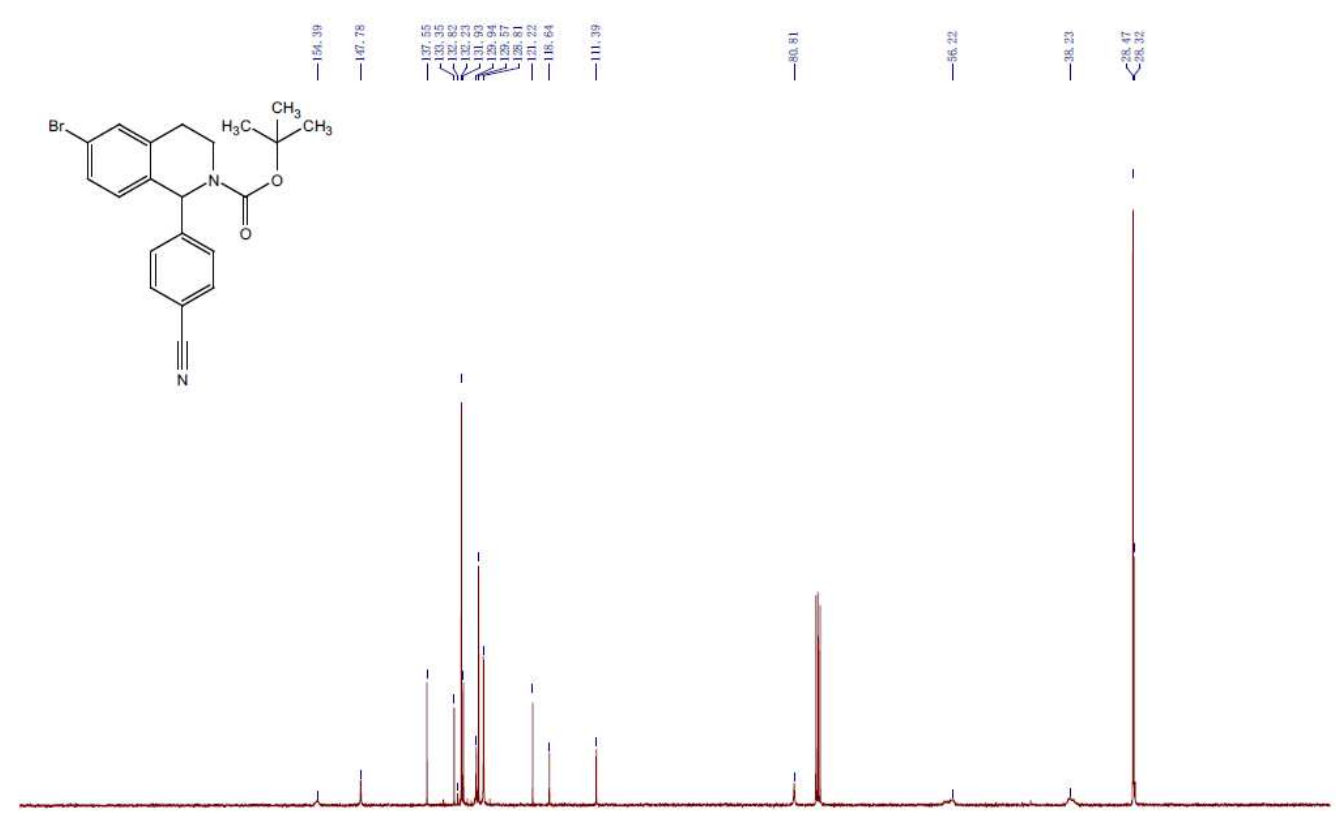

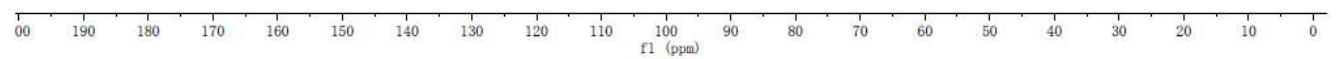


${ }^{1}$ H NMR spectrum of compound $\mathbf{3} \mathbf{f b}$.

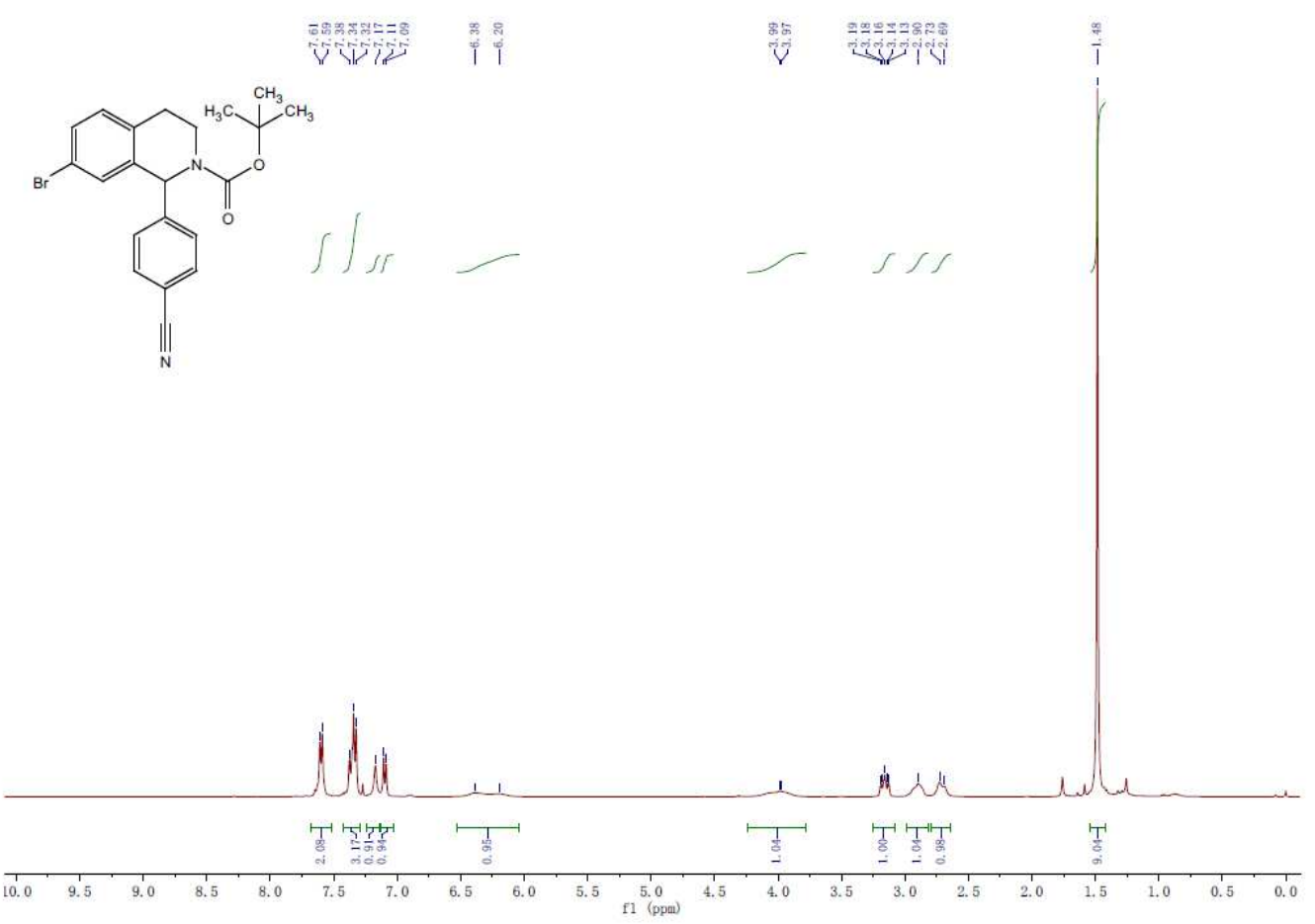

${ }^{13} \mathrm{C}$ NMR spectrum of compound $\mathbf{3} \mathbf{f b}$.
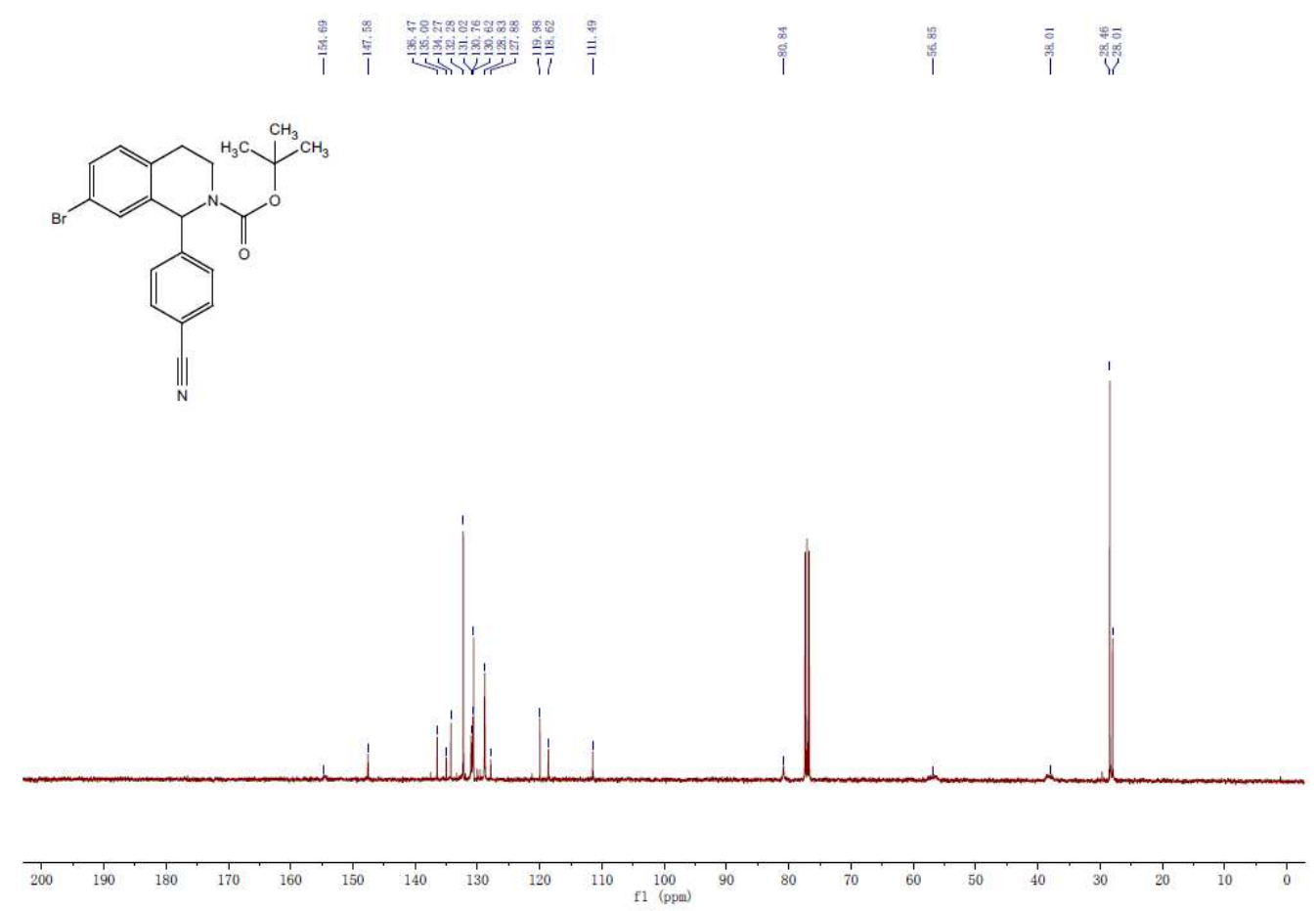
${ }^{1} \mathrm{H}$ NMR spectrum of compound $\mathbf{3 g b}$.

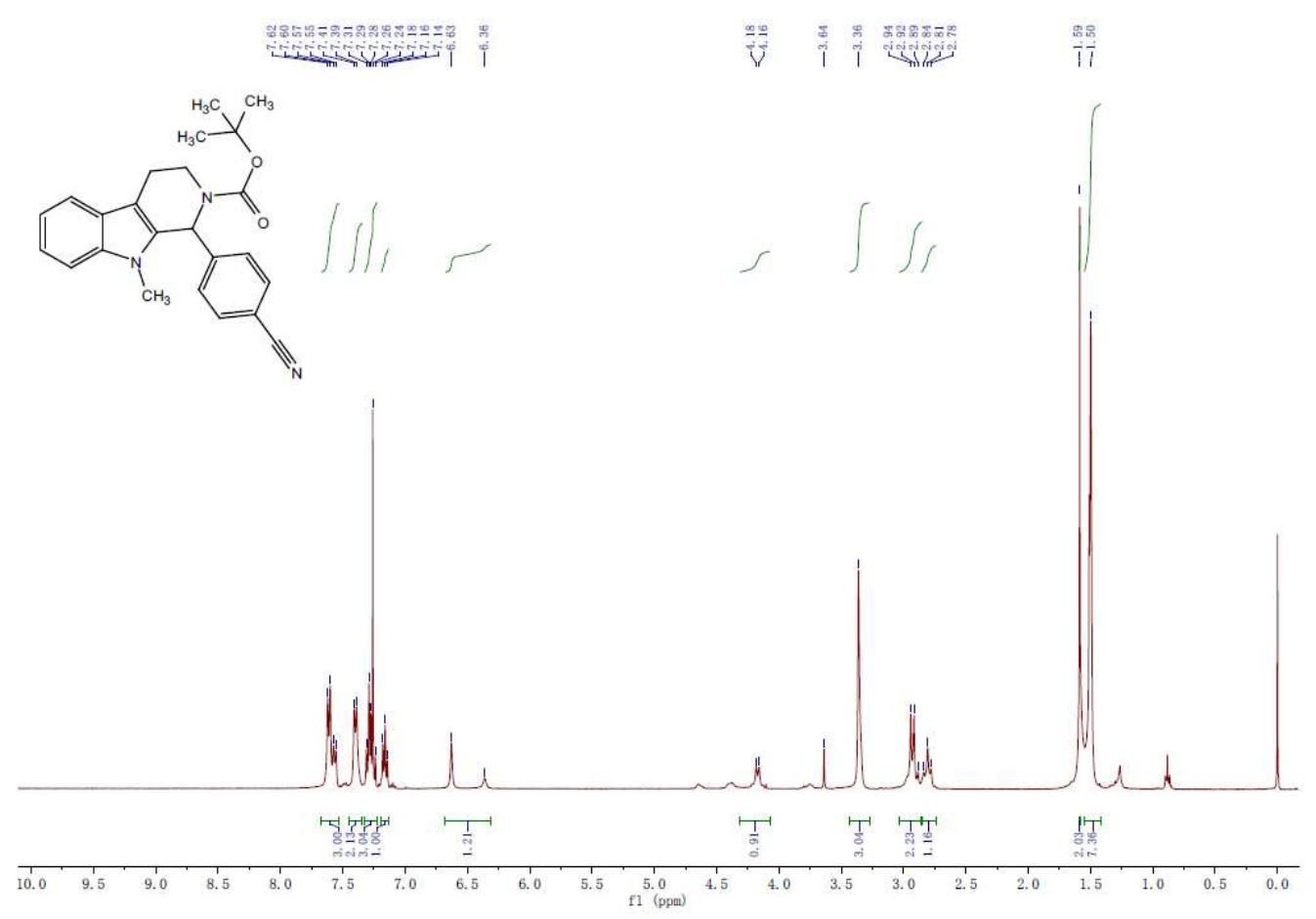

${ }^{13} \mathrm{C}$ NMR spectrum of compound $\mathbf{3 g b}$.
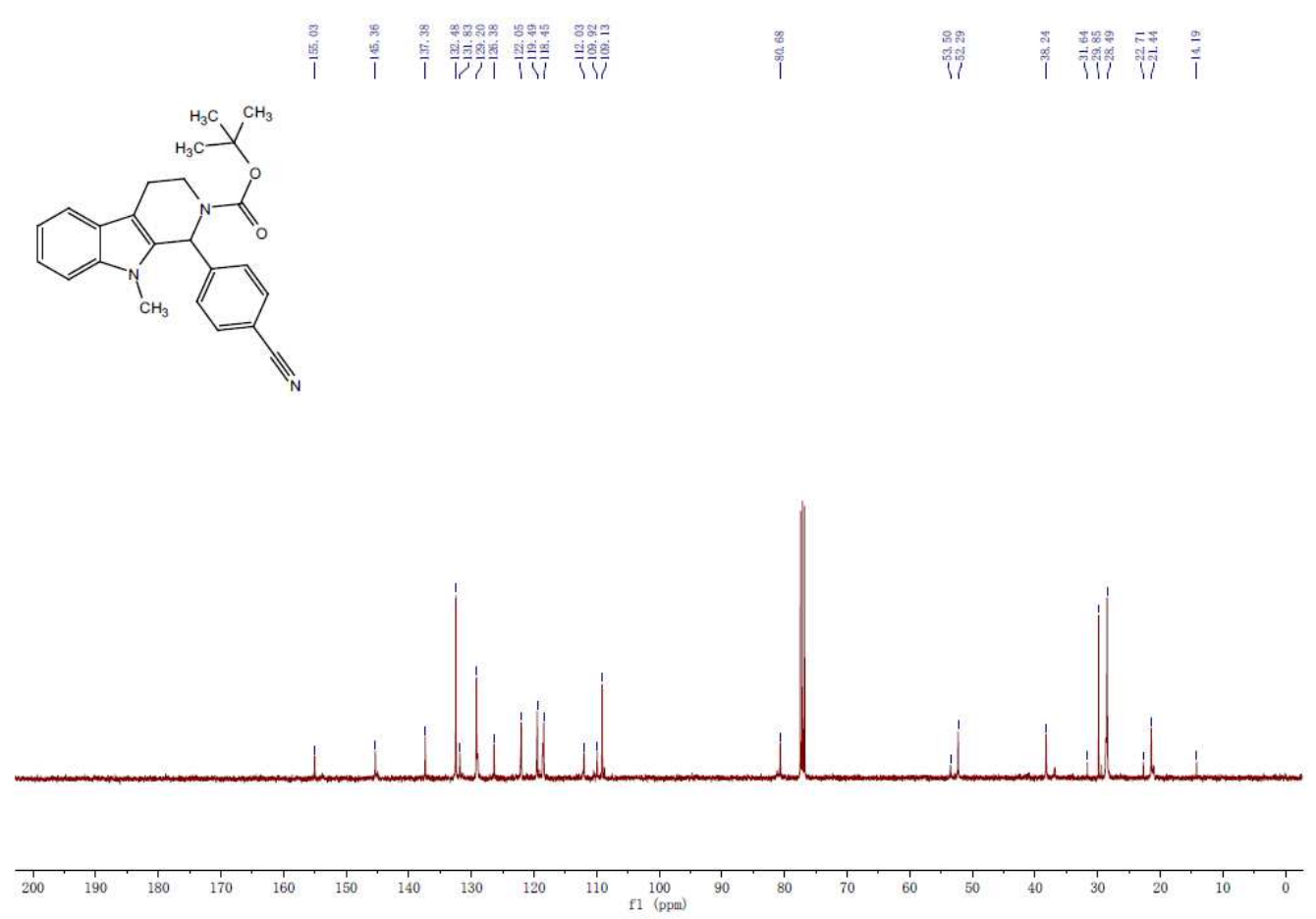
${ }^{1} \mathrm{H}$ NMR spectrum of compound $\mathbf{3 h b}$.

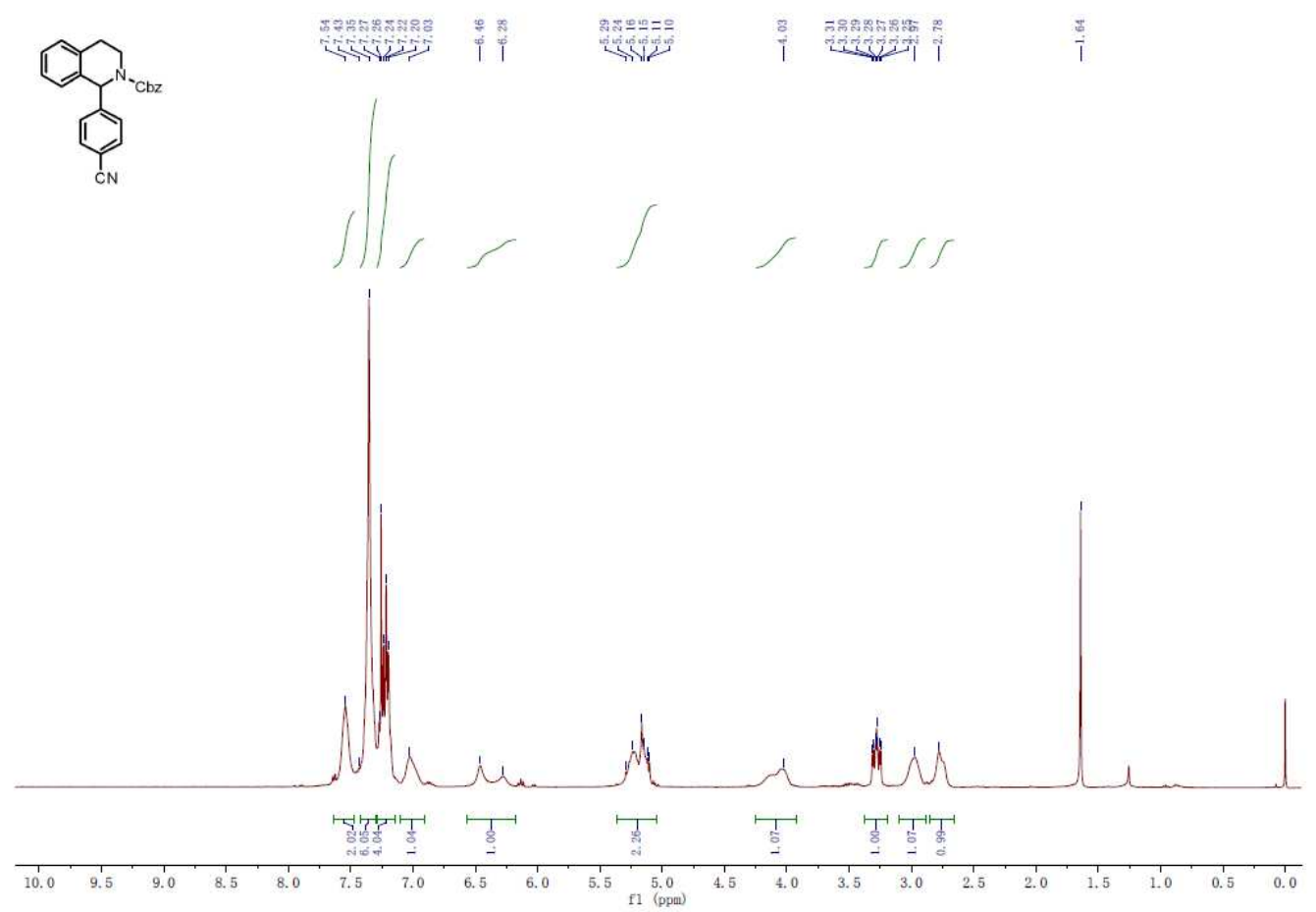

${ }^{13} \mathrm{C}$ NMR spectrum of compound $\mathbf{3 h b}$.
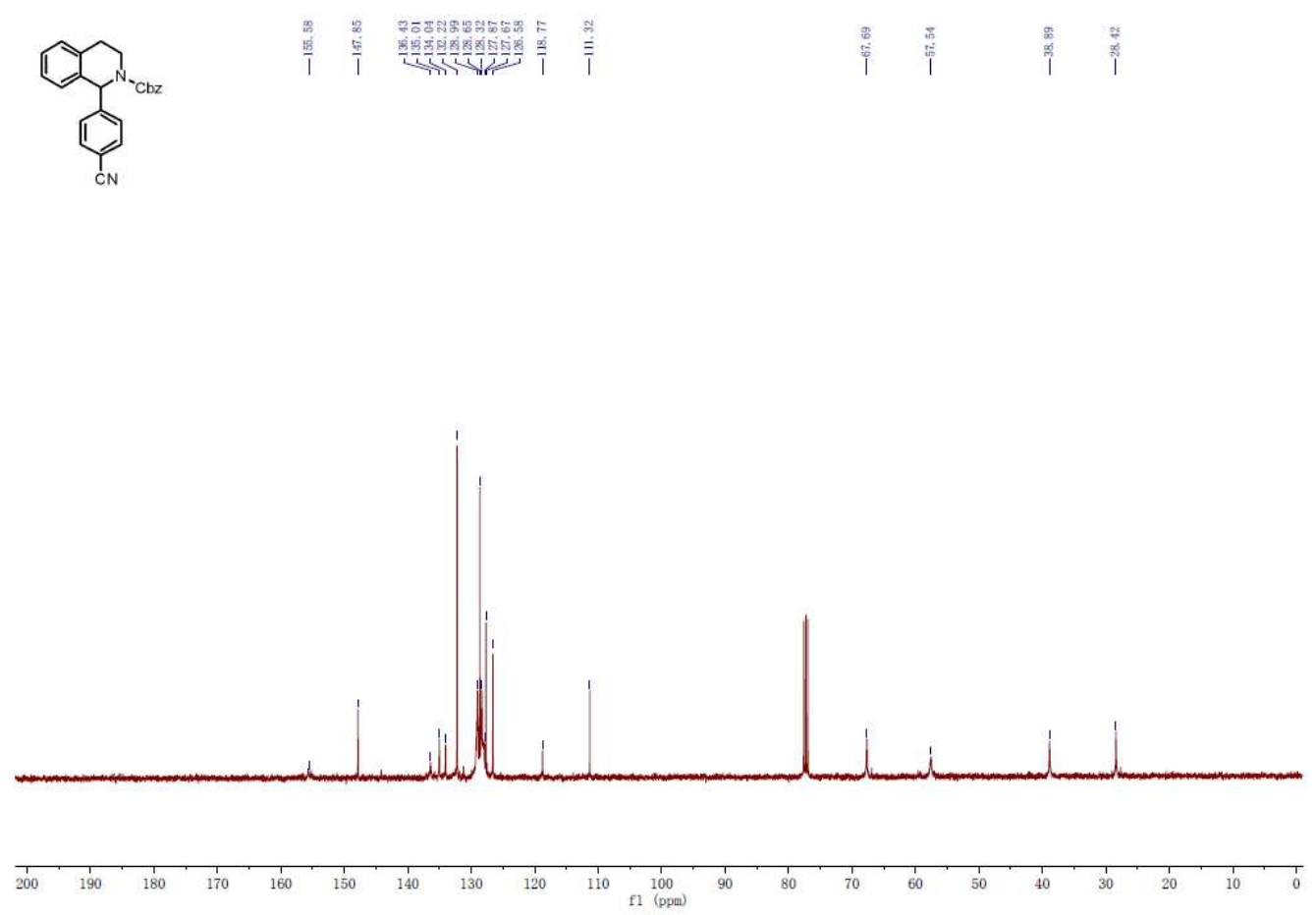
${ }^{1}$ H NMR spectrum of compound 3ib.
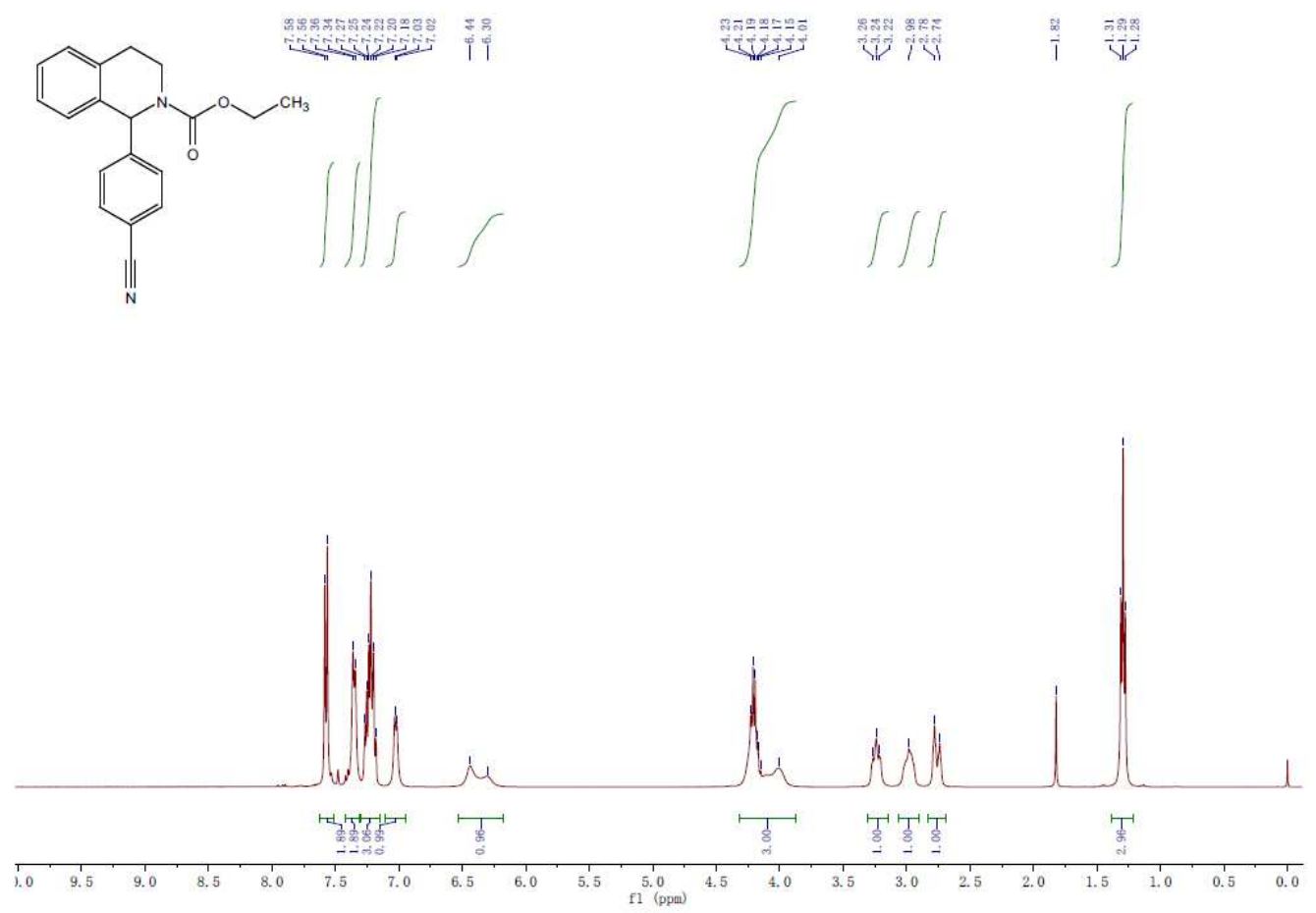

\section{${ }^{13} \mathrm{C}$ NMR spectrum of compound $3 \mathbf{i b}$.}
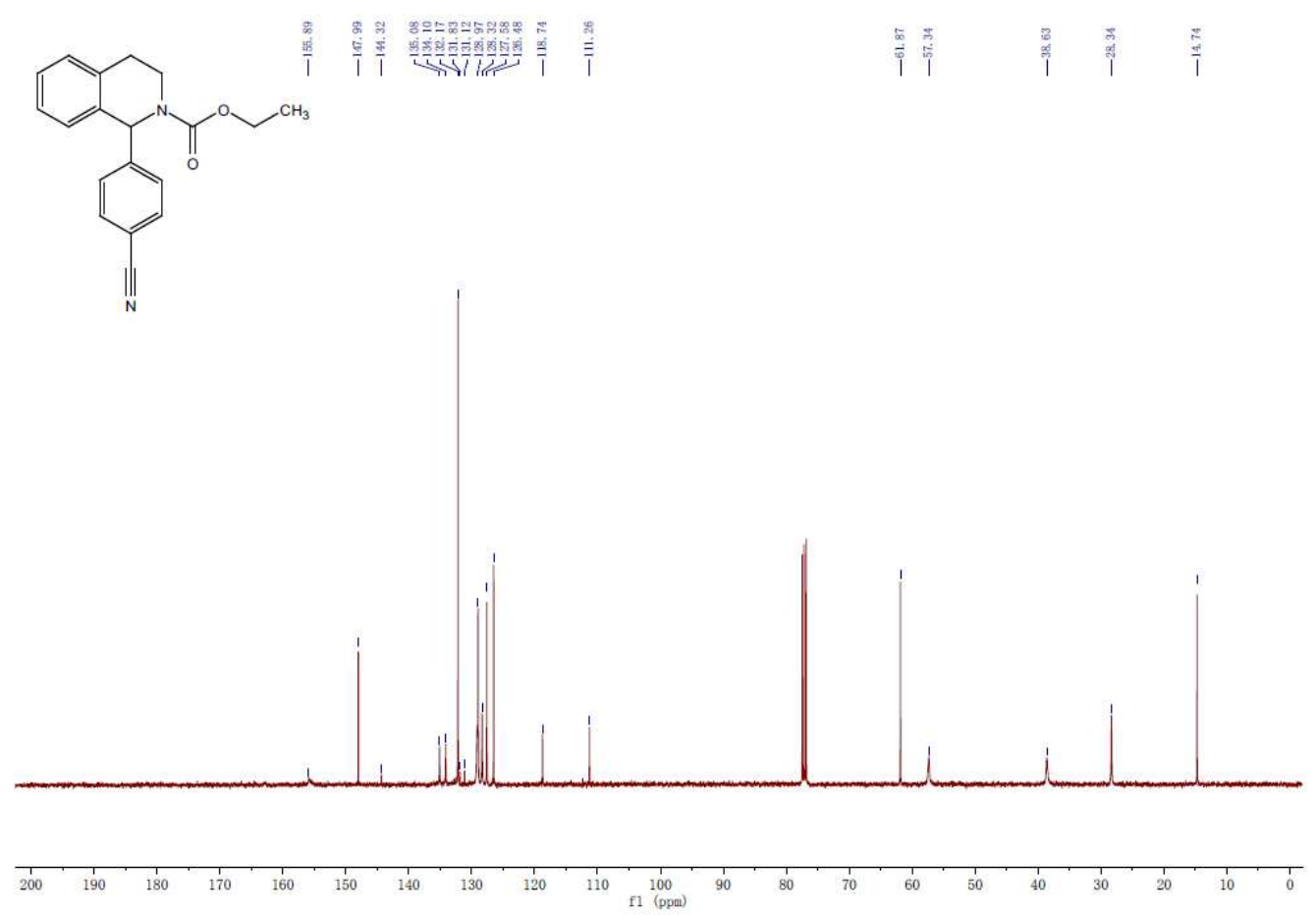
${ }^{1}$ H NMR spectrum of compound $\mathbf{3 j} \mathbf{j b}$.

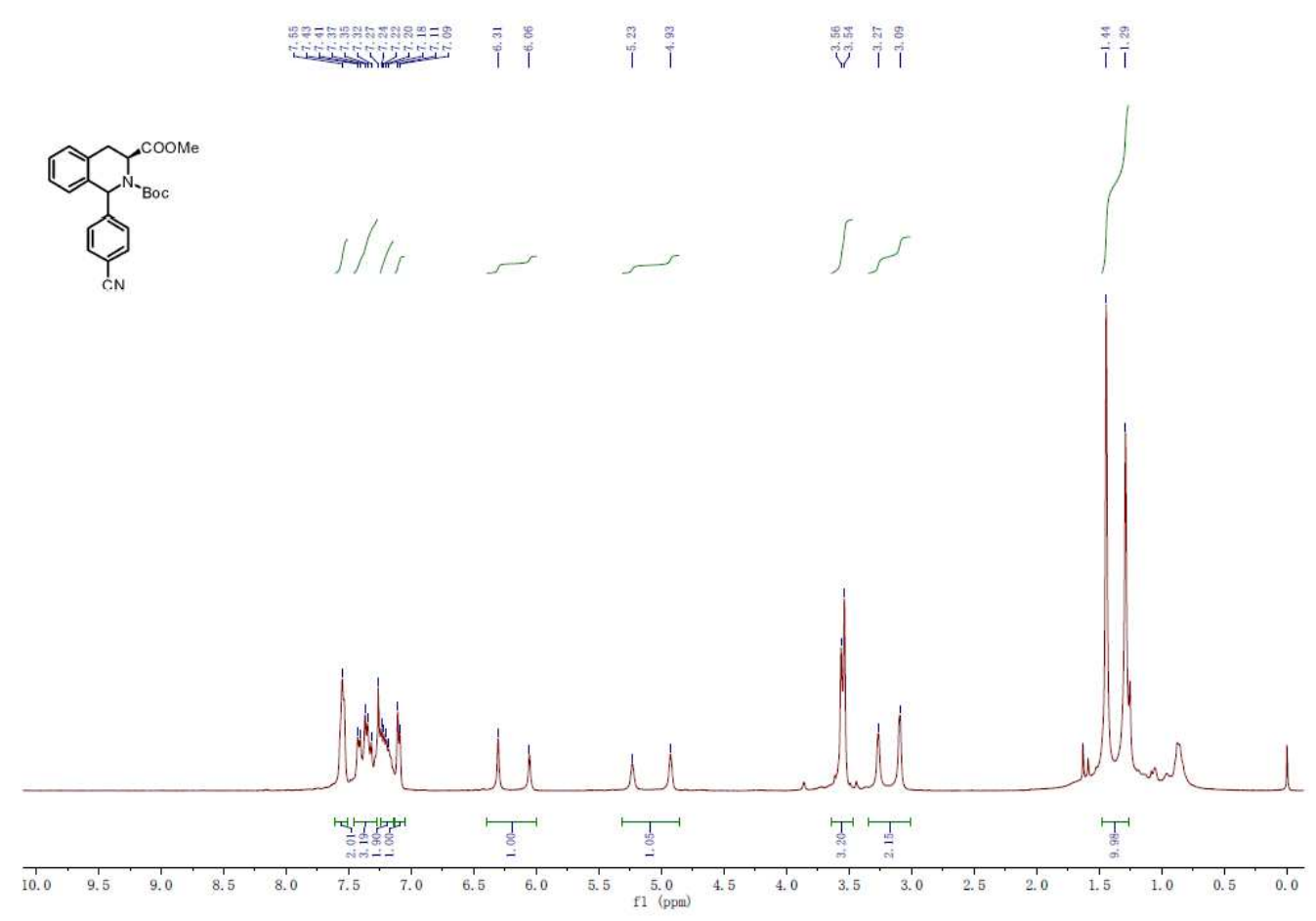

${ }^{13} \mathrm{C}$ NMR spectrum of compound $\mathbf{3 j b}$.
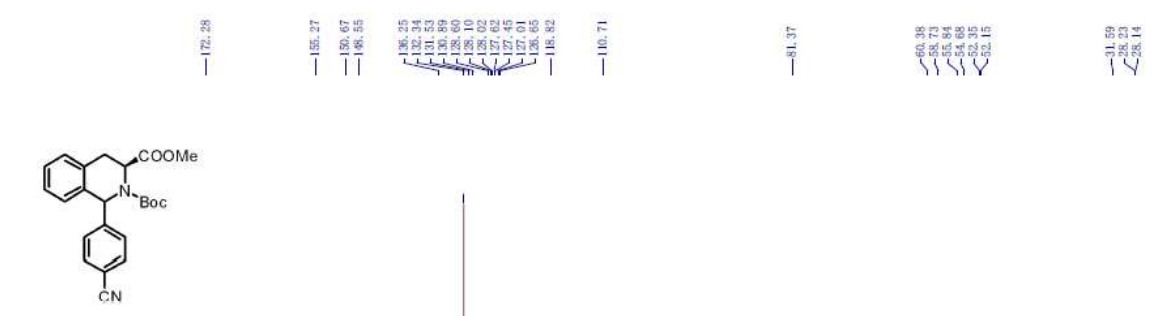
${ }^{1} \mathrm{H}$ NMR spectrum of compound $\mathbf{3 k b}$.

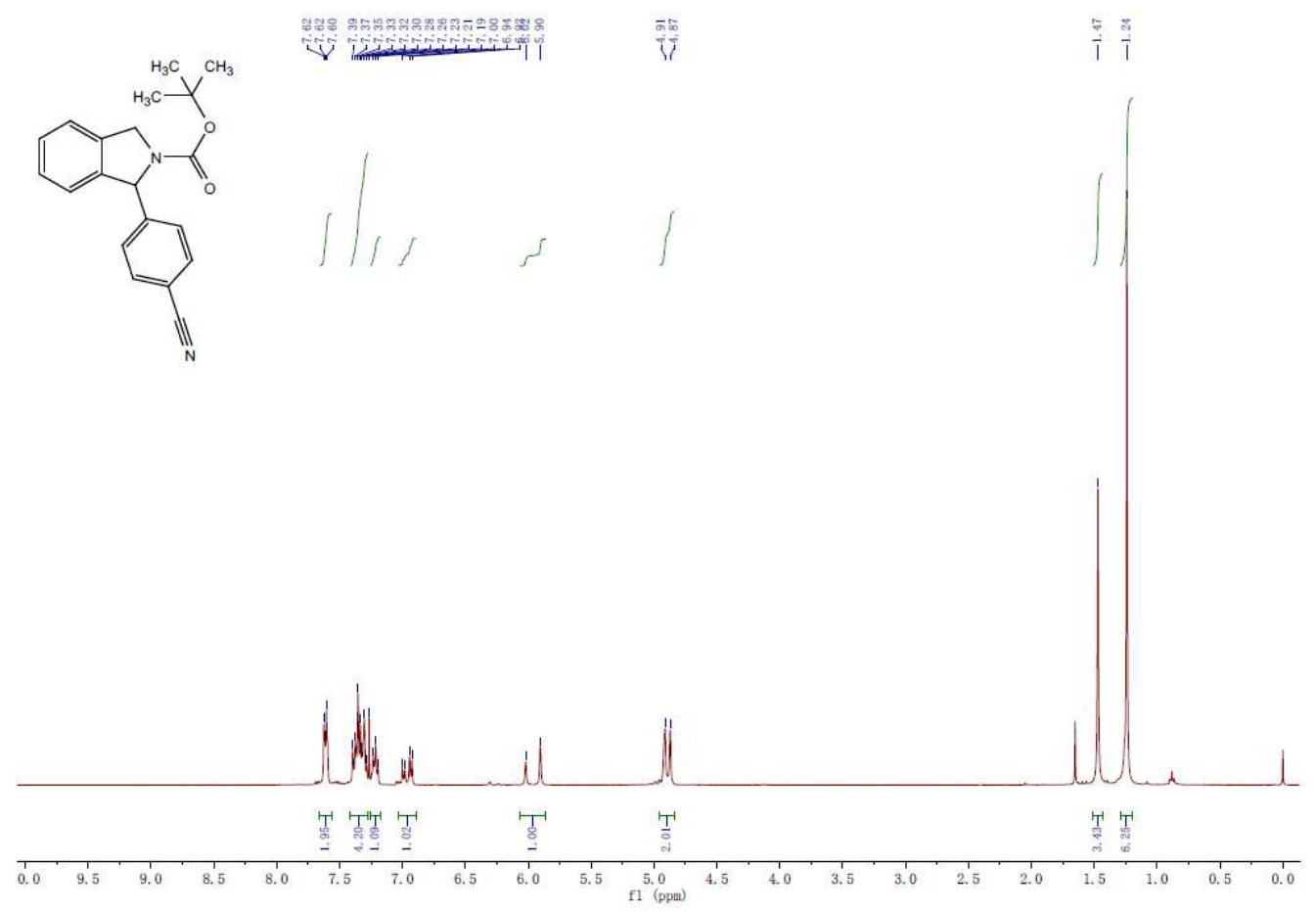

${ }^{13} \mathrm{C}$ NMR spectrum of compound $\mathbf{3 k b}$.

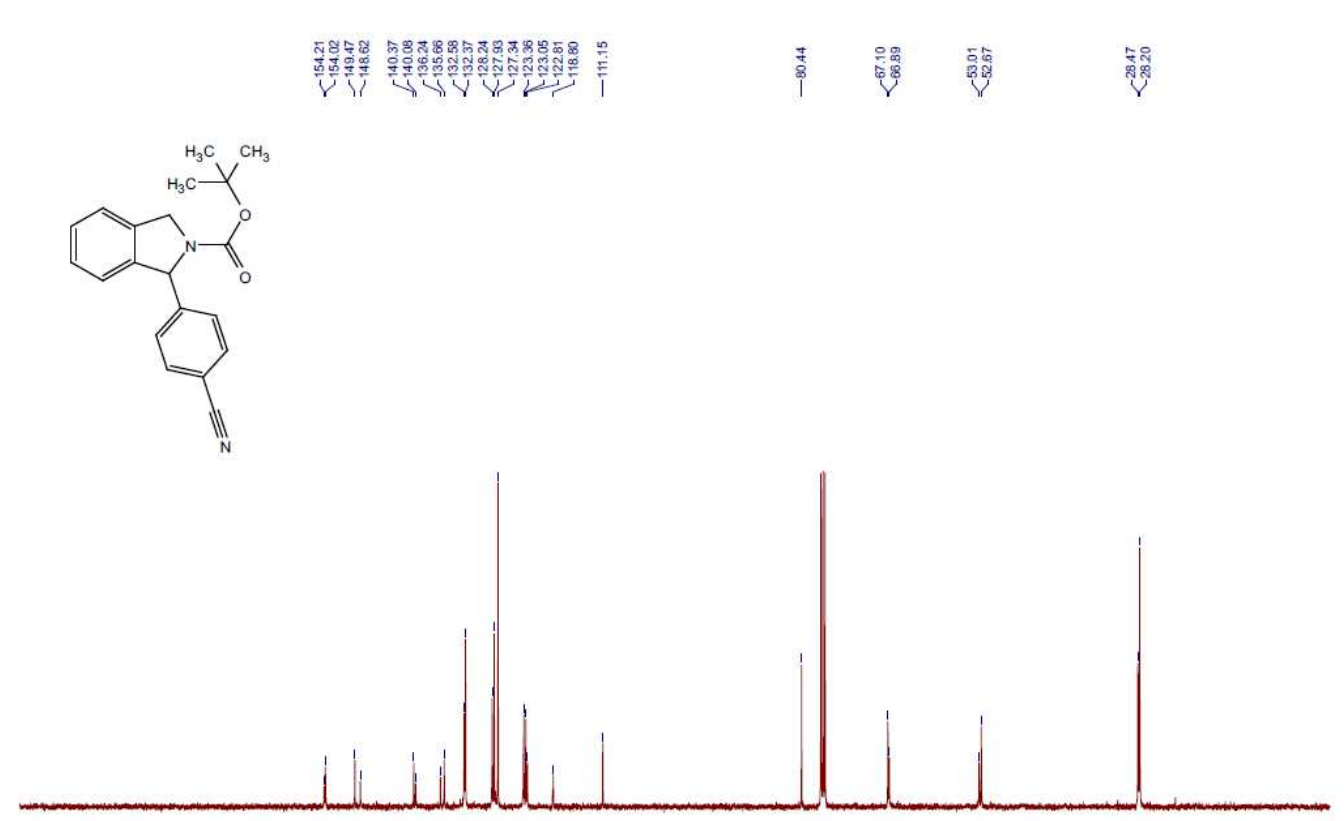

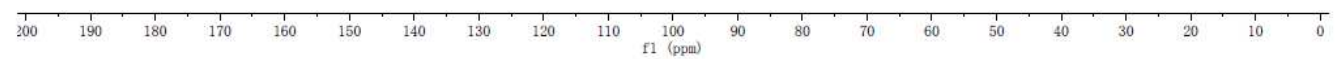


${ }^{1} \mathrm{H}$ NMR spectrum of compound 4.
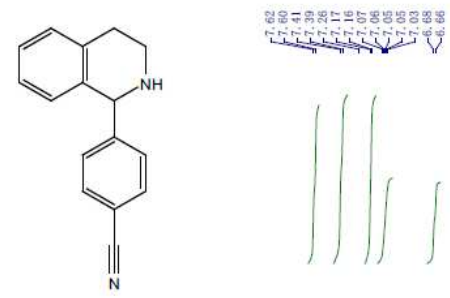

whthy

$\frac{20}{10}$

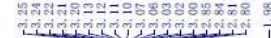
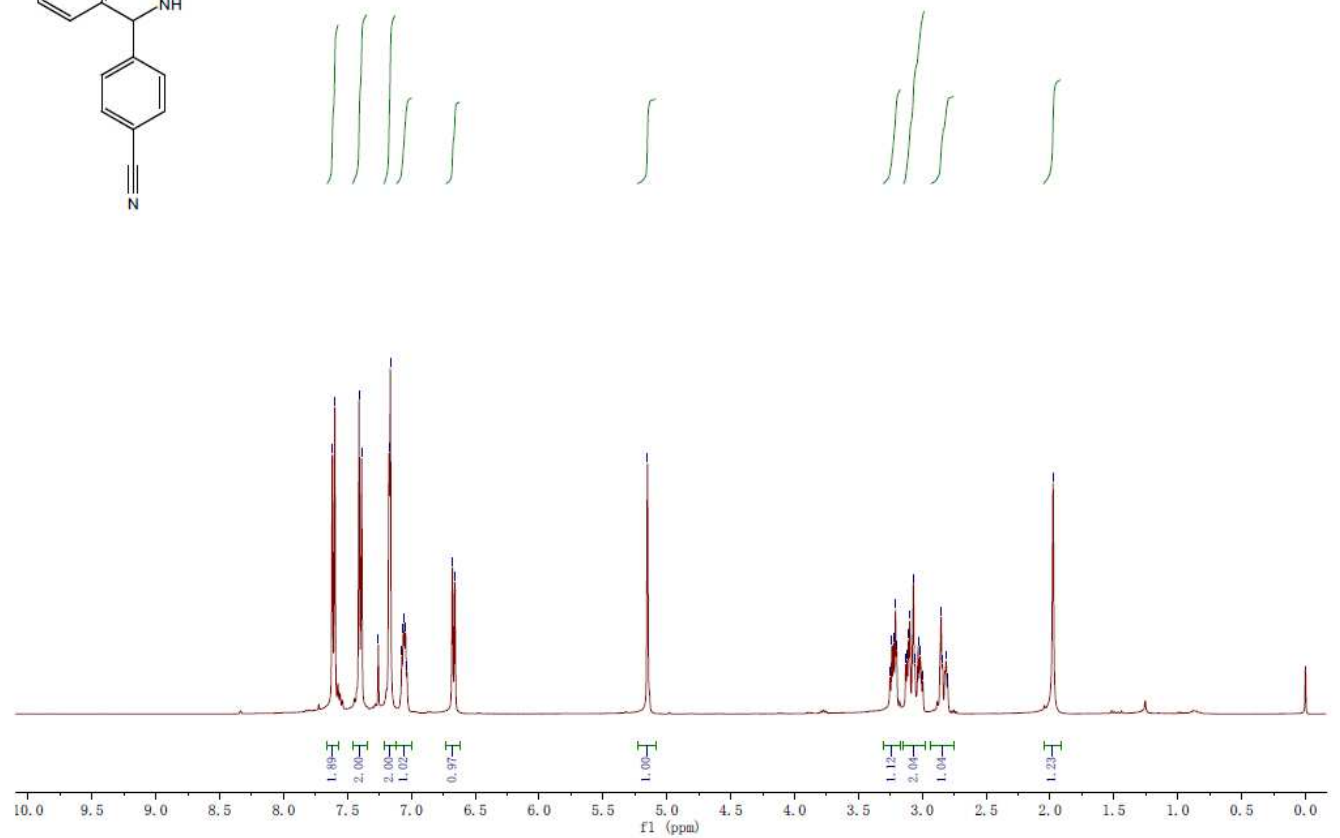

${ }^{13} \mathrm{C}$ NMR spectrum of compound 4.

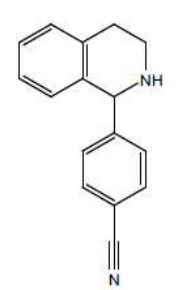

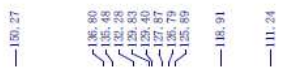
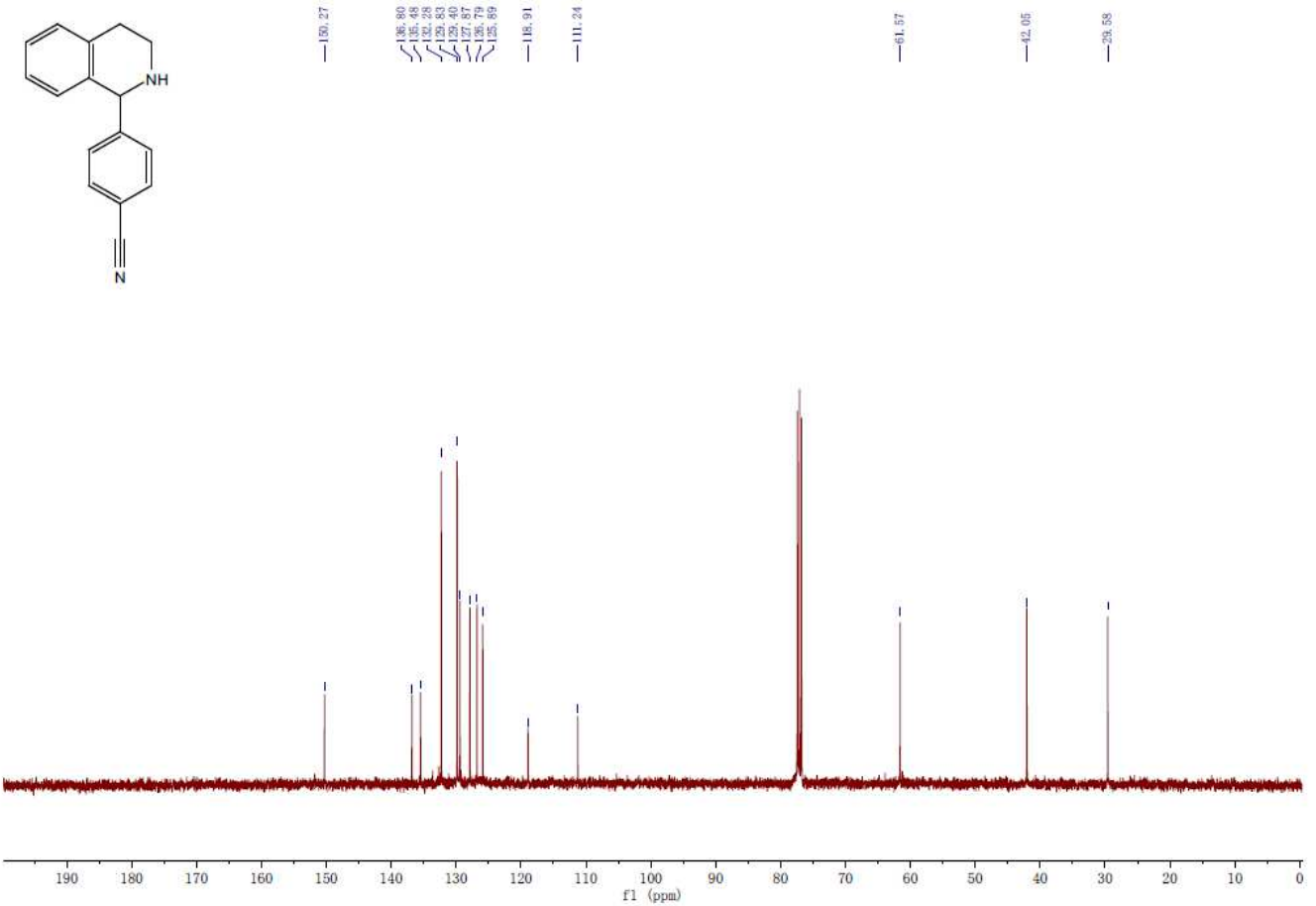